\title{
Long heterochromatic paths in edge-colored graphs
}

\author{
He Chen and Xueliang Li \\ Center for Combinatorics and LPMC \\ Nankai University \\ Tianjin 300071, P.R. China \\ lxl@nankai.edu.cn
}

Submitted: Jan 12, 2005; Accepted: Jun 10, 2005; Published: Jul 29, 2005

AMS Subject Classification (2000): 05C38, 05C15

\begin{abstract}
Let $G$ be an edge-colored graph. A heterochromatic path of $G$ is such a path in which no two edges have the same color. $d^{c}(v)$ denotes the color degree of a vertex $v$ of $G$. In a previous paper, we showed that if $d^{c}(v) \geq k$ for every vertex $v$ of $G$, then $G$ has a heterochromatic path of length at least $\left\lceil\frac{k+1}{2}\right\rceil$. It is easy to see that if $k=1,2, G$ has a heterochromatic path of length at least $k$. Saito conjectured that under the color degree condition $G$ has a heterochromatic path of length at least $\left\lceil\frac{2 k+1}{3}\right\rceil$. Even if this is true, no one knows if it is a best possible lower bound. Although we cannot prove Saito's conjecture, we can show in this paper that if $3 \leq k \leq 7, G$ has a heterochromatic path of length at least $k-1$, and if $k \geq 8, G$ has a heterochromatic path of length at least $\left\lceil\frac{3 k}{5}\right\rceil+1$. Actually, we can show that for $1 \leq k \leq 5$ any graph $G$ under the color degree condition has a heterochromatic path of length at least $k$, with only one exceptional graph $K_{4}$ for $k=3$, one exceptional graph for $k=4$ and three exceptional graphs for $k=5$, for which $G$ has a heterochromatic path of length at least $k-1$. Our experience suggests us to conjecture that under the color degree condition $G$ has a heterochromatic path of length at least $k-1$.
\end{abstract}

\section{Introduction}

We use Bondy and Murty [3] for terminology and notations not defined here and consider simple graphs only.

Let $G=(V, E)$ be a graph. By an edge-coloring of $G$ we will mean a function $C: E \rightarrow$ $\mathbb{N}$, the set of nonnegative integers. If $G$ is assigned such a coloring, then we say that $G$ is an edge-colored graph. Denote the colored graph by $(G, C)$, and call $C(e)$ the color of the 
edge $e \in E$ and $C(u v)=\emptyset$ if $u v \notin E(G)$ for any $u, v \in V(G)$. All edges with the same color form a color class of the graph. For a subgraph $H$ of $G$, we let $C(H)=\{C(e) \mid e \in E(H)\}$ and $c(H)=|C(H)|$. For a vertex $v$ of $G$, the color neighborhood $C N(v)$ of $v$ is defined as the set $\{C(e) \mid e$ is incident with $v\}$ and the color degree is $d^{c}(v)=|C N(v)|$. A path is called heterochromatic if any two edges of it have different colors. If $u$ and $v$ are two vertices on a path $P, u P v$ denotes the segment of $P$ from $u$ to $v$.

There are many existing literature dealing with the existence of paths and cycles with special properties in edge-colored graphs. In [5], the authors showed that for a 2-edgecolored graph $G$ and three specified vertices $x, y$ and $z$, to decide whether there exists a color-alternating path from $x$ to $y$ passing through $z$ is NP-complete. The heterochromatic Hamiltonian cycle or path problem was studied by Hahn and Thomassen [9], Rödl and Winkler (see [8]), Frieze and Reed [8], and Albert, Frieze, Reed [1]. For more references, see $[2,6,7,10,11]$. Many results in these papers are proved by using probabilistic methods.

In [4], the authors showed that if $G$ is an edge-colored graph with $d^{c}(v) \geq k$ for every $v$ of $G$, then $G$ has a heterochromatic path with length at least $\left\lceil\frac{k+1}{2}\right\rceil$. It is easy to see that if $k=1,2, G$ has a heterochromatic path of length at least $k$. Saito conjectured that under the color degree condition $G$ has a heterochromatic path of length at least $\left\lceil\frac{2 k+1}{3}\right\rceil$. Even if this is true, no one knows if it is a best possible lower bound in general. Although we cannot prove Saito's conjecture, we can show in this paper that if $3 \leq k \leq 7$, then $G$ has a heterochromatic path of length at least $k-1$, and if $k \geq 8$, then $G$ has a heterochromatic path of length at least $\left\lceil\frac{3 k}{5}\right\rceil+1$. Actually, we can show that for $1 \leq k \leq 5$ any graph $G$ under the color degree condition has a heterochromatic path of length at least $k$, with only one exceptional graph $K_{4}$ for $k=3$, one exceptional graphs for $k=4$ and three exceptional graphs for $k=5$, for which $G$ has a heterochromatic path of length at least $k-1$. Our experience suggests us to conjecture that under the color degree condition $G$ has a heterochromatic path of length at least $k-1$.

\section{Long heterochromatic paths for $k \leq 7$}

We consider the case when $1 \leq k \leq 7$, first.

For the case when $k=1$ or 2 , it is obvious that there is a heterochromatic path of length $k$ in $G$. In fact, for $k=1$ any edge of $G$ is a required heterochromatic path of length $k$; for $k=2$, at each vertex there exist two adjacent edges with different colors, and they form a required heterochromatic path of length $k$. Next, we consider the case when $3 \leq k \leq 7$ and get the following result.

Theorem 2.1 Let $G$ be an edge-colored graph and $3 \leq k \leq 7$ an integer. Suppose that $d^{c}(v) \geq k$ for every vertex $v$ of $G$. Then $G$ has a heterochromatic path of length at least $k-1$.

Proof. (1) $k=3$. Since $k=3>2$, there is a heterochromatic path of length 2 in $G$. 
(2) $k=4$. Since $k=4>3$, there is a heterochromatic path of length 2 in $G$. Let $P=u_{1} u_{2} u_{3}$ be such a path that $u_{1} u_{2}$ has color $i_{1}$ and $u_{2} u_{3}$ has color $i_{2}$ with $i_{1} \neq i_{2}$. Since $d^{c}\left(u_{3}\right) \geq k=4$, there are two vertices $v, w \in G$ such that $u_{3} v, u_{3} w \in E(G)$ have two different colors $i_{3}, i_{4} \notin\left\{i_{1}, i_{2}\right\}$. Let $u_{4}$ be a vertex in $\{v, w\} \backslash\left\{u_{1}\right\}$. Then $\left\{u_{4}\right\} \cap\left\{u_{1}, u_{2}, u_{3}\right\}=\emptyset$ and $P^{\prime}=u_{1} u_{2} u_{3} u_{4}$ is a heterochromatic path of length 3 .

(3) $k=5$. Since $k=5>4$, there is a heterochromatic path of length 3 in $G$. Let $P=u_{1} u_{2} u_{3} u_{4}$ be such a path that $u_{x} u_{x+1}$ has color $i_{x}$ for $x=1,2,3$. If there exists a $v \notin\left\{u_{1}, u_{2}, u_{3}, u_{4}\right\}$ such that $C\left(u_{4} v\right) \notin\left\{i_{1}, i_{2}, i_{3}\right\}$, then $P^{\prime}=u_{1} u_{2} u_{3} u_{4} v$ is a heterochromatic path of length 4 . Otherwise, since $d^{c}\left(u_{4}\right) \geq 5$, we have that $\left|C\left(\left\{u_{1} u_{4}, u_{2} u_{4}\right\}\right)-\left\{i_{1}, i_{2}, i_{3}\right\}\right|=2$ and there exists a $v_{1} \in V(G)$ such that $C\left(u_{4} v_{1}\right)=i_{1}$. Since $d^{c}\left(v_{1}\right) \geq 5$, we have that $\left|C N\left(v_{1}\right)-\left\{i_{1}, i_{2}, i_{3}\right\}\right| \geq 2$. If there exists a $v_{2} \in V(G)$ such that $C\left(v_{1} v_{2}\right) \notin\left\{i_{1}, i_{2}, i_{3}\right\}$, then $P^{\prime}=u_{2} u_{3} u_{4} v_{1} v_{2}$ is a heterochromatic path of length 4; if $C\left(u_{1} v_{1}\right) \notin\left\{i_{1}, i_{2}, i_{3}\right\}$, then $P^{\prime}=v_{1} u_{1} u_{2} u_{3} u_{4}$ is a heterochromatic path of length 4 . So, the only remaining case is when $\left|C\left(\left\{u_{2} v_{1}, u_{3} v_{1}\right\}\right)-\left\{i_{1}, i_{2}, i_{3}\right\}\right|=2$, and in this case we have that $P^{\prime}=u_{1} u_{2} v_{1} u_{3} u_{4}$ is a heterochromatic path of length 4 .

(4) $k=6$. Since $k=6>5$, there is a heterochromatic path of length 4 in $G$. Let $P=u_{1} u_{2} u_{3} u_{4} u_{5}$ be such a path that $u_{x} u_{x+1}$ has color $i_{x}$ for $x=1,2,3,4$. If there exists a $v \notin\left\{u_{1}, u_{2}, u_{3}, u_{4}, u_{5}\right\}$ such that $C\left(u_{5} v\right) \notin\left\{i_{1}, \ldots, i_{4}\right\}$, then $u_{1} u_{2} u_{3} u_{4} u_{5} v$ is a heterochromatic path of length 5. Next we consider the case when there is no such a vertex $v$, in other words, $\left|C\left(\left\{u_{1} u_{5}, u_{2} u_{5}, u_{3} u_{5}\right\}\right)-\left\{i_{1}, i_{2}, i_{3}, i_{4}\right\}\right| \geq 2$. Since $d^{c}\left(u_{5}\right) \geq 6$, there is a vertex $v_{1}$ such that $C\left(u_{5} v_{1}\right)=i_{1}$ or $i_{2}$.

(4.1) $C\left(u_{5} v_{1}\right)=i_{1}$. Since $d^{c}\left(u_{5}\right) \geq 6$ and $\left|C\left(\left\{u_{1} u_{5}, u_{2} u_{5}, u_{3} u_{5}\right\}\right)-\left\{i_{1}, i_{2}, i_{3}, i_{4}\right\}\right| \geq 2$, there exists a $u_{6} \in V(G)$ such that $C\left(u_{5} u_{6}\right)=i_{2}$ or $i_{3}$. If there is a vertex $v \notin$ $\left\{u_{2}, u_{3}, u_{4}, u_{5}\right\}$ such that $C\left(v_{1} v\right) \notin\left\{i_{1}, \ldots, i_{4}\right\}$, then $u_{2} u_{3} u_{4} u_{5} v_{1} v$ is a heterochromatic path of length 5 . And, since $d^{c}\left(v_{1}\right) \geq k=6$, we have that $\mid C\left(\left\{u_{2} v_{1}, u_{3} v_{1}, u_{4} v_{1}\right\}\right)-$ $\left\{i_{1}, \ldots, i_{4}\right\} \mid \geq 2$. If there exists an $2 \leq i \leq 3$ such that $\left|C\left(\left\{u_{i} v_{1}, u_{i+1} v_{1}\right\}\right)-\left\{i_{1}, i_{2}, i_{3}, i_{4}\right\}\right|$ $=2$, then $P^{\prime}=u_{1} P u_{i} v_{1} u_{i+1} P u_{5}$ is a heterochromatic path of length 5 . Otherwise, $\left|C\left(\left\{u_{2} v_{1}, u_{4} v_{1}\right\}\right)-\left\{i_{1}, i_{2}, i_{3}, i_{4}\right\}\right|=2$ and $u_{1} u_{2} v_{1} u_{4} u_{5} u_{6}$ is a heterochromatic path of length 5 .

(4.2) $C\left(u_{5} v_{1}\right)=i_{2}$. Since $d^{c}\left(u_{5}\right) \geq 6$, there exists a $u_{6} \in V(G)$ such that $C\left(u_{5} u_{6}\right)=i_{3}$. Then we have the following three cases: (i) There is no vertex $v \notin\left\{u_{1}, \ldots, u_{5}\right\}$ such that $C\left(v_{1} v\right) \notin\left\{i_{1}, \ldots, i_{4}\right\}$. Since $d^{c}\left(v_{1}\right) \geq 6$, we have that $\mid C\left(\left\{u_{1} v_{1}, u_{2} v_{1}, u_{3} v_{1}, u_{4} v_{1}\right\}\right)-$ $\left\{i_{1}, i_{2}, i_{3}, i_{4}\right\} \mid \geq 2$. If $C\left(u_{1} v_{1}\right) \notin\left\{i_{1}, i_{2}, i_{3}, i_{4}\right\}$, then $v_{1} u_{1} u_{2} u_{3} u_{4} u_{5}$ is a heterochromatic path of length 5 . If there exists an $i=2$ or 3 such that $\left|C\left(\left\{u_{i} v_{1}, u_{i+1} v_{1}\right\}\right)-\left\{i_{1}, i_{2}, i_{3}, i_{4}\right\}\right|=2$, then $u_{1} P u_{i} v_{1} u_{i+1} P u_{5}$ is a heterochromatic path of length 5. Otherwise, $\mid C\left(\left\{u_{2} v_{1}, u_{4} v_{1}\right\}\right)-$ $\left\{i_{1}, i_{2}, i_{3}, i_{4}\right\} \mid=2$, and then $u_{1} u_{2} v_{1} u_{4} u_{5} u_{6}$ is a heterochromatic path of length 5 . (ii) There is a $v_{2} \notin\left\{u_{1}, \ldots, u_{5}\right\}$ such that $C\left(v_{1} v_{2}\right)-\left\{i_{1}, \ldots, i_{4}\right\} \neq \emptyset$ and there is no vertex $v \notin\left\{u_{1}, \ldots, u_{5}\right\}$ such that $\left|C\left(\left\{v_{1} v_{2}, v_{2} v\right\}\right)-\left\{i_{1}, \ldots, i_{4}\right\}\right|=2$. Let $i_{5}=C\left(v_{1} v_{2}\right)$. Since $d^{c}\left(v_{2}\right) \geq 6$, we have that $C\left(\left\{u_{1} v_{2}, u_{2} v_{2}, u_{3} v_{2}, u_{4} v_{2}\right\}\right)-\left\{i_{1}, \ldots, i_{5}\right\} \neq \emptyset$. If $C\left(u_{i} v_{2}\right)-$ $\left\{i_{1}, \ldots, i_{5}\right\} \neq \emptyset$ for $i=1$ or 2 , then $u_{3} u_{4} u_{5} v_{1} v_{2} u_{i}$ is a heterochromatic path of length 5. If $C\left(u_{4} v_{2}\right)-\left\{i_{1}, \ldots, i_{5}\right\} \neq \emptyset$, then $u_{1} u_{2} u_{3} u_{4} v_{2} v_{1}$ is a heterochromatic path of length 5. Otherwise, $C\left(u_{3} v_{2}\right)-\left\{i_{1}, \ldots, i_{5}\right\} \neq \emptyset$. Since $u_{1} u_{2} u_{3} u_{4} u_{5}$ is a heterochromatic path of length 4 , we have that $\left|C\left(\left\{u_{1} u_{3}, u_{1} u_{4}, u_{1} u_{5}\right\}\right)-\left\{i_{1}, i_{2}, i_{3}, i_{4}\right\}\right| \geq 2$ which implies that 
$C\left(\left\{u_{1} u_{3}, u_{1} u_{4}\right\}\right)-\left\{i_{1}, i_{2}, i_{3}, i_{4}\right\} \neq \emptyset$. If $C\left(u_{1} u_{3}\right)-\left\{i_{1}, i_{2}, i_{3}, i_{4}\right\} \neq \emptyset$, then $u_{2} u_{1} u_{3} u_{4} u_{5} v_{1}$ is a heterochromatic path of length 5 . If $C\left(u_{1} u_{4}\right) \notin\left\{i_{1}, i_{2}, i_{3}, i_{4}\right\}$, then $u_{5} u_{4} u_{1} u_{2} u_{3} v_{2}$ or $u_{2} u_{1} u_{4} u_{5} v_{1} v_{2}$ is a heterochromatic path of length 5. (iii) There exist $v_{2}, v_{3} \notin\left\{u_{1}, \ldots, u_{5}\right\}$ such that $\left|C\left(\left\{v_{1} v_{2}, v_{2} v_{3}\right\}\right)-\left\{i_{1}, \ldots, i_{4}\right\}\right|=2$. Then $u_{3} u_{4} u_{5} v_{1} v_{2} v_{3}$ is a heterochromatic path of length 5 .

(5) $k=7$. Since $k=7>6$, there is a heterochromatic path of length 5 in $G$. Let $P=u_{1} u_{2} u_{3} u_{4} u_{5} u_{6}$ be such a path that $u_{x} u_{x+1}$ has color $i_{x}$ for $x=1, \ldots, 5$. If there is a vertex $v \notin V(P)$ such that $C\left(u_{6} v\right) \notin C(P)$, then $u_{1} P u_{6} v$ is a heterochromatic path of length 6 . Next we consider the case when there is no such $v$. Since $d^{c}\left(u_{6}\right) \geq 7$, we have that $\left|C\left(\left\{u_{1} u_{6}, u_{2} u_{6}, u_{3} u_{6}, u_{4} u_{6}\right\}\right)-\left\{i_{1}, \ldots, i_{5}\right\}\right| \geq 2$. Let $k_{0}=\min \{k \mid$ there is a vertex $v \notin V(P)$ such that $\left.C\left(u_{6} v\right)=i_{k}\right\}$, and $v_{1}$ be a vertex $\notin V(P)$ such that $C\left(u_{6} v\right)=i_{k_{0}}$. Then $k_{0}=1$ or 2 or 3 .

(5.1) $k_{0}=1$. If there exists a $v_{2} \notin V(P)$ such that $C\left(v_{1} v_{2}\right) \notin C(P)$, then $u_{2} P u_{6} v_{1} v_{2}$ is a heterochromatic path of length 6 . Or, $\left|C\left(\left\{u_{1} v_{1}, \ldots, u_{5} v_{1}\right\}\right)-C(P)\right| \geq 2$. If there is a vertex $u \notin V(P)$ such that $C\left(u u_{1}\right) \notin C(P)$, then $u u_{1} P u_{6}$ is a heterochromatic path of length 6. Next we consider the case when $\left|C\left(\left\{u_{2} v_{1}, u_{3} v_{1}, u_{4} v_{1}, u_{5} v_{1}\right\}\right)-C(P)\right| \geq 2$ and $\left|C\left(\left\{u_{1} u_{3}, \ldots, u_{1} u_{6}\right\}\right)-C(P)\right| \geq 2$. If $C\left(u_{2} v_{1}\right) \notin C(P)$, then there is an $3 \leq i \leq 6$ such that $C\left(u_{1} u_{i}\right) \notin C(P) \cup C\left(u_{2} v_{1}\right)$, and so $u_{i-1} P^{-1} u_{2} v_{1} u_{6} P^{-1} u_{i} u_{1}$ is a heterochromatic path of length 6 . If there exists an $i=3$ or 4 such that $\mid C\left(\left\{u_{i} v_{1}, u_{i+1} v_{1}\right\}\right)-$ $C(P) \mid=2$, then $u_{1} P u_{i} v_{1} u_{i+1} P u_{6}$ is a heterochromatic path of length 6 . In the rest we shall only consider the case when $\left|C\left(\left\{u_{3} v_{1}, u_{5} v_{1}\right\}\right)-C(P)\right|=2$, and let $i_{6}=C\left(u_{3} v_{1}\right)$ and $i_{7}=C\left(u_{5} v_{1}\right)$. If there exists a $v \notin V(P)$ such that $C\left(u_{6} v\right) \notin\left\{i_{1}, i_{2}, i_{5}\right\}$, i.e., $C\left(u_{6} v\right) \in\left\{i_{3}, i_{4}\right\}$, then $u_{1} u_{2} u_{3} v_{1} u_{5} u_{6} v$ is a heterochromatic path of length 6 . Otherwise, $\left|C\left(\left\{u_{1} u_{6}, u_{2} u_{6}, u_{3} u_{6}, u_{4} u_{6}\right\}\right)-\left\{i_{1}, i_{2}, i_{5}\right\}\right|=4$. On the other hand, if $C\left(u_{1} u_{6}\right)-\left\{i_{1}, i_{2}, i_{5}\right\}$ $\neq \emptyset$, then $v_{1} u_{3} u_{2} u_{1} u_{6} u_{5} u_{4}$ or $v_{1} u_{5} u_{6} u_{1} u_{2} u_{3} u_{4}$ is a heterochromatic path of length 6 , a contradiction.

(5.2) $k_{0}=2$. So, $\left|C\left(\left\{u_{1} u_{6}, u_{2} u_{6}, u_{3} u_{6}, u_{4} u_{6}\right\}\right)-\left\{i_{2}, i_{3}, i_{4}, i_{5}\right\}\right| \geq 3$. We have the following three cases:

(i) There is no vertex $v \notin V(P)$ such that $C\left(v_{1} v\right) \notin C(P)$. Since $d^{c}\left(v_{1}\right) \geq 7$, we have that $\left|C\left(\left\{u_{1} v_{1}, u_{2} v_{1}, u_{3} v_{1}, u_{4} v_{1}, u_{5} v_{1}\right\}\right)-C(P)\right| \geq 2$. If there exists a $u \notin V(P)$ such that $C\left(u u_{1}\right) \notin C(P)$, then $u u_{1} P u_{6}$ is a heterochromatic path of length 6 . Next we consider the case when $\left|C\left(\left\{u_{2} v_{1}, u_{3} v_{1}, u_{4} v_{1}, u_{5} v_{1}\right\}\right)-C(P)\right| \geq 2$ and $\left|C\left(\left\{u_{1} u_{3}, \ldots, u_{1} u_{6}\right\}\right)-C(P)\right| \geq 2$. If $C\left(u_{2} v_{1}\right) \notin C(P)$, then there is an $3 \leq i \leq 6$ such that $C\left(u_{1} u_{i}\right) \notin C(P) \cup C\left(u_{2} v_{1}\right)$, and so $u_{3} P u_{i} u_{1} u_{2} v_{1} u_{6} P^{-1} u_{i+1}$ is a heterochromatic path of length 6 . If there exists an $i=3$ or 4 such that $\left|C\left(\left\{u_{i} v_{1}, u_{i+1} v_{1}\right\}\right)-C(P)\right|=2$, then $u_{1} P u_{i} v_{1} u_{i+1} P u_{6}$ is a heterochromatic path of length 6 . In the rest we shall only consider the case when $\left|C\left(\left\{u_{3} v_{1}, u_{5} v_{1}\right\}\right)-C(P)\right|=2$. Since $\left|C\left(\left\{u_{1} u_{6}, u_{2} u_{6}, u_{3} u_{6}, u_{4} u_{6}\right\}\right)-\left\{i_{2}, i_{3}, i_{4}, i_{5}\right\}\right| \geq 3$, there exists a $v \notin V(P)$ such that $C\left(u_{6} v\right) \in\left\{i_{3}, i_{4}\right\}$, and so $u_{1} u_{2} u_{3} v_{1} u_{5} u_{6} v$ is a heterochromatic path of length 6 .

(ii) There exists a $v_{2} \notin V(P)$ such that $C\left(v_{1} v_{2}\right) \notin C(P)$, and there is no $v \notin V(P) \cup$ $\left\{v_{1}\right\}$ such that $C\left(v_{2} v\right) \notin C(P) \cup C\left(v_{1} v_{2}\right)$. Let $i_{6}=C\left(v_{1} v_{2}\right)$. Then $C\left(\left\{u_{1} v_{2}, \ldots, u_{5} v_{2}\right\}\right)-$ $\left\{i_{1}, \ldots, i_{6}\right\} \neq \emptyset$. If there exists a $u \notin\left\{u_{1}, \ldots, u_{6}\right\}$ such that $C\left(u u_{1}\right) \notin C(P)$, then $u u_{1} P u_{6}$ is a heterochromatic path of length 6 . If $C\left(u_{2} v_{2}\right) \notin\left\{i_{1}, \ldots, i_{6}\right\}$, then $v_{1} v_{2} u_{2} P u_{6}$ is a heterochromatic path of length 6 . If $C\left(u_{5} v_{2}\right) \notin\left\{i_{1}, \ldots, i_{6}\right\}$, then $u_{1} P u_{5} v_{2} v_{1}$ is a heterochro- 
matic path of length 6 . So we shall only consider the case when $\mid C\left(\left\{u_{1} u_{3}, \ldots, u_{1} u_{6}\right\}\right)-$ $C(P) \mid \geq 2$ and $C\left(u_{3} v_{2}\right) \notin\left\{i_{1}, \ldots, i_{6}\right\}$ or $C\left(u_{4} v_{2}\right) \notin\left\{i_{1}, \ldots, i_{6}\right\}$.

(ii.1) $C\left(u_{3} v_{2}\right) \notin\left\{i_{1}, \ldots, i_{6}\right\}$, and let $i_{7}=C\left(u_{3} v_{2}\right)$. Since $P$ is a heterochromatic path of length 5 , we have that $C\left(\left\{u_{1} u_{3}, \ldots, u_{1} u_{6}\right\}\right)-\left\{i_{1}, \ldots, i_{5}, i_{7}\right\} \neq \emptyset$. If $C\left(u_{1} u_{3}\right) \notin$ $\left\{i_{1}, \ldots, i_{5}, i_{7}\right\}$, let $P^{\prime}=u_{2} u_{1} u_{3} P u_{6} v_{1}$; if $C\left(u_{1} u_{4}\right) \notin\left\{i_{1}, \ldots, i_{5}, i_{7}\right\}$, let $P^{\prime}=v_{2} u_{3} u_{2} u_{1} u_{4}$ $u_{5} u_{6}$; if $C\left(u_{1} u_{5}\right) \notin\left\{i_{1}, \ldots, i_{5}, i_{6}, i_{7}\right\}$, let $P^{\prime}=v_{1} v_{2} u_{3} u_{2} u_{1} u_{5} u_{6}$; if $C\left(u_{1} u_{6}\right) \notin\left\{i_{1}, \ldots, i_{5}\right.$, $\left.i_{7}\right\}$, let $P^{\prime}=v_{2} u_{3} u_{2} u_{1} u_{6} u_{5} u_{4}$. Then, $P^{\prime}$ is a heterochromatic path of length 6 in all these cases. It remains to show that when $C\left(u_{1} u_{5}\right)=i_{6}$, there is a heterochromatic path of length 6 . Since $d^{c}\left(u_{6}\right) \geq 7$ and $\left|C\left(\left\{u_{1} u_{6}, \ldots, u_{4} u_{6}\right\}\right)-\left\{i_{2}, i_{3}, i_{4}, i_{5}\right\}\right| \geq 3$, we have that $C\left(u_{6} v_{2}\right) \in\left\{i_{3}, i_{4}\right\}$ or there exists a $v \notin\left\{u_{1}, \ldots, u_{6}, v_{1}, v_{2}\right\}$ such that $C\left(u_{6} v\right) \in\left\{i_{3}, i_{4}\right\}$. If $C\left(u_{6} v_{2}\right)=i_{3}$, and so $u_{1} u_{2} u_{3} v_{2} u_{6} u_{5} u_{4}$ is a heterochromatic path of length 6 ; if $C\left(u_{6} v_{2}\right)=i_{4}$, then $u_{4} u_{3} u_{2} u_{1} u_{5} u_{6} v_{2}$ is a heterochromatic path of length 6 . If there is a vertex $v \notin\left\{u_{1}, \ldots, u_{6}, v_{1}, v_{2}\right\}$ such that $C\left(u_{6} v\right) \in\left\{i_{3}, i_{4}\right\}$, then $v_{2} u_{3} u_{2} u_{1} u_{5} u_{6} v$ is a heterochromatic path of length 6 .

(ii.2) $C\left(u_{4} v_{2}\right) \notin\left\{i_{1}, \ldots, i_{6}\right\}$, and let $i_{7}=C\left(u_{4} v_{2}\right)$. Since $P$ is a heterochromatic path of length 5 , we have that $C\left(\left\{u_{1} u_{3}, \ldots, u_{1} u_{6}\right\}\right)-\left\{i_{1}, \ldots, i_{5}, i_{7}\right\} \neq \emptyset$. If $C\left(u_{1} u_{3}\right) \notin$ $\left\{i_{1}, \ldots, i_{5}, i_{7}\right\}$, let $P^{\prime}=u_{2} u_{1} u_{3} u_{4} u_{5} u_{6} v_{1}$; if $C\left(u_{1} u_{4}\right) \notin\left\{i_{1}, \ldots, i_{5}, i_{6}, i_{7}\right\}$, let $P^{\prime}=u_{2} u_{1} u_{4} u_{5}$ $u_{6} v_{1} v_{2}$; if $C\left(u_{1} u_{5}\right) \notin\left\{i_{1}, \ldots, i_{5}, i_{7}\right\}$, let $P^{\prime}=v_{2} u_{4} u_{3} u_{2} u_{1} u_{5} u_{6}$; if $C\left(u_{1} u_{6}\right) \notin\left\{i_{1}, \ldots, i_{5}, i_{7}\right\}$, let $P^{\prime}=v_{2} u_{4} u_{3} u_{2} u_{1} u_{6} u_{5}$. Then, $P^{\prime}$ is a heterochromatic path of length 6 in all these cases. It remains to show that when $C\left(u_{1} u_{4}\right)=i_{6}$, there is a heterochromatic path of length 6. Since $u_{1} u_{2} u_{3} u_{4} v_{2} v_{1}$ is a heterochromatic path of length 5 , we have that $C\left(\left\{u_{1} u_{3}, u_{1} v_{1}, u_{1} v_{2}\right\}\right)-\left\{i_{1}, i_{2}, i_{3}, i_{4}, i_{6}, i_{7}\right\} \neq \emptyset$. If $C\left(u_{1} u_{3}\right) \notin\left\{i_{1}, i_{2}, i_{3}, i_{4}, i_{6}, i_{7}\right\}$, and so $u_{2} u_{1} u_{3} u_{4} v_{2} v_{1} u_{6}$ is a heterochromatic path of length 6 ; if $C\left(u_{1} v_{1}\right) \notin\left\{i_{1}, i_{2}, i_{3}, i_{4}, i_{6}, i_{7}\right\}$, then $v_{2} v_{1} u_{1} u_{2} u_{3} u_{4} u_{5}$ is a heterochromatic path of length 6 ; if $C\left(u_{1} v_{2}\right) \notin\left\{i_{1}, i_{2}, i_{3}, i_{4}, i_{6}\right.$, $\left.i_{7}\right\}$, then $v_{1} v_{2} u_{1} u_{2} u_{3} u_{4} u_{5}$ is a heterochromatic path of length 6 .

(iii) There are vertices $v_{2}, v_{3} \notin\left\{u_{1}, \ldots, u_{6}, v_{1}\right\}$ such that $\left|C\left(\left\{v_{1} v_{2}, v_{2} v_{3}\right\}\right)-C(P)\right|=2$, and $u_{3} u_{4} u_{5} u_{6} v_{1} v_{2} v_{3}$ is a heterochromatic path of length 6 .

(5.3) $k_{0}=3$. So, $\left|C\left(\left\{u_{1} u_{6}, \ldots, u_{4} u_{6}\right\}\right)-\left\{i_{3}, i_{4}, i_{5}\right\}\right|=4$. We have the following three cases:

(i) There is no vertex $v \notin V(P)$ such that $C\left(v_{1} v\right) \notin C(P)$, and so $\mid C\left(\left\{u_{1} v_{1}, \ldots\right.\right.$, $\left.\left.u_{5} v_{1}\right\}\right)-C(P) \mid \geq 2$. Since $\left|C\left(\left\{u_{1} u_{6}, \ldots, u_{4} u_{6}\right\}\right)-\left\{i_{3}, i_{4}, i_{5}\right\}\right|=4$, there is a $u_{7} \notin V(P)$ such that $C\left(u_{6} u_{7}\right)=i_{4}$. If there exists a $u \notin V(P)$ such that $C\left(u u_{1}\right) \notin C(P)$, then $u u_{1} P u_{6}$ is a heterochromatic path of length 6 . If $C\left(u_{3} v_{1}\right) \notin C(P)$, then $u_{1} u_{2} u_{3} v_{1} u_{6} u_{5} u_{4}$ is a heterochromatic path of length 6 . If there exists an $2 \leq i \leq 4$ such that $\mid C\left(\left\{u_{i} v_{1}, u_{i+1} v_{1}\right\}\right)-$ $C(P) \mid=2$, then $u_{1} P u_{i} v_{1} u_{i+1} P u_{6}$ is a heterochromatic path of length 6 . So we shall only show that there is a heterochromatic path of length 6 when $\left|C\left(\left\{u_{1} u_{3}, \ldots, u_{1} u_{6}\right\}\right)-C(P)\right| \geq$ 2 and $C\left(u_{2} v_{1}\right) \notin C(P)$. Let $i_{6}=C\left(u_{2} v_{1}\right)$. Then $C\left(\left\{u_{1} u_{3}, \ldots, u_{1} u_{6}\right\}\right)-\left\{i_{1}, \ldots, i_{5}, i_{6}\right\} \neq$ $\emptyset$. If $C\left(u_{1} u_{3}\right) \notin\left\{i_{1}, \ldots, i_{6}\right\}$, let $P^{\prime}=v_{1} u_{2} u_{1} u_{3} u_{4} u_{5} u_{6}$; if $C\left(u_{1} u_{4}\right) \notin\left\{i_{1}, \ldots, i_{6}\right\}$, let $P^{\prime}=u_{3} u_{2} u_{1} u_{4} u_{5} u_{6} v_{1}$; if $C\left(u_{1} u_{5}\right) \notin\left\{i_{1}, \ldots, i_{6}\right\}$, let $P^{\prime}=u_{4} u_{3} u_{2} u_{1} u_{5} u_{6} u_{7}$; if $C\left(u_{1} u_{6}\right) \notin$ $\left\{i_{1}, \ldots, i_{6}\right\}$, let $P^{\prime}=v_{1} u_{2} u_{1} u_{6} u_{5} u_{4} u_{3}$. Then, $P^{\prime}$ is a heterochromatic path of length 6 in all these cases.

(ii) There is a vertex $v_{2}$ such that $C\left(v_{1} v_{2}\right) \notin C(P)$, and there is no vertex $v \notin$ $\left\{u_{1}, \ldots, u_{6}, v_{1}, v_{2}\right\}$ such that $C\left(v_{1} v_{2}\right) \notin\left\{i_{1}, \ldots, i_{5}\right\} \cup C\left(v_{1} v_{2}\right)$. Let $i_{6}=C\left(v_{1} v_{2}\right)$. Then 
$C\left(\left\{u_{1} v_{2}, \ldots, u_{5} v_{2}\right\}\right)-\left\{i_{1}, \ldots, i_{6}\right\} \neq \emptyset$. If $C\left(u_{1} v_{2}\right) \notin\left\{i_{1}, \ldots, i_{6}\right\}$, then $v_{2} u_{1} P u_{6}$ is a heterochromatic path of length 6 . If $C\left(u_{2} v_{2}\right) \notin\left\{i_{1}, \ldots, i_{6}\right\}$, then $v_{1} v_{2} u_{2} P u_{6}$ is a heterochromatic path of length 6 . If $C\left(u_{3} v_{2}\right) \notin\left\{i_{1}, \ldots, i_{6}\right\}$, then $u_{1} u_{2} u_{3} v_{2} v_{1} u_{6} u_{5}$ is a heterochromatic path of length 6 . If $C\left(u_{5} v_{2}\right) \notin\left\{i_{1}, \ldots, i_{6}\right\}$, then $u_{1} P u_{5} v_{2} v_{1}$ is a heterochromatic path of length 6. Next we shall consider the case when $C\left(u_{4} v_{2}\right) \notin\left\{i_{1}, \ldots, i_{6}\right\}$. Let $i_{7}=C\left(u_{4} v_{2}\right)$. Since $\left|C\left(\left\{u_{1} u_{6}, u_{2} u_{6}, u_{3} u_{6}, u_{4} u_{6}\right\}\right)-\left\{i_{3}, i_{4}, i_{5}\right\}\right|=4$, there is a vertex $u \notin V(P)$ such that $C\left(u_{6} u\right)=i_{4}$. If $u=v_{2}$, i.e., $C\left(u_{6} v_{2}\right)=i_{4}$, then $u_{1} u_{2} u_{3} u_{4} v_{2} u_{6} u_{5}$ is a heterochromatic path of length 6 . It remains to show that there is a heterochromatic path of length 6 if there is a vertex $u \notin\left\{u_{1}, \ldots, u_{6}, v_{1}, v_{2}\right\}$ such that $C\left(u_{6} u\right)=i_{4}$. In this case, since $\left|C\left(\left\{u_{1} u_{3}, \ldots, u_{1} u_{6}\right\}\right)-C(P)\right| \geq 2$, we have that $C\left(\left\{u_{1} u_{4}, u_{1} u_{5}, u_{1} u_{6}\right\}\right)-C(P) \neq \emptyset$. If $C\left(u_{1} u_{4}\right) \notin C(P)$, let $P^{\prime}=u_{3} u_{2} u_{1} u_{4} u_{5} u_{6} v_{1}$; if $C\left(u_{1} u_{5}\right) \notin C(P)$, let $P^{\prime}=u_{4} u_{3} u_{2} u_{1} u_{5} u_{6} u$; if $C\left(u_{1} u_{6}\right) \notin\left\{i_{1}, \ldots, i_{5}, i_{7}\right\}$, let $P^{\prime}=v_{2} u_{4} u_{3} u_{2} u_{1} u_{6} u_{5}$. Then, $P^{\prime}$ is a heterochromatic path of length 6 . Last, we consider the case when $C\left(u_{1} u_{6}\right)=i_{7}$. Since $u_{3} u_{2} u_{1} u_{6} v_{1} v_{2}$ is a heterochromatic path of length 5 , we have $\left|C\left(\left\{u_{1} v_{2}, u_{2} v_{2}, u_{3} v_{2}, u_{6} v_{2}\right\}\right)-\left\{i_{1}, i_{2}, i_{3}, i_{6}, i_{7}\right\}\right| \geq 2$, and so $C\left(\left\{u_{1} v_{2}, u_{3} v_{2}, u_{6} v_{2}\right\}\right)-\left\{i_{1}, i_{2}, i_{3}, i_{6}, i_{7}\right\} \neq \emptyset$. If $C\left(u_{1} v_{2}\right)-\left\{i_{1}, i_{2}, i_{3}, i_{4}, i_{6}, i_{7}\right\} \neq \emptyset$, let $P^{\prime}=v_{1} v_{2} u_{1} P u_{5}$; if $C\left(u_{1} v_{2}\right)=i_{4}$, let $P^{\prime}=u_{3} u_{2} u_{1} v_{2} v_{1} u_{6} u_{5}$; if $C\left(u_{3} v_{2}\right)-\left\{i_{1}, i_{2}, i_{3}, i_{4}, i_{6}, i_{7}\right\}$ $\neq \emptyset$, let $P^{\prime}=u_{1} u_{2} u_{3} v_{2} v_{1} u_{6} u$; if $C\left(u_{3} v_{2}\right)=i_{4}$, let $P^{\prime}=u_{1} u_{2} u_{3} v_{2} v_{1} u_{6} u_{5}$; if $C\left(u_{6} v_{2}\right)-$ $\left\{i_{1}, i_{2}, i_{3}, i_{6}, i_{7}\right\} \neq \emptyset$, let $P^{\prime}=u_{4} u_{3} u_{2} u_{1} u_{6} v_{2} v_{1}$. Then, $P^{\prime}$ is a heterochromatic path of length 6 in all these cases.

(iii) There are vertices $v_{2}, v_{3} \notin\left\{u_{1}, \ldots, u_{6}, v_{1}\right\}$ such that $\left|C\left(\left\{v_{1} v_{2}, v_{2} v_{3}\right\}\right)-C(P)\right|=2$. Let $i_{6}=C\left(v_{1} v_{2}\right)$ and $i_{7}=C\left(v_{2} v_{3}\right)$. If there exists a $v \notin\left\{u_{4}, u_{5}, v_{1}\right\}$ such that $C\left(v_{3} v\right) \notin$ $\left\{i_{3}, \ldots, i_{7}\right\}$, i.e., there exists a $v \notin\left\{u_{4}, u_{5}, u_{6}, v_{1}, v_{2}\right\}$ such that $C\left(v_{3} v\right) \notin\left\{i_{3}, \ldots, i_{7}\right\}$, then $u_{4} u_{5} u_{6} v_{1} v_{2} v_{3} v$ is a heterochromatic path of length 6 . Next we shall only consider the case when $\left|C\left(\left\{u_{4} v_{3}, u_{5} v_{3}, v_{1} v_{3}\right\}\right)-\left\{i_{3}, \ldots, i_{7}\right\}\right| \geq 2$. If $C\left(u_{5} v_{3}\right) \notin\left\{i_{2}, \ldots, i_{7}\right\}$, then $u_{2} u_{3} u_{4} u_{5} v_{3} v_{2} v_{1}$ is a heterochromatic path of length 6 . If $C\left(u_{5} v_{3}\right)=i_{2}$, then $u_{3} u_{4} u_{5} v_{3} v_{2} v_{1}$ is a heterochromatic path of length 5 and $C\left(v_{1} u_{6}\right)=C\left(u_{3} u_{4}\right)$, and so there is a heterochromatic path of length 6 from the cases discussed above. If $C\left(u_{4} v_{3}\right) \notin\left\{i_{1}, \ldots, i_{7}\right\}$, then $v_{1} v_{2} v_{3} u_{4} u_{3} u_{2} u_{1}$ is a heterochromatic path of length 6 . If $C\left(u_{4} v_{3}\right)=i_{1}$, then $u_{2} u_{3} u_{4} v_{3} v_{2} v_{1}$ is a heterochromatic path of length 5 and $C\left(v_{1} u_{6}\right)=C\left(u_{3} u_{4}\right)=i_{3}$, and so there is a heterochromatic path of length 6 because of (5.1) and (5.2). Now it remains to show that there is a heterochromatic path of length 6 when $C\left(u_{4} v_{3}\right)=i_{2}$ and $C\left(v_{1} v_{3}\right) \notin\left\{i_{2}, \ldots, i_{7}\right\}$. Since $\left|C\left(\left\{u_{1} u_{6}, u_{2} u_{6}, u_{3} u_{6}, u_{4} u_{6}\right\}\right)-\left\{i_{3}, \ldots, i_{5}\right\}\right|=4$, there is an $1 \leq x \leq 3$ such that $C\left(u_{x} u_{6}\right) \notin\left\{i_{2}, \ldots, i_{6}\right\}$. If $C\left(u_{x} u_{6}\right) \notin\left\{i_{2}, \ldots, i_{7}\right\}$, then $v_{1} v_{2} v_{3} u_{4} u_{5} u_{6} u_{x}$ is a heterochromatic path of length 6 ; if $C\left(u_{x} u_{6}\right)=i_{7}$, then $v_{2} v_{1} v_{3} u_{4} u_{5} u_{6} u_{x}$ is a heterochromatic path of length 6 . The proof is now complete.

Actually, we can show that for $1 \leq k \leq 5$ any graph $G$ under the color degree condition has a heterochromatic path of length at least $k$, with only one exceptional graph $K_{4}$ for $k=3$, one exceptional graph for $k=4$ and three exceptional graphs for $k=5$, for which $G$ has a heterochromatic path of length at least $k-1$. 


\section{Long heterochromatic paths for $k \geq 8$}

From the above section we know that when $1 \leq k \leq 4$, under the color degree condition $G$ always has a heterochromatic path of length $\left\lceil\frac{3 k}{5}\right\rceil$, and when $5 \leq k \leq 7, G$ always has a heterochromatic path of length $\left\lceil\frac{3 k}{5}\right\rceil+1$. In this section we give our main result and do some preparations for its proof. The detailed proof is left in the next section.

Theorem 3.1 Let $G$ be an edge-colored graph and $k \geq 8$ an integer. Suppose that $d^{c}(v) \geq$ $k$ for every vertex $v$ of $G$. Then $G$ has a heterochromatic path of length at least $\left\lceil\frac{3 k}{5}\right\rceil+1$.

Before proving the result, we will do some preparations, first.

Let $G$ be an edge-colored graph and $k \geq 8$ an integer. Suppose that $d^{c}(v) \geq k$ for every vertex $v$ of $G$. Let $P=u_{1} u_{2} u_{3} \ldots u_{l-1} u_{l} u_{l+1} v_{1} v_{2} \ldots v_{s}$ be a path in $G$ such that

(a) $u_{1} P u_{l+1}$ is a longest heterochromatic path in $G$;

(b) $C\left(u_{l+1} v_{1}\right)=C\left(u_{k_{0}} u_{k_{0}+1}\right)$ and $1 \leq k_{0} \leq l$ is as small as possible, subject to (a);

(c) $v_{1} P v_{s}$ is a heterochromatic path in $G$ with $C\left(u_{1} P u_{l+1}\right) \cap C\left(v_{1} P v_{s}\right)=\varnothing$ and $v_{1} P v_{s}$ is as long as possible, subject to (a) and (b).

Let $i_{j}=C\left(u_{j} u_{j+1}\right)$ for $1 \leq j \leq l$ and $i_{l+j}=C\left(v_{j} v_{j+1}\right)$ for $1 \leq j \leq s-1$, then $C\left(u_{l+1} v_{1}\right)=i_{k_{0}}$. There exist $t_{1} \geq 0$ and $1 \leq x_{1}<x_{2}<\ldots<x_{t_{1}} \leq l$ such that $c\left(\left\{u_{x_{1}} v_{s}, u_{x_{2}} v_{s}, \ldots, u_{x_{t_{1}}} v_{s}\right\}\right)=t_{1}$ and $C\left(\left\{u_{x_{1}} v_{s}, u_{x_{2}} v_{s}, \ldots, u_{x_{t_{1}}} v_{s}\right\}\right)=C\left(\left\{u_{1} v_{s}, \ldots, u_{l} v_{s}\right\}\right)$ $-\left\{i_{1}, \ldots, i_{l+s-1}\right\}$. Let $i_{l+s+j-1}=C\left(u_{x_{j}} v_{s}\right)$ for all $1 \leq j \leq t_{1}$. There also exist $0 \leq$ $t_{2} \leq s-2$ and $1 \leq y_{1}<y_{2}<\ldots<y_{t_{2}} \leq s-2$ such that $C\left(\left\{v_{1} v_{s}, v_{2} v_{s}, \ldots, v_{s-2} v_{s}\right\}\right)-$ $\left\{i_{1}, i_{2}, \ldots, i_{l+s+t_{1}-1}\right\}=C\left(\left\{v_{y_{1}} v_{s}, v_{y_{2}} v_{s}, \ldots, v_{y_{t_{2}} v_{s}}\right\}\right)$ and $c\left(\left\{v_{y_{1}} v_{s}, v_{y_{2}} v_{s}, \ldots, v_{y_{t_{2}} v_{s}}\right\}\right)=t_{2}$. Let $i_{l+s+t_{1}+j-1}=C\left(v_{y_{j}} v_{s}\right)$ for all $1 \leq j \leq t_{2}$.

Then it is easy to get the following Lemmas. In these lemmas we assume that $l=\left\lceil\frac{3 k}{5}\right\rceil$.

Lemma $3.2 s \leq k_{0} \leq 2 l-k$.

Proof. Since $d^{c}\left(u_{l+1}\right) \geq k$ and $u_{1} P u_{l+1}$ is a longest heterochromatic path in $G$, there are at least $k-l$ different edges in $\left\{u_{1} u_{l+1}, u_{2} u_{l+1}, \ldots u_{l-1} u_{l+1}\right\}$ that have different colors which are not in $\left\{i_{1}, i_{2}, \ldots, i_{l}\right\}$. Then we have that $k_{0} \in\{1,2, \ldots,(l-1)-(k-l)+1=2 l-k\}$. On the other hand, if $s>k_{0}$, then $P^{\prime}=u_{k_{0}+1} P v_{k_{0}+1}$ is a heterochromatic path of length $l+1$, a contradiction to the choice of $P$. So, $s \leq k_{0} \leq 2 l-k$.

Lemma 3.3 There are at least $k-l+k_{0}-1$ different colors not in $\left\{i_{k_{0}}, \ldots, i_{l}\right\}$ that belong to $C\left(\left\{u_{1} u_{l+1}, \ldots, u_{l-1} u_{l+1}\right\}\right)$, and $C\left(\left\{u_{1} v_{s}, \ldots, u_{s} v_{s}\right\} \cup\left\{u_{k_{0}-s+1} v_{s}, \ldots, u_{k_{0}} v_{s}\right\} \cup\right.$ $\left.\left\{u_{l-s+2} v_{s}, \ldots, u_{l} v_{s}\right\}\right) \subseteq\left\{i_{1}, \ldots, i_{l+s-1}\right\}$.

Proof. By the choice of $P$, we have $C N\left(u_{l+1}\right)-C\left(\left\{u_{1} u_{l+1}, \ldots, u_{l-1} u_{l+1}\right\}\right) \subseteq\left\{i_{k_{0}}, \ldots, i_{l}\right\}$. Since $d^{c}\left(u_{l+1}\right) \geq k$, there are at least $k-\left(l-k_{0}+1\right)=k-l+k_{0}-1$ different colors not in $\left\{i_{k_{0}}, \ldots, i_{l}\right\}$ that belong to $C\left(\left\{u_{1} u_{l+1}, \ldots, u_{l-1} u_{l+1}\right\}\right)$.

If there exists an $x \in\{1,2, \ldots, s\} \cup\left\{k_{0}-s+1, \ldots, k_{0}\right\} \cup\{l-s+2, l-s+3, \ldots, l\}$ 
such that $u_{x} v_{s}$ has a color not in $\left\{i_{1}, \ldots, i_{l+s-1}\right\}$, then

$$
P^{\prime}= \begin{cases}v_{1} P v_{s} u_{x} P u_{l+x-s+1} & \text { if } x \in\{1,2, \ldots, s\} \\ u_{1} P u_{x} v_{s} P^{-1} u_{x+s} & \text { if } x \in\left\{k_{0}-s+1, \ldots, k_{0}\right\} \\ u_{x-(l-s+1)} P u_{x} v_{s} P^{-1} v_{1} & \text { if } x \in\{l-s+2, l-s+3, \ldots, l\} .\end{cases}
$$

is a heterochromatic path of length $l+1$, a contradiction to the choice of $P$. So, $C\left(\left\{u_{1} v_{s}, \ldots, u_{s} v_{s}, u_{k_{0}-s+1} v_{s}, \ldots, u_{k_{0}} v_{s}, u_{l} v_{s}, \ldots, u_{l-s+2} v_{s}\right\}\right) \subseteq\left\{i_{1}, \ldots, i_{l+s-1}\right\}$.

Lemma $3.4 s<x_{1}<x_{1}+1<x_{2}<x_{2}+1<\ldots<x_{t_{1}} \leq l-s+1, t_{1}+t_{2} \geq k-(l+s-1)$ and $\max \{k-l-2 s+3,0\} \leq t_{1} \leq\left\lceil\frac{l-2 s+1}{2}\right\rceil, 0 \leq t_{2} \leq s-2$.

Proof. It is obvious that $t_{1}+t_{2} \geq k-(l+s-1)$ and $0 \leq t_{2} \leq s-2$, and so $t_{1} \geq$ $k-(l+s-1)-(s-2)=k-l-2 s+3$. From Lemma 3.3, we have that $s<x_{1}<x_{2}<$ $\ldots<x_{t_{1}} \leq l-s+1$. If there exists a $j$ with $1 \leq j \leq t_{1}-1$ such that $u_{x_{j}+1}=u_{x_{j+1}}$, let $P^{\prime}=u_{1} P u_{x_{j}} v_{s} u_{x_{j+1}} P u_{l+1}$, then $P^{\prime}$ a heterochromatic path of length $l+1$, a contradiction to the choice of $P$. So, $s \leq k_{0}<x_{1}<x_{1}+1<x_{2}<x_{2}+1<\ldots<x_{t_{1}} \leq l-s+1$, $t_{1}+t_{2} \geq k-(l+s-1)$ and $\max \{k-l-2 s+3,0\} \leq t_{1} \leq\left\lceil\frac{l-2 s+1}{2}\right\rceil, 0 \leq t_{2} \leq s-2$.

Lemma 3.5 Let $t_{1}=0$. Then $k \equiv 2,4(\bmod 5), k_{0}=s=2 l-k$ and $t_{2}=s-2$ if $k \equiv 4(\bmod 5) ; t_{2} \geq s-3$ if $k \equiv 2(\bmod 5)$. There are exactly $l-1$ different colors not in $\left\{i_{k_{0}}, i_{k_{0}+1}, \ldots, i_{l}\right\}$ that belong to $C\left(u_{1} u_{l+1}, \ldots, u_{l-1} u_{l+1}\right)$, and $C N\left(v_{s}\right)-$ $\left\{u_{s+1} v_{s}, \ldots, u_{l+1} v_{s}, v_{1} v_{s}, \ldots, v_{s-2} v_{s}\right\} \subseteq\left\{i_{k_{0}}, \ldots, i_{l+s-1}\right\}$.

Proof. Since $0=t_{1} \geq k-l-2 s+3$, we have $k-l+3 \leq 2 s \leq 2(2 l-k)=4 l-2 k$. On the other hand, from $(4 l-2 k)-(k-l+3)=5 l-3 k-3$, we have that $5 l-3 k-3<0$ if $k \equiv 0,1,3(\bmod 5), 5 l-3 k-3=0$ if $k \equiv 4(\bmod 5)$ and $5 l-3 k-3=1$ if $k \equiv 2(\bmod 5)$, which implies that $k \equiv 2,4(\bmod 5)$ and $k_{0}=s=2 l-k$. Since $t_{2} \geq k-l-s+1$, we have that $t_{2}=s-2$ if $k \equiv 4(\bmod 5)$ and $t_{2} \geq s-3$ if $k \equiv 2(\bmod 5)$. From Lemma 3.3, there are at least $k-l+k_{0}-1=k-l+s-1=k-l+2 l-k-1=l-1$ different colors not in $\left\{i_{k_{0}}, i_{k_{0}+1}, \ldots, i_{l}\right\}$ that belong to $C\left(u_{1} u_{l+1}, \ldots, u_{l-1} u_{l+1}\right)$. If there exists a $v \notin\left\{u_{s+1}, u_{s+2}, \ldots, u_{l+1}, v_{1}, \ldots, v_{s}\right\}$ such that $v_{s} v$ has a color not in $\left\{i_{k_{0}}, \ldots, i_{l+s-1}\right\}$, then $P^{\prime}=u_{s+1} P v_{s} v$ is a heterochromatic path of length $l+1$, a contradiction to the choice of $P$.

Lemma 3.6 If there exists an $1 \leq x \leq x_{1}-1$ such that $u_{x} u_{l+1}$ has a color in $\left\{i_{l+1}, \ldots\right.$, $\left.i_{l+s-\left\lceil\frac{x_{1}-1}{2}\right\rceil}\right\} \cup\left\{i_{l+\left\lceil\frac{s+x_{1}-t_{2}}{2}\right\rceil-2}, i_{l+\left\lceil\frac{s+x_{1}-t_{2}}{2}\right\rceil-1}, \ldots, i_{l+s-1}\right\}$, then there is a heterochromatic path $P^{\prime}$ of length $l+1$ in $G$.

Proof. First, note that $\left(l+\left\lceil\frac{s+x_{1}-t_{2}}{2}\right\rceil-2\right)-\left(l+s-\left\lceil\frac{x_{1}-1}{2}\right\rceil\right)=\left\lceil\frac{s+x_{1}-t_{2}}{2}\right\rceil-2-s+\left\lceil\frac{x_{1}-1}{2}\right\rceil \geq$ $\left\lceil\frac{x_{1}+2}{2}\right\rceil+\left\lceil\frac{x_{1}-1}{2}\right\rceil-s-2=x_{1}+1-s-2 \geq 0$.

If there exists an $1 \leq x \leq x_{1}-1$ such that $u_{x} u_{l+1}$ has a color in $\left\{i_{l+1}, \ldots, i_{l+s-\left\lceil\frac{x_{1}-1}{2}\right\rceil}\right\}$, then let

$$
P^{\prime}= \begin{cases}v_{s-\left\lceil\frac{x_{1}-1}{2}\right\rceil+1} P v_{s} u_{x_{1}} P u_{l+1} u_{x} P^{-1} u_{x-\left(\left\lfloor\frac{x_{1}-1}{2}\right\rfloor+1\right)+1} & \text { if }\left\lfloor\frac{x_{1}-1}{2}\right\rfloor+1 \leq x \leq x_{1}-1 ; \\ v_{s-\left\lceil\frac{x_{1}-1}{2}\right\rceil+1} P v_{s} u_{x_{1}} P u_{l+1} u_{x} P u_{x+\left(\left\lfloor\frac{x_{1}-1}{2}\right\rfloor+1\right)-1} & \text { if } 1 \leq x \leq\left\lfloor\frac{x_{1}-1}{2}\right\rfloor .\end{cases}
$$


Since $\left\lfloor\frac{x_{1}-1}{2}\right\rfloor+\left(\left\lfloor\frac{x_{1}-1}{2}\right\rfloor+1\right)-1=2\left\lfloor\frac{x_{1}-1}{2}\right\rfloor \leq x_{1}-1, P^{\prime}$ is a heterochromatic path of length $l+1$.

If there exists an $1 \leq x \leq x_{1}-1$ such that $u_{x} u_{l+1}$ has a color $i_{l+y} \in\left\{i_{l+\left\lceil\frac{s+x_{1}-t_{2}}{2}\right\rceil-2}\right.$, $\left.i_{l+\left\lceil\frac{s+x_{1}-t_{2}}{2}\right\rceil-1}, \ldots, i_{l+s-1}\right\}$, then since

$$
\begin{aligned}
t_{2}-\left[(s-2)-\left(\left\lceil\frac{s+x_{1}-t_{2}}{2}\right\rceil-2\right)\right] & =t_{2}-s+\left\lceil\frac{s+x_{1}-t_{2}}{2}\right\rceil \geq t_{2}-s+\frac{s+x_{1}-t_{2}}{2} \\
& =\frac{t_{2}-s+x_{1}}{2-l-s+1-t_{1}-s+s+1} 2 \frac{k-l}{2} \\
& \geq \frac{k-l-s+2}{2}-\frac{1}{2}\left\lceil\frac{l-2 s+1}{2}\right\rceil \\
& \geq \frac{k-l-s+2}{2}-\frac{l-2 s+2}{4} \\
& =\frac{2 k-3 l+2}{4}>0
\end{aligned}
$$

and $y_{t_{2}-\left[(s-2)-\left(\left\lceil\frac{s+x_{1}-t_{2}}{2}\right\rceil-2\right)\right]} \leq\left\lceil\frac{s+x_{1}-t_{2}}{2}\right\rceil-2$, there are some $y^{\prime} \in\left\{1,2, \ldots,\left\lceil\frac{s-t_{2}-1}{2}\right\rceil\right\} \cup$ $\left\{\left\lceil\frac{s+x_{1}-t_{2}}{2}\right\rceil-2,\left\lceil\frac{s+x_{1}-t_{2}}{2}\right\rceil-3, \ldots,\left\lceil\frac{s+x_{1}-t_{2}}{2}\right\rceil-\left\lfloor\frac{s-t_{2}-1}{2}\right\rfloor-1\right\}$ such that $y^{\prime} \in\left\{y_{1}, y_{2}, \ldots, y_{t_{2}}\right\}$. Let

$$
P_{1}= \begin{cases}v_{y^{\prime}+\left\lceil\frac{s+x_{1}-t_{2}}{2}\right\rceil-\left\lceil\frac{s-t_{2}-1}{2}\right\rceil-2} P^{-1} v_{y^{\prime}} v_{s} & \text { if } y^{\prime} \in\left\{1,2, \ldots,\left\lceil\frac{s-t_{2}-1}{2}\right\rceil\right\} \\ v_{y^{\prime}-\left\lceil\frac{s+x_{1}-t_{2}}{2}\right\rceil+\left\lceil\frac{s-t_{2}-1}{2}\right\rceil+2} P v_{y^{\prime}} v_{s} & \text { if } y^{\prime} \in\left\{\left\lceil\frac{s+x_{1}-t_{2}}{2}\right\rceil-2, \ldots,\right. \\ & \left.\left\lceil\frac{s+x_{1}-t_{2}}{2}\right\rceil-\left\lfloor\frac{s-t_{2}-1}{2}\right\rfloor-1\right\} .\end{cases}
$$

Note that if $y^{\prime} \in\left\{1,2, \ldots,\left\lceil\frac{s-t_{2}-1}{2}\right\rceil\right\}$, we have that $y^{\prime}+\left\lceil\frac{s+x_{1}-t_{2}}{2}\right\rceil-\left\lceil\frac{s-t_{2}-1}{2}\right\rceil-2 \leq\left\lceil\frac{s-t_{2}-1}{2}\right\rceil+$ $\left\lceil\frac{s+x_{1}-t_{2}}{2}\right\rceil-\left\lceil\frac{s-t_{2}-1}{2}\right\rceil-2=\left\lceil\frac{s+x_{1}-t_{2}}{2}\right\rceil-2$, and if $y^{\prime} \in\left\{\left\lceil\frac{s+x_{1}-t_{2}}{2}\right\rceil-2, \ldots,\left\lceil\frac{s+i_{1}-t_{2}}{2}\right\rceil-\left\lfloor\frac{s-t_{2}-1}{2}\right\rfloor-\right.$ $1\}$, we have that $y^{\prime}-\left\lceil\frac{s+x_{1}-t_{2}}{2}\right\rceil+\left\lceil\frac{s-t_{2}-1}{2}\right\rceil+2 \geq\left\lceil\frac{s+x_{1}-t_{2}}{2}\right\rceil-\left\lfloor\frac{s-t_{2}-1}{2}\right\rfloor-1-\left\lceil\frac{s+x_{1}-t_{2}}{2}\right\rceil+$ $\left\lceil\frac{s-t_{2}-1}{2}\right\rceil+2 \geq 1$. Then, $P_{1}$ is a heterochromatic path of length $\left\lceil\frac{s+x_{1}-t_{2}}{2}\right\rceil-\left\lceil\frac{s-t_{2}-1}{2}\right\rceil-1$ with colors not in $\left\{i_{1}, i_{2}, \ldots, i_{l}, i_{l+y}, i_{l+s}\right\}$. Let

$$
P^{\prime}= \begin{cases}P_{1} u_{x_{1}} P u_{l+1} u_{x} P^{-1} u_{x-\left(x_{1}-\left\lceil\frac{s+x_{1}-t_{2}}{2}\right\rceil+\left\lceil\frac{s-t_{2}-1}{2}\right\rceil\right)+1} & \text { if } x_{1}-\left\lceil\frac{s+x_{1}-t_{2}}{2}\right\rceil+ \\ & \left\lceil\frac{s-t_{2}-1}{2}\right\rceil \leq x \leq x_{1}-1 \\ P_{1} u_{x_{1}} P u_{l+1} u_{x} P u_{x+\left(x_{1}-\left\lceil\frac{s+x_{1}-t_{2}}{2}\right\rceil+\left\lceil\frac{s-t_{2}-1}{2}\right\rceil\right)-1} & \text { if } 1 \leq x \leq x_{1}- \\ & \left\lceil\frac{s+x_{1}-t_{2}}{2}\right\rceil+\left\lceil\frac{s-t_{2}-1}{2}\right\rceil-1\end{cases}
$$

Since $2\left(x_{1}-\left\lceil\frac{s+x_{1}-t_{2}}{2}\right\rceil+\left\lceil\frac{s-t_{2}-1}{2}\right\rceil-1\right)=2 x_{1}-2\left\lceil\frac{s+x_{1}-t_{2}}{2}\right\rceil+2\left\lceil\frac{s-t_{2}-1}{2}\right\rceil-2 \leq 2 x_{1}-(s+$ $\left.x_{1}-t_{2}\right)+\left(s-t_{2}\right)-2=x_{1}-2, P^{\prime}$ is a heterochromatic path of length $l+1$.

Lemma 3.7 Let $t_{1}=0, k \geq 8, s+1 \leq x \leq l-s+2$ and $C\left(u_{x} v_{s}\right)=i_{1}$. If there exists an $2 \leq x^{\prime} \leq x-1$ such that $u_{x^{\prime}} u_{l+1}$ has a color in $\left\{i_{l+1}, \ldots, i_{l+s-\left\lceil\frac{x}{2}\right\rceil}, i_{l+\left\lceil\frac{x}{2}\right\rceil-1}, \ldots, i_{l+s-1}\right\}$ then there is a heterochromatic path $P^{\prime}$ of length $l+1$ in $G$.

Proof. Since $t_{1}=0$, we have that $k \equiv 2,4(\bmod 5)$ and $s=2 l-k$, and that $t_{2}=s-2$ if $k \equiv 4(\bmod 5) ; t_{2} \geq s-3$ if $k \equiv 2(\bmod 5)$ from Lemma 3.5 .

If there exists an $2 \leq x^{\prime} \leq x-1$ such that $u_{x^{\prime}} u_{l+1}$ has a color in $\left\{i_{l+1}, \ldots, i_{l+s-\left\lceil\frac{x}{2}\right\rceil}\right\}$, then let

$$
P^{\prime}= \begin{cases}v_{s-\left\lceil\frac{x}{2}\right\rceil+1} P v_{s} u_{x} P u_{l+1} u_{x}^{\prime} P^{-1} u_{x^{\prime}-\left\lfloor\frac{x}{2}\right\rfloor+1} & \text { if }\left\lfloor\frac{x}{2}\right\rfloor+1 \leq x^{\prime} \leq x-1 \\ v_{s-\left\lceil\frac{x}{2}\right\rceil+1} P v_{s} u_{x} P u_{l+1} u_{x}^{\prime} P u_{x^{\prime}+\left\lfloor\frac{x}{2}\right\rfloor-1} & \text { if } 2 \leq x^{\prime} \leq\left\lfloor\frac{x}{2}\right\rfloor\end{cases}
$$


Note that if $2 \leq x^{\prime} \leq\left\lfloor\frac{x}{2}\right\rfloor$, then $x^{\prime}+\left\lfloor\frac{x}{2}\right\rfloor-1 \leq 2\left\lfloor\frac{x}{2}\right\rfloor-1 \leq x-1$, and so $P^{\prime}$ is a heterochromatic path of length $l+1$.

If there exists an $2 \leq x^{\prime} \leq x-1$ such that $u_{x^{\prime}} u_{l+1}$ has a color in $\left\{i_{l+\left\lceil\frac{x}{2}\right\rceil-1}, \ldots, i_{l+s-1}\right\}$, since $t_{2}=s-2$ if $k \equiv 4(\bmod 5)$ and $t_{2} \geq s-3$ if $k \equiv 2(\bmod 5)$, and $k \geq 8$, we have $t_{2} \geq 1$. On the other hand, if $k \equiv 2(\bmod 5)$, then $2 \leq\left\lceil\frac{s+1}{2}\right\rceil-1 \leq\left\lceil\frac{x}{2}\right\rceil-1 \leq$ $\left\lceil\frac{l-s+2}{2}\right\rceil-1=\left\lceil\frac{k-l+2}{2}\right\rceil-1=\left\lceil\frac{2 s+(k-l-2(2 l-k)+2)}{2}\right\rceil-1=s-2$, and so $C\left(\left\{v_{1} v_{s}, v_{\left\lceil\frac{x}{2}\right\rceil-1} v_{s}\right\}\right) \nsubseteq$ $\left\{i_{1}, i_{2}, \ldots, i_{l+s-1}\right\}$. Let

$$
\begin{aligned}
& P_{1}= \begin{cases}v_{1} P v_{\left\lceil\frac{x}{2}\right\rceil-1} v_{s} & \text { if } v_{\left\lceil\frac{x}{2}\right\rceil-1} v_{s} \text { has a color not in }\left\{i_{1}, i_{2}, \ldots, i_{l+s-1}\right\} ; \\
v_{\left\lceil\frac{x}{2}\right\rceil-1} P^{-1} v_{1} v_{s} & \text { if } v_{1} v_{s} \text { has a color not in }\left\{i_{1}, i_{2}, \ldots, i_{l+s-1}\right\} .\end{cases} \\
& P^{\prime}= \begin{cases}P_{1} u_{x} P u_{l+1} u_{x^{\prime}} P^{-1} u_{x^{\prime}-\left\lfloor\frac{x}{2}\right\rfloor+1} & \text { if }\left\lfloor\frac{x}{2}\right\rfloor+1 \leq x^{\prime} \leq x-1 ; \\
P_{1} u_{x} P u_{l+1} u_{x^{\prime}} P u_{x^{\prime}+\left\lfloor\frac{x}{2}\right\rfloor-1} & \text { if } 2 \leq x^{\prime} \leq\left\lfloor\frac{x}{2}\right\rfloor .\end{cases}
\end{aligned}
$$

Note that if $2 \leq x^{\prime} \leq\left\lfloor\frac{x}{2}\right\rfloor$, then $x^{\prime}+\left\lfloor\frac{x}{2}\right\rfloor-1 \leq 2\left\lfloor\frac{x}{2}\right\rfloor-1 \leq x-1$, and so $P^{\prime}$ is a heterochromatic path of length $l+1$.

Lemma 3.8 Let $t_{1}=0,\left|C\left(v_{1} v_{s}, \ldots, v_{s-2} v_{s}\right)-\left\{i_{2}, \ldots, i_{l+s-1}\right\}\right|=s-2, k \geq 8$ and $C\left(u_{x} v_{s}\right)=i_{2}$. If there exists an $3 \leq x^{\prime} \leq x-1$ such that $u_{x^{\prime}} u_{l+1}$ has a color in $\left\{i_{l+1}, \ldots, i_{l+s-\left\lceil\frac{x+1}{2}\right\rceil}, i_{l+\left\lceil\frac{x+1}{2}\right\rceil-1}, \ldots, i_{l+s-1}\right\}$, then there is a heterochromatic path of length $l+1$.

Proof. If there exists an $3 \leq x^{\prime} \leq x-1$ such that $u_{x^{\prime}} u_{l+1}$ has a color in $\left\{i_{l+1}, \ldots\right.$, $\left.i_{l+s-\left\lceil\frac{x+1}{2}\right\rceil}\right\}$, then let

$$
P^{\prime}= \begin{cases}v_{s-\left\lceil\frac{x+1}{2}\right\rceil+1} P v_{s} u_{x} P u_{l+1} u_{x^{\prime}} P^{-1} u_{x^{\prime}-\left\lfloor\frac{x+1}{2}\right\rfloor+2} & \text { if }\left\lfloor\frac{x+1}{2}\right\rfloor+1 \leq x^{\prime} \leq x-1 \\ v_{s-\left\lceil\frac{x+1}{2}\right\rceil+1} P v_{s} u_{x} P u_{l+1} u_{x^{\prime}} P u_{x^{\prime}+\left\lfloor\frac{x+1}{2}\right\rfloor-2} & \text { if } 3 \leq x^{\prime} \leq\left\lfloor\frac{x+1}{2}\right\rfloor\end{cases}
$$

Note that if $3 \leq x^{\prime} \leq\left\lfloor\frac{x+1}{2}\right\rfloor$, then $x^{\prime}+\left\lfloor\frac{x+1}{2}\right\rfloor-2 \leq 2\left\lfloor\frac{x+1}{2}\right\rfloor-2 \leq x-1$, and so $P^{\prime}$ is a heterochromatic path of length $l+1$.

If there exists an $x^{\prime}$ with $3 \leq x^{\prime} \leq x-1$ such that $u_{x} u_{l+1}$ has a color in $\left\{i_{l+\left\lceil\frac{x+1}{2}\right\rceil-1}, \ldots\right.$, $\left.i_{l+s-1}\right\}$, then let

$$
P^{\prime}= \begin{cases}v_{\left\lceil\frac{x+1}{2}\right\rceil-1} P^{-1} v_{1} v_{s} u_{x} P u_{l+1} u_{x^{\prime}} P^{-1} u_{x^{\prime}-\left\lfloor\frac{x+1}{2}\right\rfloor+2} & \text { if }\left\lfloor\frac{x+1}{2}\right\rfloor+1 \leq x^{\prime} \leq x-1 \\ v_{\left\lceil\frac{x+1}{2}\right\rceil-1} P^{-1} v_{1} v_{s} u_{x} P u_{l+1} u_{x^{\prime}} P u_{x^{\prime}+\left\lfloor\frac{x+1}{2}\right\rfloor-2} & \text { if } 3 \leq x^{\prime} \leq\left\lfloor\frac{x+1}{2}\right\rfloor\end{cases}
$$

and so $P^{\prime}$ is a heterochromatic path of length $l+1$.

Lemma 3.9 Let $t_{1} \geq 1, k \geq 8$ and $k \equiv 1,2,4(\bmod 5)$. Then there is no $x_{j}\left(1 \leq j \leq t_{1}\right)$ such that $C\left(\left\{u_{1} u_{l+1}, u_{2} u_{l+1}, \ldots, u_{x_{j}-1} u_{l+1}\right\}\right)-\left\{i_{1}, i_{2}, \ldots, i_{l}, i_{l+1}, \ldots, i_{l+s-1}, i_{l+s+j-1}\right\} \neq \emptyset$.

Proof. Suppose there is some $x_{j}\left(1 \leq j \leq t_{1}\right)$ such that $C\left(\left\{u_{1} u_{l+1}, u_{2} u_{l+1}, \ldots, u_{x_{j}-1} u_{l+1}\right\}\right)$ $-\left\{i_{1}, i_{2}, \ldots, i_{l}, i_{l+1}, \ldots, i_{l+s-1}, i_{l+s+j-1}\right\} \neq \emptyset$. Let $x_{j_{0}}$ be the one of such $x_{j}$ with the smallest subscript, and $u_{x^{\prime}} u_{l+1}\left(1 \leq x^{\prime} \leq x_{j_{0}}-1\right)$ has a color not in $\left\{i_{1}, i_{2}, \ldots, i_{l}, i_{l+1}, \ldots\right.$, 
$\left.i_{l+s-1}, i_{l+s+j_{0}-1}\right\}$. We distinguish the following three cases.

Case $1 j_{0}=1$. Let

$$
P^{\prime}= \begin{cases}v_{1} P v_{s} u_{x_{1}} P u_{l+1} u_{x^{\prime}} P^{-1} u_{x^{\prime}-\left(x_{1}-s\right)+1} & \text { if } x_{1}-s \leq x^{\prime} \leq x_{1}-1 \\ v_{1} P v_{s} u_{x_{1}} P u_{l+1} u_{x^{\prime}} P u_{x^{\prime}+\left(x_{1}-s\right)-1} & \text { if } 1 \leq x^{\prime} \leq x_{1}-s-1 .\end{cases}
$$

Since $t_{1} \geq k-l-2 s+3$ by Lemma 3.4, we have that $\left(x_{1}-s-1\right)+\left(x_{1}-s-1\right)=$ $x_{1}+\left(x_{1}-2 s-2\right) \leq x_{1}+\left(l-s+1-2\left(t_{1}-1\right)-2 s-2\right)=x_{1}+\left(l-2 t_{1}-3 s+1\right) \leq$ $x_{1}+(l-2 k+2 l+4 s-6-3 s+1)=x_{1}+(3 l-2 k+s-5) \leq x_{1}+(3 l-2 k+2 l-k-5)=$ $x_{1}+(5 l-3 k-5)=x_{1}+\left(5\left\lceil\frac{3 k}{5}\right\rceil-3 k-5\right) \leq x_{1}-1$. So, $P^{\prime}$ is a heterochromatic path of length $l+1$, a contradiction to the choice of $P$.

Case $2 \quad j_{0}=2$. So $t_{1} \geq 2$. Since $t_{1} \geq k-l-2 s+3 \geq k-l-4 l+2 k+$ $3=3 k-5 l+3=3 k-5\left\lceil\frac{3 k}{5}\right\rceil+3$, we have that $k-l-2 s+3<2$ if and only if $s=2 l-k$ when $k \equiv 1,2,4(\bmod 5)$, or $s=2 l-k-1$ when $k \equiv 2(\bmod 5)$. Then $t_{1} \geq \max \{k-l-2 s+3,2\}=2$ if $s=2 l-k$ when $k \equiv 1,2,4(\bmod 5)$, or $s=2 l-k-1$ when $k \equiv 2(\bmod 5)$. Otherwise, $t_{1} \geq k-l-2 s+3$.

We first consider the case when $u_{x^{\prime}} u_{l+1}$ has a color $l+s$. Then $1 \leq x^{\prime} \leq x_{2}-1$. Let

$$
P^{\prime}= \begin{cases}v_{1} P v_{s} u_{x_{2}} P u_{l+1} u_{x^{\prime}} P^{-1} u_{x^{\prime}-\left(x_{2}-s\right)+1} & \text { if } x_{2}-s \leq x^{\prime} \leq x_{2}-1 ; \\ v_{1} P v_{s} u_{x_{2}} P u_{l+1} u_{x^{\prime}} P u_{x^{\prime}+\left(x_{2}-s\right)-1} & \text { if } 1 \leq x^{\prime} \leq x_{2}-s-1 .\end{cases}
$$

Note that $\left(x_{2}-s-1\right)+x_{2}-s-1=x_{2}+\left(x_{2}-2 s-2\right) \leq x_{2}+\left(l-s+1-2\left(t_{1}-2\right)-2 s-2\right)=$ $x_{2}+\left(l-3 s-2 t_{1}+3\right) \leq x_{2}+l-3 s-2 \max \{k-l-2 s+3,2\}+3$.

If $k \equiv 1,4(\bmod 5)$, then

$$
\begin{aligned}
& x_{2}+l-3 s-2 \max \{k-l-2 s+3,2\}+3 \\
= & \begin{cases}x_{2}+l-3 s-2 k+2 l+4 s-3 & \text { if } s \leq 2 l-k-1 ; \\
x_{2}+l-3 s-1 & \text { if } s=2 l-k . \\
x_{2}+3 l-2 k+2 l-k-1-3 & \text { if } s \leq 2 l-k-1 ; \\
x_{2}+l-6 l+3 k-1 & \text { if } s=2 l-k .\end{cases} \\
= & \left\{\begin{array}{l}
x_{2}+5\left\lceil\frac{3 k}{5}\right\rceil-3 k-4 \\
x_{2}+3 k-5\left\lceil\frac{3 k}{5}\right\rceil-1
\end{array} \text { if } s=2 l-k-1 ;\right. \\
\leq & x_{2}-1 .
\end{aligned}
$$

If $k \equiv 2(\bmod 5)$, then

$$
\begin{aligned}
& x_{2}+l-3 s-2 \max \{k-l-2 s+3,2\}+3 \\
& = \begin{cases}x_{2}+l-3 s-2 k+2 l+4 s-3 & \text { if } s \leq 2 l-k-2 ; \\
x_{2}+l-3 s-1 & \text { if } s=2 l-k, 2 l-k-1 .\end{cases} \\
& \leq \begin{cases}x_{2}+3 l-2 k+2 l-k-2-3 & \text { if } s \leq 2 l-k-2 ; \\
x_{2}+l-6 l+3 k+3-1 & \text { if } s=2 l-k, 2 l-k-1 .\end{cases} \\
& =\left\{\begin{array}{l}
x_{2}+5\left\lceil\frac{3 k}{5}\right\rceil-3 k-5 \text { if } s \leq 2 l-k-2 ; \\
x_{2}+3 k-5\left\lceil\frac{3 k}{5}\right\rceil+2 \text { if } s=2 l-k, 2 l-k-1 .
\end{array}\right. \\
& \leq x_{2}-1 \text {. }
\end{aligned}
$$

So, $P^{\prime}$ is a heterochromatic path of length $l+1$, a contradiction to the choice of $P$.

Next we consider the case when $u_{x^{\prime}} u_{l+1}$ has a color not in $\left\{i_{1}, i_{2}, \ldots, i_{l}, i_{l+1}, \ldots\right.$, 
$\left.i_{l+s-1}, i_{l+s}, i_{l+s+1}\right\}$. Then $x_{1} \leq x^{\prime} \leq x_{2}-1$. Let $P^{\prime}=v_{1} P v_{s} u_{x_{2}} P u_{l+1} u_{x^{\prime}} P^{-1} u_{x^{\prime}-\left(x_{2}-s\right)+1}$. Note that $x^{\prime}-\left(x_{2}-s\right)+1 \geq x_{1}-x_{2}+s+1 \geq s+1-\left(l-s+1-2\left(t_{1}-2\right)\right)+s+1$ $=3 s+2 t_{1}-l-3 \geq 3 s+2 \max \{k-l-2 s+3,2\}-l-3$.

If $k \equiv 1,4(\bmod 5)$, then

$$
\begin{aligned}
& 3 s+2 \max \{k-l-2 s+3,2\}-l-3 \\
= & \begin{cases}3 s+2 k-2 l-4 s+6-l-3 & \text { if } s \leq 2 l-k-1 ; \\
3 s+4-l-3 & \text { if } s=2 l-k . \\
2 k-3 l-2 l+k+1+3 & \text { if } s \leq 2 l-k-1 \\
6 l-3 k-l+1 & \text { if } s=2 l-k .\end{cases} \\
= & \begin{cases}3 k-5\left\lceil\frac{3 k}{5}\right\rceil+4 & \text { if } s \leq 2 l-k-1 \\
5\left\lceil\frac{3 k}{5}\right\rceil-3 k+1 & \text { if } s=2 l-k .\end{cases} \\
\geq & 1 .
\end{aligned}
$$

If $k \equiv 2(\bmod 5)$, then

$$
\begin{aligned}
& 3 s+2 \max \{k-l-2 s+3,2\}-l-3 \\
& = \begin{cases}3 s+2 k-2 l-4 s+6-l-3 & \text { if } s \leq 2 l-k-2 ; \\
3 s+4-l-3 & \text { if } s=2 l-k, 2 l-k-1 .\end{cases} \\
& \geq \begin{cases}2 k-3 l-2 l+k+2+3 & \text { if } s \leq 2 l-k-2 \\
6 l-3 k-3-l+1 & \text { if } s=2 l-k, 2 l-k-1 .\end{cases} \\
& = \begin{cases}3 k-5\left\lceil\frac{3 k}{5}\right\rceil+5 & \text { if } s \leq 2 l-k-2 \\
5\left\lceil\frac{3 k}{5}\right\rceil-3 k-2 & \text { if } s=2 l-k, 2 l-k-1 .\end{cases} \\
& \geq 1 \text {. }
\end{aligned}
$$

So, $P^{\prime}$ is a heterochromatic path of length $l+1$, a contradiction to the choice of $P$.

Case $33 \leq j_{0} \leq t_{1}$. So $t_{1} \geq 3$. Since $t_{1} \geq k-l-2 s+3 \geq 3 k-5\left\lceil\frac{3 k}{5}\right\rceil+3$, we have that $k-l-2 s+3<3$ if and only if $s=2 l-k$ when $k \equiv 1,2,4(\bmod 5)$, or $s=2 l-k-1$ when $k \equiv 2,4(\bmod 5)$. Then $t_{1} \geq \max \{k-l-2 s+3,3\}=3$ if $s=2 l-k$ when $k \equiv 1,2,4(\bmod 5)$ or $s=2 l-k-1$ when $k \equiv 2,4(\bmod 5)$; otherwise, $t_{1} \geq k-l-2 s+3$.

Case 3.1 $C\left(u_{x^{\prime}} u_{l+1}\right)=i_{l+s+j_{0}-2}$. Then $x_{j_{0}-2} \leq x^{\prime} \leq x_{j_{0}}-1$. Note that $x_{j_{0}}-x_{j_{0}-2} \leq$ $\left(l-s+1-2\left(t_{1}-j_{0}\right)\right)-\left(x_{1}+2\left(j_{0}-3\right)\right)=l-s-x_{1}-2 t_{1}+7 \leq l-s-(s+1)-2 \max \{k-$ $l-2 s+3,3\}+7=l-2 s-2 \max \{k-l-2 s+3,3\}+6$.

If $k \equiv 1(\bmod 5)$, then

$$
\begin{aligned}
& x_{j_{0}}-x_{j_{0}-2} \\
\leq & l-2 s-2 \max \{k-l-2 s+3,3\}+6 \\
= & \begin{cases}l-2 s-2 k+2 l+4 s-6+6 & \text { if } s \leq 2 l-k-1 ; \\
l-2 s-6+6 & \text { if } s=2 l-k .\end{cases} \\
\leq & \begin{cases}s+(3 l-2 k+2 l-k-1) & \text { if } s \leq 2 l-k-1 \\
s+(l-6 l+3 k) & \text { if } s=2 l-k . \\
s+\left(5\left\lceil\frac{3 k}{5}\right\rceil-3 k-1\right) & \text { if } s \leq 2 l-k-1 \\
s+\left(3 k-5\left\lceil\frac{3 k}{5}\right\rceil\right) & \text { if } s=2 l-k . \\
s+1 & \text { if } s \leq 2 l-k-1 ; \\
s-2 & \text { if } s=2 l-k .\end{cases}
\end{aligned}
$$


If $k \equiv 2,4(\bmod 5)$, then

$$
\begin{aligned}
& x_{j_{0}}-x_{j_{0}-2} \\
\leq & l-2 s-2 \max \{k-l-2 s+3,3\}+6 \\
= & \left\{\begin{array}{lr}
l-2 s-2 k+2 l+4 s-6+6 & \text { if } s \leq 2 l-k-2 ; \\
l-2 s-6+6 & \text { if } s=2 l-k, 2 l-k-1 . \\
s+(3 l-2 k+2 l-k-2) & \text { if } s \leq 2 l-k-2 ; \\
s+(l-6 l+3 k+3) & \text { if } s=2 l-k, 2 l-k-1 . \\
s+\left(5\left\lceil\frac{3 k}{5}\right\rceil-3 k-2\right) & \text { if } s \leq 2 l-k-2 ; \\
s+\left(3 k-5\left\lceil\frac{3 k}{5}\right\rceil+3\right) & \text { if } s=2 l-k, 2 l-k-1 .
\end{array}\right. \\
\leq & \begin{cases}s+2 \text { if } s \leq 2 l-k-2 \text { and } k \equiv 2(\bmod 5) ; \\
s+1 \quad \text { if } s \leq 2 l-k-2 \text { and } k \equiv 4(\bmod 5) ; \\
s \quad \text { if } s=2 l-k, 2 l-k-1 .\end{cases}
\end{aligned}
$$

If $x_{j_{0}}-x^{\prime} \leq s$, let $P^{\prime}=v_{1} P v_{s} u_{x_{0}} P u_{l+1} u_{x^{\prime}} P^{-1} u_{x^{\prime}-\left(x_{j_{0}}-s\right)+1}$, then $P^{\prime}$ is a heterochromatic path of length $l+1$, a contradiction to the choice of $P$. So, $x_{j_{0}}-x_{j_{0}-2} \geq x_{j_{0}}-x^{\prime} \geq s+1$. Then we have the following cases:

Case 3.1.1 $k \equiv 1(\bmod 5)$. Then $x_{j_{0}-2}=s+1+2\left(j_{0}-3\right)=s+2 j_{0}-5$, $x_{j_{0}}=l-s+1-2\left(t_{1}-j_{0}\right)=l-s-2 t_{1}+2 j_{0}+1, x^{\prime}=x_{j_{0}-2}=x_{j_{0}}-s-1, s=2 l-k-1$ and $t_{1}=k-l-2 s+3=k-l-4 l+2 k+2+3=3 k-5 l+5=3 k-5\left\lceil\frac{3 k}{5}\right\rceil+5=3$. So, $t_{2} \geq k-(l+s-1)-t_{1}=s+(k-l-2 s+1)-3=s-2$, and then $t_{2}=s-2$. Hence, $j_{0}=t_{1}=3, x_{1}=s+1, x_{3}=l-s+1$, and $4 \leq x_{3}-x_{1}=l-2 s=l-4 l+2 k+2=$ $2 k-3 l+2=s+(2 k-3 l-2 l+k+3)=s+(3 k-5 l+3)=s+1$ and so $s \geq 3$. On the other hand, we have that $s \leq k_{0} \leq 2 l-k=s+1=x_{1}$ which implies that $k_{0}-s+1 \leq 2<s$. Then, $k_{0}<x=s+1$ from Lemma 3.3, and we get that $k_{0}=s$.

If there exists an $x$ with $1 \leq x \leq s$ such that $u_{x} u_{l+1}$ has a color not in $\left\{i_{k_{0}}, i_{k_{0}+1}, \ldots\right.$, $\left.i_{l}, i_{l+1}, \ldots, i_{l+s-1}, i_{l+s}\right\}$, let $P^{\prime}=v_{1} P v_{s} u_{s+1} P u_{l+1} u_{x}$, then $P^{\prime}$ is a heterochromatic path of length $l+1$, a contradiction to the choice of $P$.

Otherwise, there are at least $s-\left((l-1)-\left(k-l+k_{0}-1\right)\right)=s-2 l+k+k_{0}=s-s-1+s=$ $s-1$ colors in $\left\{i_{l+1}, i_{l+2}, \ldots, i_{l+s-1}\right\}$ that belong to $C\left(\left\{u_{1} u_{l+1}, u_{2} u_{l+1}, \ldots, u_{s} u_{l+1}\right\}\right)$. So, there is an $x$ with $1 \leq x \leq s$ such that $u_{x} u_{l+1}$ has a color $i_{l+s-1}$. Since $s \geq 3$ and $t_{2}=s-2 \geq 1$, we have that $v_{1} v_{s} \in E(G)$ and $v_{1} v_{s}$ has color $i_{l+s+3}$. So, $P^{\prime}=$ $v_{s-1} P^{-1} v_{1} v_{s} u_{s+1} P u_{l+1} u_{x}$ is a heterochromatic path of length $l+1$, a contradiction to the choice of $P$.

Case 3.1.2 $k \equiv 2(\bmod 5)$. So, we have $s=2 l-k-3$ or $s=2 l-k-2$.

Case 3.1.2.1 If $s=2 l-k-3$, then $t_{1}=k-l-2 s+3=k-l-4 l+2 k+6+3=$ $3 k-5 l+6+3=5$, and so $s-2 \geq t_{2} \geq k-(l+s-1)-t_{1}=s+(k-l-2 s+1)-5=$ $s+(k-l-4 l+2 k+7-5)=s+(3 k-5 l+2)=s-2$. Then $x_{j_{0}-2}=s+1+2\left(j_{0}-3\right)=s+2 j_{0}-5$, $x_{j_{0}}=l-s+1-2\left(t_{1}-j_{0}\right)=l-s-2 t_{1}+2 j_{0}+1=l-s+2 j_{0}-9$ and $x^{\prime}=x_{j_{0}-2}=x_{j}-s-1$. Hence, $4 \leq x_{j_{0}}-x_{j_{0}-2}=s+1$, which implies $s \geq 3$. Since $s \leq k_{0} \leq 2 l-k=s+3=x_{1}+2$ and $k_{0}-s+1 \leq 4 \leq s+1$, we have $k_{0}<x_{1}=s+1$ by Lemma 3.3, then $k_{0}=s$.

If there exists an $x$ with $1 \leq x \leq x_{1}-1$ such that $u_{x} u_{l+1}$ has a color not in $\left\{i_{k_{0}}, i_{k_{0}+1}, \ldots, i_{l}, i_{l+1}, \ldots, i_{l+s-1}, i_{l+s}\right\}$, let $P^{\prime}=v_{1} P v_{s} u_{s+1} P u_{l+1} u_{x}$, then $P^{\prime}$ is a heterochromatic path of length $l+1$, a contradiction to the choice of $P$. 
Otherwise, there are at least $s-\left((l-1)-\left(k-l+k_{0}-1\right)\right)=s-2 l+k+k_{0}=s-s-3+s=$ $s-3$ colors in $\left\{i_{l+1}, i_{l+2}, \ldots, i_{l+s-1}\right\}$ that belong to $C\left(\left\{u_{1} u_{l+1}, u_{2} u_{l+1}, \ldots, u_{s} u_{l+1}\right\}\right)$.

If $s \geq 4$, then there exists an $x$ with $1 \leq x \leq s$ such that $u_{x} u_{l+1}$ has a color in $\left\{i_{l+1}, i_{l+s-2}, i_{l+s-1}\right\}$. Let

$$
\begin{aligned}
& P_{1}= \begin{cases}v_{2} P v_{s} & \text { if } u_{x} u_{l+1} \text { has color } i_{l+1} ; \\
v_{s-2} P^{-1} v_{1} v_{s} & \text { if } u_{x} u_{l+1} \text { has color } i_{l+s-2} \text { or } i_{l+s-1} .\end{cases} \\
& P^{\prime}= \begin{cases}P_{1} u_{s+1} P u_{l+1} u_{x} u_{x+1} & \text { if } 1 \leq x \leq s-1 \\
P_{1} u_{s+1} P u_{l+1} u_{s} u_{s-1} & \text { if } x=s\end{cases}
\end{aligned}
$$

Then, $P^{\prime}$ is a heterochromatic path of length $l+1$, a contradiction to the choice of $P$.

If $s=3$, then there are at least $x_{2}-1-1-\left((l-1)-\left(k-l+k_{0}-1\right)\right)=x_{2}-2 l+$ $k+k_{0}-2 \geq s+3-s-3+s-2=s-2$ colors in $\left\{i_{l+1}, \ldots, i_{l+s-1}\right\}$ that belong to $C\left(\left\{u_{1} u_{l+1}, \ldots, u_{x_{2}-1} u_{l+2}\right\}\right)$. So, there exists an edge $u_{x} u_{l+1}\left(1 \leq x \leq x_{2}-1\right)$ such that $u_{x} u_{l+1}$ has a color $i_{l+s-2}$ or $i_{l+s-1}$. Let

$$
P^{\prime}= \begin{cases}v_{1} v_{s} u_{s+1} P u_{l+1} u_{x} u_{x-1} & \text { if } 2 \leq x \leq s \\ v_{1} v_{s} u_{s+1} P u_{l+1} u_{1} u_{2} & \text { if } x=1 \\ v_{1} v_{s} u_{x_{2}} P u_{l+1} u_{x} P^{-1} u_{x-\left(x_{2}-s\right)} & \text { if } s+1 \leq x \leq x_{2}-1\end{cases}
$$

If $s+1 \leq x \leq x_{2}-1$, then $x-\left(x_{2}-s\right) \geq 2 s-x_{2}+1 \geq 2 s-\left(l-s+1-2\left(t_{1}-2\right)\right)+1=$ $3 s-l+6=6 l-3 k-9-l+6=5 l-3 k-3=5\left\lceil\frac{3 k}{5}\right\rceil-3 k-3=1$. So, $P^{\prime}$ is a heterochromatic path of length $l+1$, a contradiction to the choice of $P$.

Case 3.1.2.2 If $s=2 l-k-2$, then $t_{1}=k-l-2 s+3=k-l-4 l+2 k+4+3=$ $3 k-5 l+7=3, s-2 \geq t_{2} \geq k-(l+s-1)-t_{1}=s+(k-l-2 s+1)-3=s+(k-l-4 l+2 k+2)=$ $s+(3 k-5 l+2)=s-2$, and so $t_{2}=s-2$. Then, $j_{0}=t_{1}=3$ and $s+1 \leq x_{3}-x_{1} \leq s+2$ which implies $s \geq 2$. So, we have that $x_{1}=s+1$ and $x_{3}=x_{1}+s+2=2 s+3$ or $x_{3}=x_{1}+s+1=2 s+2$; or $x_{1}=s+2$ and $x_{3}=x_{1}+s+1=2 s+3$.

We first consider the case when $x_{1}=s+1$. Since $s \leq k_{0} \leq 2 l-k=s+2=x_{1}+1$ and $k_{0}-s+1 \leq 3 \leq s+1$, we have that $k_{0}<x_{1}=s+1$ by Lemma 3.3, and so $k_{0}=s$. If there exists an $x$ with $1 \leq x \leq s$ such that $u_{x} u_{l+1}$ has a color not in $\left\{i_{k_{0}}, i_{k_{0}+1}, \ldots, i_{l}, i_{l+1}, \ldots, i_{l+s-1}\right\}$, let $P^{\prime}=v_{1} P v_{s} u_{s+1} P u_{l+1} u_{x}$, then $P^{\prime}$ is a heterochromatic path of length $l+1$, a contradiction to the choice of $P$. So, there are at least $s-\left((l-1)-\left(k-l+k_{0}-1\right)\right)=s-2 l+k+k_{0}=s-s-2+k_{0}=s-2$ colors in $\left\{i_{l+1}, \ldots, i_{l+s-1}\right\}$ that belong to $C\left(\left\{u_{1} u_{l+1}, \ldots, u_{s} u_{l+1}\right\}\right)$.

If $s \geq 3$, then there is an edge $u_{x} u_{l+1}(1 \leq x \leq s)$ such that $u_{x} u_{l+1}$ has a color in $\left\{i_{l+s-2}, i_{l+s-1}\right\}$. Let

$$
P^{\prime}= \begin{cases}v_{s-2} P^{-1} v_{1} u_{s+1} P u_{l+1} u_{x} u_{x-1} & \text { if } 2 \leq x \leq s \\ v_{s-2} P^{-1} v_{1} u_{s+1} P u_{l+1} u_{1} u_{2} & \text { if } x=1\end{cases}
$$

Then, $P^{\prime}$ is a heterochromatic path of length $l+1$, a contradiction to the choice of $P$.

If $s=2$, then we have $4 \leq x_{3}-x_{1} \leq s+2=4$ which implies that $x_{1}=x_{3}-4, x^{\prime}=x_{1}$ or $x_{1}+1$. There are at least $x_{2}-1-\left((l-1)-\left(k-l+k_{0}-1\right)\right)-1=x_{2}-2 l+k+k_{0}-2=$ $x_{2}-s-2+k_{0}-2 \geq s+3-s-2+s-2=s-1$ colors in $\left\{i_{l+1}, \ldots, i_{l+s-1}\right\}$ that belong to 
$C\left(\left\{u_{1} u_{l+1}, \ldots, u_{t_{2}-1} u_{l+1}\right\}\right)$. So, there is some $1 \leq x \leq x_{2}-1$ such that $u_{x} u_{l+1}$ has color $i_{l+1}$. If $x^{\prime}=x_{1}=s+1$, then $x \neq s+1$. Let

$$
P^{\prime}= \begin{cases}v_{s} u_{s+1} P u_{l+1} u_{x} u_{x-1} & \text { if } x=2 ; \\ v_{s} u_{s+1} P u_{l+1} u_{1} u_{2} & \text { if } x=1 ; \\ v_{s} u_{x_{2}} P u_{l+1} u_{x} P^{-1} u_{x-\left(x_{2}-s\right)} & \text { if } s+2 \leq x \leq x_{2}-1 .\end{cases}
$$

Note that if $s+2 \leq x \leq x_{2}-1$, then $x-\left(x_{2}-s\right)=x+s-x_{2} \geq 2 s+2-x_{2}=2 s+2-x_{1}-2=$ $2 s-x_{1}=s-1=1$, and so $P^{\prime}$ is a heterochromatic path of length $l+1$, a contradiction to the choice of $P$. If $x^{\prime}=x_{1}+1=s+2$, let $P^{\prime}=u_{1} P u_{x_{1}} v_{s} u_{x_{3}} P u_{l+1} u_{x_{1}+1} P u_{x_{3}-1}$, then $P^{\prime}$ is a heterochromatic path of length $l+1$.

Now we consider the case when $x^{\prime}=x_{1}=s+2$ and $x_{3}=2 s+3$. Since $s+1=$ $x_{3}-x_{1} \geq 4$, we have $s \geq 3$. Then $s \leq k_{0} \leq 2 l-k=s+2=x_{1}$ and $k_{0}-s+1 \leq 3 \leq s$, and so $s \leq k_{0} \leq x_{1}-1=s+1$.

If there exists an $2 \leq x \leq s$ such that $u_{x} u_{l+1}$ has a color not in $\left\{i_{k_{0}}, i_{k_{0}+1}, \ldots, i_{l}\right.$, $\left.i_{l+1}, \ldots, i_{l+s-1}\right\}$, let

$$
P^{\prime}= \begin{cases}v_{1} P v_{s} u_{s+2} P u_{l+1} u_{x} u_{x-1} & \text { if } u_{x} u_{l+1} \text { has a color different from } i_{x-1} ; \\ v_{1} P v_{s} u_{s+2} P u_{l+1} u_{x} u_{x+1} & \text { if } u_{x} u_{l+1} \text { has color } i_{x-1},\end{cases}
$$

then $P^{\prime}$ is a heterochromatic path of length $l+1$, a contradiction to the choice of $P$.

Otherwise, there are at least $(s-1)-\left((l-1)-\left(k-l+k_{0}-1\right)\right)=s-2 l+k+$ $k_{0}-1=s-s-2+k_{0}-1=k_{0}-3 \geq s-3$ colors in $\left\{i_{l+1}, \ldots, i_{l+s-1}\right\}$ that belong to $C\left(\left\{u_{2} u_{l+1}, \ldots, u_{s} u_{l+1}\right\}\right)$.

If $s \geq 4$, then there is an $2 \leq x \leq s$ such that $u_{x} u_{l+1}$ has color $i_{l+1}, i_{l+s-2}$ or $i_{l+s-1}$. Let

$$
P_{1}= \begin{cases}v_{2} P v_{s} & \text { if } u_{x} u_{l+1} \text { has color } i_{l+1} ; \\ v_{s-2} P^{-1} v_{1} v_{s} & \text { if } u_{x} u_{l+1} \text { has color } i_{l+s-2} \text { or } i_{l+s-1} . \\ P_{1} u_{s+2} P u_{l+1} u_{x} u_{x-1} u_{x-2} & \text { if } 3 \leq x \leq s ; \\ P_{1} u_{s+2} P u_{l+1} u_{2} u_{3} u_{4} & \text { if } x=2 .\end{cases}
$$

Then, $P^{\prime}$ is a heterochromatic path of length $l+1$, a contradiction to the choice of $P$.

If $s=3$ and $k_{0}=s+1=4$, then there are at least $k_{0}-3=1$ color of $\left\{i_{l+1}, i_{l+2}=i_{l+s-1}\right\}$ that belong to $C\left(\left\{u_{2} u_{l+1}, u_{3} u_{l+1}\right\}\right)$. Let

$$
P^{\prime}= \begin{cases}v_{2} v_{3} u_{5} P u_{l+1} u_{2} u_{3} u_{4} & \text { if } u_{2} u_{l+1} \text { has color } i_{l+1} ; \\ v_{2} v_{3} u_{5} P u_{l+1} u_{3} u_{2} u_{1} & \text { if } u_{3} u_{l+1} \text { has color } i_{l+1} ; \\ v_{2} v_{1} v_{3} u_{5} P u_{l+1} u_{x} u_{x-1} & \text { if } u_{x} u_{l+1}(x=2,3) \text { has color } i_{l+2} .\end{cases}
$$

Then, $P^{\prime}$ is a heterochromatic path of length $l+1$, a contradiction to the choice of $P$.

If $s=3$ and $k_{0}=s=3$, then $x_{1}=s+2=5$ and $x_{3}=2 s+3=9$, and so $x_{2}=7$, and then $x^{\prime}=x_{1}=5$. There are at least $k_{0}-3+x_{2}-x_{1}-1=x_{2}-x_{1}-1=1$ colors in $\left\{i_{l+1}, i_{l+2}=i_{l+s-1}\right\}$ that belong to $C\left(\left\{u_{2} u_{l+1}, u_{3} u_{l+1}, u_{6} u_{l+1}\right\}\right)$. Let

$$
P^{\prime}=\left\{\begin{array}{ll}
v_{2} v_{3} u_{5} P u_{l+1} u_{2} u_{3} u_{4} & \text { if } u_{2} u_{l+1} \text { has color } i_{l+1} ; \\
v_{2} v_{3} u_{5} P u_{l+1} u_{3} u_{2} u_{1} & \text { if } u_{3} u_{l+1} \text { has color } i_{l+1} ; \\
v_{2} v_{3} u_{7} P u_{l+1} u_{6} P^{-1} u_{2} & \text { if } u_{6} u_{l+1} \text { has color } i_{l+1} ; \\
v_{2} v_{1} v_{3} u_{5} P u_{l+1} u_{x} u_{x-1} & \text { if } u_{x} u_{l+1}(x=2,3) \text { has color } i_{l+2} \\
v_{2} v_{1} v_{3} u_{7} P u_{l+1} u_{6} P^{-1} u_{3} & \text { if } u_{6} u_{l+1} \text { has color } i_{l+2} .
\end{array} ;\right.
$$


Then, $P^{\prime}$ is a heterochromatic path of length $l+1$, a contradiction to the choice of $P$.

Case 3.1.3 $k \equiv 4(\bmod 5)$ and $s=2 l-k-2$. Then, $t_{1}=k-l-2 s+3=$ $k-l-4 l+2 k+4+3=3 k-5 l+7=4, s-2 \geq t_{2} \geq k-(l+s-1)-t_{1}=$ $s+(k-l-2 s+1)-4=s+(k-l-4 l+2 k+1)=s+(3 k-5 l+1)=s-2$ which implies $t_{2}=s-2, x_{j_{0}-2}=s+1+2\left(j_{0}-3\right)=s+2 j_{0}-5, x_{j_{0}}=l-s+1-2\left(t_{1}-j_{0}\right)=l-s+2 j_{0}-7$ and $x_{j_{0}-2}=x_{j_{0}}-s-1$. So, $6 \leq x_{4}-x_{1}=l-s+1-s-1=l-2 s=s+(l-3 s)=$ $s+(l-6 l+3 k+6)=s+(3 k-5 l+6)=s+3$ which implies that $s \geq 3$. On the other hand, $s \leq k_{0} \leq 2 l-k=s+2=x_{1}+1$ and $k_{0}-s+1 \leq 3 \leq s$, and then $k_{0}<x_{1}$, and so $k_{0}=s$.

If there is an $1 \leq x \leq s$ such that $u_{x} u_{l+1}$ has a color not in $\left\{i_{k_{0}}, i_{k_{0}+1}, \ldots, i_{l}, i_{l+1}\right.$, $\left.\ldots, i_{l+s-1}, i_{l+s}\right\}$, let $P^{\prime}=v_{1} P v_{s} u_{s+1} P u_{l+1} u_{x}$, then $P^{\prime}$ is a heterochromatic path of length $l+1$, a contradiction to the choice of $P$. Otherwise, there are at least $s-((l-1)-(k-$ $\left.\left.l+k_{0}-1\right)\right)=s-2 l+k+k_{0}=s-s-2+s=s-2$ colors in $\left\{i_{l+1}, i_{l+2}, \ldots, i_{l+s-1}\right\}$ that belong to $C\left(\left\{u_{1} u_{l+1}, u_{2} u_{l+1}, \ldots, u_{s} u_{l+1}\right\}\right)$. In other words, there is some $1 \leq x \leq s$ such that $u_{x} u_{l+1}$ has color $i_{l+s-2}$ or $i_{l+s-1}$. Let

$$
P^{\prime}= \begin{cases}v_{s-2} P^{-1} v_{1} v_{s} u_{s+1} P u_{l+1} u_{x} u_{x-1} & \text { if } 2 \leq x \leq s \\ v_{s-2} P^{-1} v_{1} v_{s} u_{s+1} P u_{l+1} u_{1} u_{2} & \text { if } x=1\end{cases}
$$

Then, $P^{\prime}$ is a heterochromatic path of length $l+1$, a contradiction to the choice of $P$.

Case $3.2 C\left(u_{x^{\prime}} u_{l+1}\right) \notin\left\{i_{1}, i_{2}, \ldots, i_{l}, i_{l+1}, \ldots, i_{l+s-1}, i_{l+s+j_{0}-2}, i_{l+s+j_{0}-1}\right\}$. Then $x_{j_{0}-1}$ $\leq x^{\prime} \leq x_{j_{0}}-1$. Let $P^{\prime}=v_{1} P v_{s} u_{x_{0}} P u_{l+1} u_{x^{\prime}} P^{-1} u_{x^{\prime}-\left(x_{j_{0}}-s\right)+1}$. Note that

$$
\begin{aligned}
& x^{\prime}-\left(x_{j_{0}}-s\right)+1 \geq x_{j_{0}-1}-x_{j_{0}}+s+1 \\
& \geq\left(s+1+2\left(j_{0}-2\right)\right)-\left(l-s+1-2\left(t_{1}-j_{0}\right)\right)+s+1 \\
& =3 s-l+2 t_{1}-3 \\
& \geq 3 s-l+2 \max \{k-l-2 s+3,3\}-3 \\
& = \begin{cases}2 k-3 l-s+3 & \text { if } s \leq 2 l-k-2 \text { and } k \equiv 2,4(\bmod 5) ; \\
2 k-3 l-s+3 & \text { if } s \leq 2 l-k-1 \text { and } k \equiv 1(\bmod 5) ; \\
3 s-l+3 & \text { if } s=2 l-k \text { or } s=2 l-k-1 \text { and } k \equiv 2,4(\bmod 5) ; \\
3 s-l+3 & \text { if } s=2 l-k \text { and } k \equiv 1(\bmod 5) .\end{cases}
\end{aligned}
$$

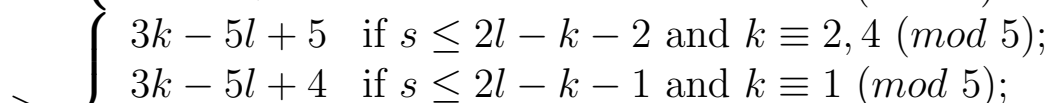

$$
\begin{aligned}
& \geq \begin{cases}3 k-5 l+4 & \text { if } s \leq 2 l-k-1 \text { and } k \equiv 1(\bmod 5) \\
5 l-3 k & \text { if } s=2 l-k \text { or } s=2 l-k-1 \text { and } k \equiv 2,4(\bmod 5) ; \\
5 l-3 k+3 & \text { if } s=2 l-k \text { and }\end{cases} \\
& \geq 1
\end{aligned}
$$

So, $P^{\prime}$ is a heterochromatic path of length $l+1$, a contradiction to the choice of $P$.

Lemma 3.10 Let $k \geq 8$ and $k \equiv 1,2,4(\bmod 5), t_{1} \geq 2, C\left(\left\{u_{1} u_{l+1}, \ldots, u_{x_{t_{1}}-1} u_{l+1}\right\}\right)$ $\subseteq\left\{i_{1}, i_{2}, \ldots, i_{l+s-1}, i_{l+s+t_{1}-1}\right\}$. Then there is no $x_{1} \leq x^{\prime} \leq x_{t_{1}}-1$ such that $C\left(u_{x^{\prime}} u_{l+1}\right) \in$ $\left\{i_{l+1}, \ldots, i_{l+s-x_{t_{1}}+x_{1}+2 t_{1}-4}\right\} \cup\left\{i_{l+\left\lceil\frac{s-t_{2}-1}{2}\right\rceil+x_{t_{1}}-x_{1}-2 t_{1}+2}, \ldots i_{l+s-1}\right\}$.

Proof. Since $C\left(\left\{u_{1} u_{l+1}, u_{2} u_{l+1}, \ldots, u_{x_{t_{1}}-1} u_{l+1}\right\}\right) \subseteq\left\{i_{1}, i_{2}, \ldots, i_{l+s-1}, i_{l+s+t_{1}-1}\right\}$, we have that $k-(l+s) \leq l-1-x_{t_{1}}+1$ which implies that $x_{t_{1}} \leq 2 l-k+s$, and $x_{1}+2\left(t_{1}-1\right) \leq x_{t_{1}}$ 
which implies that $2 t_{1} \leq x_{t_{1}}-x_{1}+2 \leq 2 l-k+s-s-1+2=2 l-k+1$.

If there exists an $x_{1} \leq x^{\prime} \leq x_{t_{1}}-1$ such that $u_{x^{\prime}} u_{l+1}$ has a color in $\left\{i_{l+1}, \ldots\right.$, $\left.i_{l+s-x_{t_{1}}+x_{1}+2 t_{1}-4}\right\}$, then there exists a $2 \leq j_{0} \leq t_{1}$ such that $x_{j_{0}-1} \leq x^{\prime} \leq x_{j_{0}}-1$. Let $P^{\prime}=v_{s-x_{t_{1}}+x_{1}+2 t_{1}-3} P v_{s} u_{x_{j_{0}}} P u_{l+1} u_{x^{\prime}} P^{-1} u_{x^{\prime}-\left(x_{j_{0}}-x_{t_{1}}+x_{1}+2 t_{1}-4\right)+1}$. Since $k-l-2 s+3 \geq$ $k-l-4 l+2 k+3=3 k-5 l+3=3 k-5\left\lceil\frac{3 k}{5}\right\rceil+3$, we have that $k-l-2 s+3<2$ if and only if $s=2 l-k$ when $k \equiv 1,2,4(\bmod 5)$, or $s=2 l-k-1$ when $k \equiv 2(\bmod 5)$. So,

$$
\begin{aligned}
& s-x_{t_{1}}+x_{1}+2 t_{1}-3 \\
& \geq s-2 l+k-s+s+1+2 \max \{k-l-2 s+3,2\}-3 \\
& =s-2 l+k+2 \max \{k-l-2 s+3,2\}-2 \\
& = \begin{cases}3 k-4 l-3 s+4 & \text { if } s \leq 2 l-k-1 \text { and } k \equiv 1,4(\bmod 5) ; \\
3 k-4 l-3 s+4 & \text { if } s \leq 2 l-k-2 \text { and } k \equiv 2(\bmod 5) ; \\
k-2 l+s+2 & \text { if } s=2 l-k \text { and } k \equiv 1,4(\bmod 5) ; \\
k-2 l+s+2 & \text { if } s=2 l-k \text { or } s=2 l-k-1 \text { and } k \equiv 2(\bmod 5) .\end{cases} \\
& \geq \begin{cases}6 k-10 l+7 & \text { if } s \leq 2 l-k-1 \text { and } k \equiv 1,4(\bmod 5) \\
6 k-10 l+10 & \text { if } s \leq 2 l-k-2 \text { and } k \equiv 2(\bmod 5) \\
2 & \text { if } s=2 l-k \text { and } k=1,4(\bmod 5)\end{cases} \\
& \geq \begin{cases}2 & \text { if } s=2 l-k \text { and } k \equiv 1,4(\bmod 5) \\
1 & \text { if } s=2 l-k \text { or } s=2 l-k-1 \text { and } k \equiv 2(\bmod 5),\end{cases} \\
& \geq 1
\end{aligned}
$$

and $x^{\prime}-\left(x_{j_{0}}-x_{t_{1}}+x_{1}+2 t_{1}-4\right)+1 \geq x_{j_{0}-1}-x_{j_{0}}+x_{t_{1}}-x_{1}-2 t_{1}+5=\left(x_{j_{0}-1}-x_{1}\right)+$ $\left(x_{t_{1}}-x_{j_{0}}\right)-2 t_{1}+5 \geq 2\left(j_{0}-2\right)+2\left(t_{1}-j_{0}\right)-2 t_{1}+5=1$. Then, $P^{\prime}$ is a heterochromatic path of length $l+1$, a contradiction to the choice of $P$.

Next we consider the following two cases:

Case $1 s-x_{t_{1}}+x_{1}+2 t_{1}-4 \leq\left\lceil\frac{s-t_{2}-1}{2}\right\rceil+x_{t_{1}}-x_{1}-2 t_{1}+1$.

If there exists an $x_{1} \leq x^{\prime} \leq x_{t_{1}}-1$ such that $u_{x^{\prime}} u_{l+1}$ has a color in $\left\{i_{l+\left\lceil\frac{s-t_{2}-1}{2}\right\rceil+x_{t_{1}}-x_{1}-2 t_{1}+2}, \ldots, i_{l+s-1}\right\}$, then there exists a $2 \leq j_{0} \leq t_{1}$ such that $x_{j_{0}-1} \leq$ $x^{\prime} \leq x_{j_{0}}-1$. Since

$$
\begin{aligned}
& t_{2}-\left[(s-2)-\left(\left\lceil\frac{s-t_{2}-1}{2}\right\rceil+x_{t_{1}}-x_{1}-2 t_{1}+2\right)\right] \\
& \geq t_{2}-s+2+s-x_{t_{1}}+x_{1}+2 t_{1}-3 \\
& =t_{2}-x_{t_{1}}+x_{1}+2 t_{1}-1 \\
& \geq k-l-s+1-t_{1}-(l-s+1)+(s+1)+2 t_{1}-1 \\
& =k-2 l+s+t_{1} \\
& \geq k-2 l+s+\max \{k-l-2 s+3,2\} \\
& \left\{\begin{array}{l}
2 k-3 l-s+3 \text { if } s \leq 2 l-k-1 \text { and } k \equiv 1,4(\bmod 5) \\
2 k-3 l-s+3 \text { if } s \leq 2 l-k-2 \text { and } k \equiv 2(\bmod 5)
\end{array}\right.
\end{aligned}
$$

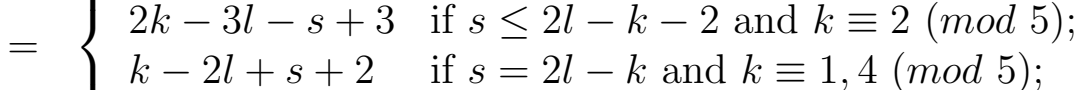

$$
\begin{aligned}
& k-2 l+s+2 \text { if } s=2 l-k \text { or } s=2 l-k-1 \text { and } k \equiv 2(\bmod 5) \text {. } \\
& \geq \begin{cases}3 k-5 l+4 & \text { if } s \leq 2 l-k-1 \text { and } k \equiv 1,4(\bmod 5) \\
3 k-5 l+5 & \text { if } s \leq 2 l-k-2 \text { and } k \equiv 2(\bmod 5) \\
2 & \text { if } s=2 l-k \text { and } k \equiv 1,4(\bmod 5) \\
1 & \text { if } s=2 l-k \text { or } s=2 l-k-1 \text { and } k \equiv 2(\bmod 5) .\end{cases} \\
& \geq 1
\end{aligned}
$$


and $y_{t_{2}-\left[(s-2)-\left(\left\lceil\frac{s-t_{2}-1}{2}\right\rceil+x_{t_{1}}-x_{1}-2 t_{1}+2\right)\right]} \leq\left\lceil\frac{s-t_{2}-1}{2}\right\rceil+x_{t_{1}}-x-1-2 t_{1}+2$, there exists a $y^{\prime} \in\left\{1,2, \ldots,\left\lceil\frac{s-t_{2}-1}{2}\right\rceil\right\} \cup\left\{\left\lceil\frac{s-t_{2}-1}{2}\right\rceil+x_{t_{1}}-x_{1}-2 t_{1}+2, \ldots, x_{t_{1}}-x_{1}-2 t_{1}+\left\lceil\frac{s-t_{2}-1}{2}\right\rceil-\right.$ $\left.\left\lfloor\frac{s-t_{2}-1}{2}\right\rfloor+3\right\}$ such that $y^{\prime} \in\left\{y_{1}, y_{2}, \ldots, y_{t_{2}}\right\}$. If $y^{\prime} \in\left\{1,2, \ldots,\left\lceil\frac{s-t_{2}-1}{2}\right\rceil\right\}$, then let $P^{\prime}=$ $v_{y^{\prime}+\left(x_{t_{1}}-x_{1}-2 t_{1}+2\right)} P^{-1} v_{y^{\prime}} v_{s} u_{x_{j_{0}}} P u_{l+1} u_{x^{\prime}} P^{-1} u_{x^{\prime}-\left(x_{j_{0}}-x_{t_{1}}+x_{1}+2 t_{1}-4\right)+1}$. Since $y^{\prime}+\left(x_{t_{1}}-x_{1}-\right.$ $\left.2 t_{1}+2\right) \leq\left\lceil\frac{s-t_{2}-1}{2}\right\rceil+x_{t_{1}}-x_{1}-2 t_{1}+2$, and $x^{\prime}-\left(x_{j_{0}}-x_{t_{1}}+x_{1}+2 t_{1}-4\right)+1 \geq x_{j_{0}-1}-x_{j_{0}}+$ $x_{t_{1}}-x_{1}-2 t_{1}+5=\left(x_{j_{0}-1}-x_{1}\right)+\left(x_{t_{1}}-x_{j_{0}}\right)-2 t_{1}+s \geq 2\left(j_{0}-2\right)+2\left(t_{1}-j_{0}\right)-2 t_{1}+5=1$, $P^{\prime}$ is a heterochromatic path of length $l+1$, a contradiction to the choice of $P$. Otherwise, if $y^{\prime} \in\left\{\left\lceil\frac{s-t_{2}-1}{2}\right\rceil+x_{t_{1}}-x_{1}-2 t_{1}+2, \ldots, x_{t_{1}}-x_{1}-2 t_{1}+\left\lceil\frac{s-t_{2}-1}{2}\right\rceil-\left\lfloor\frac{s-t_{2}-1}{2}\right\rfloor+3\right\}$, then let $P^{\prime}=v_{y^{\prime}-\left(x_{t_{1}}-x_{1}-2 t_{1}+2\right)} P v_{y^{\prime}} v_{s} u_{x_{j_{0}}} P u_{l+1} u_{x^{\prime}} P^{-1} u_{x^{\prime}-\left(x_{j_{0}}-x_{t_{1}}+x_{1}+2 t_{1}-4\right)+1}$. Since $y^{\prime}-\left(x_{t_{1}}-\right.$ $\left.x_{1}-2 t_{1}+2\right) \geq x_{t_{1}}-x_{1}-2 t_{1}+\left\lceil\frac{s-t_{2}-1}{2}\right\rceil-\left\lfloor\frac{s-t_{2}-1}{2}\right\rfloor+3-x_{t_{1}}+x_{1}+2 t_{1}-2 \geq 1, P^{\prime}$ is a heterochromatic path of length $l+1$, a contradiction to the choice of $P$.

Case $2 s-x_{t_{1}}+x_{1}+2 t_{1}-4 \geq\left\lceil\frac{s-t_{2}-1}{2}\right\rceil+x_{t_{1}}-x_{1}-2 t_{1}+2$.

If there exists an $x_{1} \leq x^{\prime} \leq x_{t_{1}}-1$ such that $u_{x^{\prime}} u_{l+1}$ has a color in $\left\{l+s-x_{t_{1}}+x_{1}+\right.$ $\left.2 t_{1}-3, l+s-x_{t_{1}}+x_{1}+2 t_{1}-2, \ldots, l+s-1\right\}$, then there exists a $2 \leq j_{0} \leq t_{1}$ such that $x_{j_{0}-1} \leq x^{\prime} \leq x_{j_{0}}-1$. Since

$$
\begin{aligned}
& t_{2}-\left[(s-2)-\left(s-x_{t_{1}}+x_{1}+2 t_{1}-3\right)\right] \\
= & t_{2}-x_{t_{1}}+x_{1}+2 t_{1}-1 \\
\geq & \left(k-l-s+1-t_{1}\right)-(l-s+1)+(s+1)+2 t_{1}-1 \\
= & k-2 l+s+t_{1} \\
\geq & k-2 l+s+\max \{k-l-2 s+3,2\} \\
\geq & 1
\end{aligned}
$$

and $y_{t_{2}-\left[(s-2)-\left(s-x_{t_{1}}+x_{1}+2 t_{1}-3\right)\right]} \leq s-x_{t_{1}}+x_{1}+2 t_{1}-3$, there exists a $y^{\prime} \in\{1,2, \ldots$, $\left.\left\lceil\frac{s-t_{2}-1}{2}\right\rceil\right\} \cup\left\{s-x_{t_{1}}+x_{1}+2 t_{1}-3, \ldots, s-x_{t_{1}}+x_{1}+2 t_{1}-\left\lfloor\frac{s-t_{2}-1}{2}\right\rfloor-2\right\}$ such that $y^{\prime} \in$ $\left\{y_{1}, y_{2}, \ldots, y_{t_{2}}\right\}$. If $y^{\prime} \in\left\{1,2, \ldots,\left\lceil\frac{s-t_{2}-1}{2}\right\rceil\right\}$, then let $P^{\prime}=v_{y^{\prime}+\left(x_{t_{1}}-x_{1}-2 t_{1}+2\right)} P^{-1} v_{y^{\prime}} v_{s} u_{x_{j_{0}}}$ $P u_{l+1} u_{x^{\prime}} P^{-1} u_{x^{\prime}-\left(x_{j_{0}}-x_{t_{1}}+x_{1}+2 t_{1}-4\right)+1}$. Since $y^{\prime}+\left(x_{t_{1}}-x_{1}-2 t_{1}+2\right) \leq\left\lceil\frac{s-t_{2}-1}{2}\right\rceil+x_{t_{1}}-$ $x_{1}-2 t_{1}+2 \leq s-x_{t_{1}}+x_{1}+2 t_{1}-4=\left(s-x_{t_{1}}+x_{1}+2 t_{1}-3\right)-1, P^{\prime}$ is a heterochromatic path of length $l+1$, a contradiction to the choice of $P$. Otherwise, if $y^{\prime} \in\left\{s-x_{t_{1}}+x_{1}+2 t_{1}-3, \ldots, s-x_{t_{1}}+x_{1}+2 t_{1}-\left\lfloor\frac{s-t_{2}-1}{2}\right\rfloor-2\right\}$, then let $P^{\prime}=$ $v_{y^{\prime}-\left(x_{t_{1}}-x_{1}-2 t_{1}+2\right)} P v_{y^{\prime}} v_{s} u_{x_{j_{0}}} P u_{l+1} u_{x^{\prime}} P^{-1} u_{x^{\prime}-\left(x_{j_{0}}-x_{t_{1}}+x_{1}+2 t_{1}-4\right)+1}$. Since $y^{\prime}-\left(x_{t_{1}}-x_{1}-2 t_{1}+\right.$ 2) $\geq s-x_{t_{1}}+2 t_{1}-2-\left\lfloor\frac{s-t_{2}-1}{2}\right\rfloor-\left(x_{t_{1}}-x_{1}-2 t_{1}+2\right) \geq\left\lceil\frac{s-t_{2}-1}{2}\right\rceil+x_{t_{1}}-x_{1}-2 t_{1}+4-$ $\left\lfloor\frac{s-t_{2}-1}{2}\right\rfloor-\left(x_{t_{1}}-x_{1}-2 t_{1}+2\right)=\left\lceil\frac{s-t_{2}-1}{2}\right\rceil-\left\lfloor\frac{s-t_{2}-1}{2}\right\rfloor+2 \geq 2, P^{\prime}$ is a heterochromatic path of length $l+1$, a contradiction to the choice of $P$.

Lemma 3.11 Let $k \geq 8, k \equiv 1,2,4(\bmod 5)$ and $t_{1}=0$. Then $i_{1} \notin C\left(\left\{u_{1} v_{s}, u_{2} v_{s}, \ldots\right.\right.$, $\left.\left.u_{l} v_{s}\right\}\right)$.

Proof. Since $t_{1}=0$, we have that $k_{0}=s=2 l-k, k \equiv 2,4(\bmod 5), t_{2}=s-2$ if $k \equiv 4(\bmod 5)$ and $t_{2} \geq s-3$ if $k \equiv 2(\bmod 5)$, and $\mid C\left(\left\{u_{1} u_{l+1}, \ldots, u_{l-1} u_{l+1}\right\}\right)-$ $\left\{i_{k_{0}}, i_{k_{0}+1}, \ldots, i_{l}\right\} \mid=l-1$ by Lemma 3.5 .

If $i_{1} \in C\left(\left\{u_{1} v_{s}, u_{2} v_{s}, \ldots, u_{l} v_{s}\right\}\right)$, then by Lemma 3.5 we have $i_{1} \in C\left(\left\{u_{s+1} v_{s}, \ldots\right.\right.$, $\left.\left.u_{l} v_{s}\right\}\right)$. On the other hand, if there exists an $x \in\{l-s+3, l-s+4, \ldots, l\}$ such that $u_{x} u_{l+1}$ 
has color $i_{1}$, then let $P^{\prime}=v_{1} P v_{s} u_{x} P^{-1} u_{x-(l+2-s)+1}$. Note that when $l-s+3 \leq x \leq l$, we have that $x-(l+2-s)+1 \geq l-s+3-l-2+s+1=2$, and so $P^{\prime}$ is a heterochromatic path of length $l+1$, a contradiction to the choice of $P$. So, there exists an $s+1 \leq x \leq l-s+2$ such that $u_{x} u_{l+1}$ has color $i_{1}$.

If there exists an $2 \leq x^{\prime} \leq x-1$ such that $u_{x^{\prime}} u_{l+1}$ has a color not in $\left\{i_{1}, i_{2}, \ldots, i_{l+s-1}\right\}$, then let

$$
P^{\prime}= \begin{cases}v_{1} P v_{s} u_{x} P u_{l+1} u_{x^{\prime}} P^{-1} u_{x^{\prime}-(x-s)+1} & \text { if } x-s+1 \leq x^{\prime} \leq x-1 \\ v_{1} P v_{s} u_{x} P u_{l+1} u_{x^{\prime}} P u_{x^{\prime}+(x-s)-1} & \text { if } 2 \leq x^{\prime} \leq x-s\end{cases}
$$

Note that if $2 \leq x^{\prime} \leq x-s$, then $x^{\prime}+(x-s)-1 \leq x+(x-2 s-1) \leq x+(l-s+$ $2-2 s-1)=x+(l-3 s+1)=x+(l-6 l+3 k+1)=x+(3 k-5 l+1) \leq x-2$, and so $P^{\prime}$ is a heterochromatic path of length $l+1$, a contradiction to the choice of $P$. So, $C\left(\left\{u_{2} u_{l+1}, \ldots, u_{x-1} u_{l+1}\right\}\right) \subseteq\left\{i_{1}, i_{2}, \ldots, i_{l+s-1}\right\}$.

We distinguish the following two cases:

Case $1 x=s+1$.

If there exists an $1 \leq x^{\prime} \leq x-1$ such that $u_{x^{\prime}} u_{l+1}$ has a color in $\left\{i_{2}, \ldots, i_{k_{0}-1}\right\}$, then let $P^{\prime}=v_{1} P v_{s} u_{s+1} P u_{l+1} u_{x^{\prime}}$, which is a heterochromatic path of length $l+1$, a contradiction to the choice of $P$. So, there are $x-2-1=x-3=s-2$ colors in $\left\{i_{l+1}, \ldots, i_{l+s-1}\right\}$ that belong to $C\left(\left\{u_{2} u_{l+1}, \ldots, u_{x-1} u_{l+1}\right\}\right)$. Then, there exists an $2 \leq x^{\prime} \leq s$ such that $u_{x^{\prime}} u_{l+1}$ has color $i_{l+1}$ or $i_{l+s-1}$. Since $k \geq 8, s=2 l-k$ and $t_{2} \geq s-3$ if $s \equiv 2(\bmod 5)$ and $t_{2}=s-2$ if $s \equiv 4(\bmod 5)$, we have that $\{1,2\} \cap\left\{y_{1}, y_{2}, \ldots, y_{t_{2}}\right\} \neq \emptyset$. Let

$$
\begin{aligned}
& P_{1}= \begin{cases}v_{2} P v_{s} & \text { if } u_{x^{\prime}} u_{l+1} \text { has color } i_{l+1} \\
v_{s-2} P^{-1} v_{1} v_{s} & \text { if } 1 \in\left\{y_{1}, \ldots, y_{t_{2}}\right\} \text { and } u_{x^{\prime}} u_{l+1} \text { has color } i_{l+s-1} ; \\
v_{s-1} P^{-1} v_{2} v_{s} & \text { if } 2 \in\left\{y_{1}, \ldots, y_{t_{2}}\right\} \text { and } u_{x^{\prime}} u_{l+1} \text { has color } i_{l+s-1} .\end{cases} \\
& P^{\prime}= \begin{cases}P_{1} u_{s+1} P u_{l+1} u_{x^{\prime}} u_{x^{\prime}-1} & \text { if } 3 \leq x^{\prime} \leq s ; \\
P_{1} u_{s+1} P u_{l+1} u_{2} u_{3} & \text { if } x^{\prime}=2 ;\end{cases}
\end{aligned}
$$

Then, $P^{\prime}$ is a heterochromatic path of length $l+1$, a contradiction to the choice of $P$.

Case $2 x \geq s+2$.

There are at least $(x-2)-\left(k_{0}-1\right)=x-k_{0}-1$ different colors in $\left\{i_{l+1}, \ldots, i_{l+s-1}\right\}$ that belong to $C\left(\left\{u_{2} u_{l+1}, \ldots, u_{x-1} u_{l+1}\right\}\right)$. Since $\left|\left\{l+s-\left\lceil\frac{x}{2}\right\rceil+1, \ldots, l+\left\lceil\frac{x}{2}\right\rceil-2\right\}\right|=$ $2\left\lceil\frac{x}{2}\right\rceil-s-2 \leq x+1-s-2=x-k_{0}-1$, if there exists an $2 \leq x^{\prime} \leq x-1$ such that $u_{x^{\prime}} u_{l+1}$ has a color in $\left\{i_{l+1}, \ldots, i_{l+s-\left\lceil\frac{x}{2}\right\rceil}, i_{l+\left\lceil\frac{x}{2}\right\rceil-1}, \ldots, i_{l+s-1}\right\}$, then by Lemma 3.7 there is a heterochromatic path of length $l+1$, a contradiction to the choice of $P$. So, $\left\lceil\frac{x}{2}\right\rceil=\frac{x+1}{2}$, and since $l+s-\left\lceil\frac{x}{2}\right\rceil+1=l+1+\frac{2 s-x-1}{2} \geq l+1+\frac{2 s-l+s-3}{2}=l+1+\frac{5 l-3 k-3}{2} \geq l+1$, $l+\left\lceil\frac{x}{2}\right\rceil-2 \leq l+\frac{l-s+1}{2}-1=l+s-\frac{l-3 s-1}{2}=l+s-\frac{3 k-5 l-1}{2} \leq l+s-1$, there exists an $x^{\prime} \in\left\{2, \ldots,\left\lfloor\frac{x}{2}\right\rfloor,\left\lfloor\frac{x}{2}\right\rfloor+2, \ldots, x-1\right\}$ such that $u_{x^{\prime}} u_{l+1}$ has color $i_{l+s-\left\lceil\frac{x}{2}\right\rceil+1}$ or $i_{l+\left\lceil\frac{x}{2}\right\rceil-2}$. On the other hand, it is not hard to check that $\left\{1,\left\lceil\frac{x}{2}\right\rceil-2\right\} \cap\left\{y_{1}, \ldots, y_{t_{2}}\right\} \neq \emptyset$ since $t_{2} \geq s-3$ if $k \equiv 2(\bmod 5)$ and $t_{2}=s-2$ if $k \equiv 4(\bmod 5)$. Let

$$
P_{1}= \begin{cases}v_{s-\left\lceil\frac{x}{2}\right\rceil+2} P v_{s} & \text { if } u_{x^{\prime}} u_{l+1} \text { has color } i_{l+s-\left\lceil\frac{x}{2}\right\rceil+1} \\ v_{\left\lceil\frac{x}{2}\right\rceil-2} P^{-1} v_{1} v_{s} & \text { if } 1 \in\left\{y_{1}, \ldots, y_{t_{2}}\right\} \text { and } u_{x^{\prime}} u_{l+1} \text { has color } i_{l+\left\lceil\frac{x}{2}\right\rceil-2} \\ v_{1} P v_{\left\lceil\frac{x}{2}\right\rceil-2} v_{s} & \text { if }\left\lceil\frac{x}{2}\right\rceil-2 \in\left\{y_{1}, \ldots, y_{t_{2}}\right\} \text { and } u_{x^{\prime}} u_{l+1} \text { has color } i_{l+\left\lceil\frac{x}{2}\right\rceil-2} .\end{cases}
$$




$$
P^{\prime}= \begin{cases}P_{1} u_{x} P u_{l+1} u_{x^{\prime}} P^{-1} u_{x^{\prime}-\left\lfloor\frac{x}{2}\right\rfloor} & \text { if }\left\lfloor\frac{x}{2}\right\rfloor+2 \leq x^{\prime} \leq x-1 \\ P_{1} u_{x} P u_{l+1} u_{x^{\prime}} P u_{x^{\prime}+\left\lfloor\frac{x}{2}\right\rfloor} & \text { if } 2 \leq x^{\prime} \leq\left\lfloor\frac{x}{2}\right\rfloor .\end{cases}
$$

Note that if $2 \leq x^{\prime} \leq\left\lfloor\frac{x}{2}\right\rfloor$, then $x^{\prime}+\left\lfloor\frac{x}{2}\right\rfloor \leq 2\left\lfloor\frac{x}{2}\right\rfloor=x-1$, and so $P^{\prime}$ is a heterochromatic path of length $l+1$, a contradiction to the choice of $P$.

So, we get that $i_{1} \notin C\left(\left\{u_{1} v_{s}, u_{2} v_{s}, \ldots, u_{l} v_{s}\right\}\right)$.

Now we turn to proving our main theorem.

\section{Proof of Theorem 3.1}

Proof. We will use induction on $k$ to prove the theorem.

For $k=8$, by Theorem 2.1 there is a heterochromatic path of length $6=\left\lceil\frac{3 k}{5}\right\rceil+1$ in $G$.

Suppose now $k \geq 9$, and the theorem is true for all graphs $G$ such that $d^{c}(v) \geq k^{\prime}$ for every vertex $v$ in $G$ with $8 \leq k^{\prime} \leq k-1$. In the following, all the notations are the same as in Section 3. Since $d^{c}(v) \geq k>k-1$ for every vertex $v$ of $G, G$ has a heterochromatic path of length $\left\lceil\frac{3(k-1)}{5}\right\rceil+1$. It remains to show that $l \geq\left\lceil\frac{3 k}{5}\right\rceil+1$, which implies that $P^{\prime}=u_{1} P u_{\left\lceil\frac{3 k}{5}\right\rceil+2}$ is a heterochromatic path of length $\left\lceil\frac{3 k}{5}\right\rceil+1$. We will proceed by contradictions. Suppose that $l \leq\left\lceil\frac{3 k}{5}\right\rceil$. On the other hand, by induction hypothesis we have that $l \geq\left\lceil\frac{3(k-1)}{5}\right\rceil+1 \geq\left\lceil\frac{3 k}{5}\right\rceil$. Then $l=\left\lceil\frac{3 k}{5}\right\rceil$ and $k \equiv 1,2,4(\bmod 5)$. We will distinguish three cases: $t_{1} \geq 2, t_{1}=1$ and $t_{1}=0$.

Case $1 t_{1} \geq 2$.

By Lemma 3.9, there is no $x_{j}\left(1 \leq j \leq t_{1}\right)$ such that $C\left(\left\{u_{1} u_{l+1}, u_{2} u_{l+1} \ldots, u_{x_{j}-1} u_{l+1}\right\}\right)-$ $\left\{i_{1}, i_{2}, \ldots, i_{l}, i_{l+1}, \ldots, i_{l+s-1}, i_{l+s+j-1}\right\} \neq \emptyset$. So, $C\left(\left\{u_{1} u_{l+1}, \ldots, u_{x_{t_{1}}-1} u_{l+1}\right\}\right) \subseteq\left\{i_{1}, i_{2}, \ldots\right.$, $\left.i_{l+s-1}, i_{l+s+t_{1}-1}\right\}$. Then, by Lemma 3.10 there is no $x_{1} \leq x^{\prime} \leq x_{t_{1}}-1$ such that $C\left(u_{x^{\prime}} u_{l+1}\right) \in$ $\left\{i_{l+1}, \ldots, i_{l+s-x_{t_{1}}+x_{1}+2 t_{1}-4}\right\} \cup\left\{i_{l+\left[\frac{s-t_{2}-1}{2}\right\rceil+x_{t_{1}}-x_{1}-2 t_{1}+2}, \ldots i_{l+s-1}\right\}$. Now we will distinguish the following two cases:

Case $1.1 s-x_{t_{1}}+x_{1}+2 t_{1}-4 \leq\left\lceil\frac{s-t_{2}-1}{2}\right\rceil+x_{t_{1}}-x_{1}-2 t_{1}+1$. Since $\mid\left\{s-x_{t_{1}}+\right.$ $\left.x_{1}+2 t_{1}-3, \ldots,\left\lceil\frac{s-t_{2}-1}{2}\right\rceil+x_{t_{1}}-x_{1}-2 t_{1}+1\right\} \mid=\left\lceil\frac{s-t_{2}-1}{2}\right\rceil-s+2\left(x_{t_{1}}-x_{1}-2 t_{1}\right)+$ 5 , there are at least $(l-1)-\left[\left(k_{0}-1\right)+\left(\left\lceil\frac{s-t_{2}-1}{2}\right\rceil-s+2\left(x_{t_{1}}-x_{1}-2 t_{1}\right)+5\right)+\right.$ $\left.\left((l-1)-\left(k-l+k_{0}-1\right)\right)\right]=k-l-\left\lceil\frac{s-t_{2}-1}{2}\right\rceil+s-2\left(x_{t_{1}}-x_{1}-2 t_{1}\right)-5$ different colors not in $\left\{i_{1}, i_{2}, \ldots, i_{l}, i_{l+\left(s-x_{t_{1}}+x_{1}+2 t_{1}-3\right)}, \ldots, i_{l+\left(\left\lceil\frac{s-t_{2}-1}{2}\right\rceil+x_{t_{1}}-x_{1}-2 t_{1}+1\right)}\right\}$ that belong to $C\left(\left\{u_{1} u_{l+1}, u_{2} u_{l+1}, \ldots, u_{l-1} u_{l+1}\right\}\right)$. So, there are at least

$$
\begin{aligned}
& k-l-\left\lceil\frac{s-t_{2}-1}{2}\right\rceil+s-2\left(x_{t_{1}}-x_{1}-2 t_{1}\right)-5-\left(l-1-x_{t_{1}}+1\right)-1 \\
= & k-2 l+s-\left\lceil\frac{s-t_{2}-1}{2}\right\rceil-x_{t_{1}}+2 x_{1}+4 t_{1}-6 \\
\geq & k-2 l+s-\left\lceil\frac{s-\left(k-l-s+1-t_{1}\right)-1}{2}\right\rceil-(l-s+1)+2 s+2+4 t_{1}-6 \\
\geq & \left(3 k-7 l+6 s+7 t_{1}-9\right) / 2 \\
\geq & (3 k-7 l+6 s+7 \max \{k-l-2 s+3,2\}-9) / 2
\end{aligned}
$$




$$
\begin{aligned}
& = \begin{cases}(10 k-14 l-8 s+12) / 2 & \text { if } s \leq 2 l-k-1 \text { and } k \equiv 1,4(\bmod 5) \\
(10 k-14 l-8 s+12) / 2 & \text { if } s \leq 2 l-k-2 \text { and } k \equiv 2(\bmod 5) \\
(3 k-7 l+6 s+5) / 2 & \text { if } s=2 l-k \text { and } k \equiv 1,4(\bmod 5) \\
(3 k-7 l+6 s+5) / 2 & \text { if } s=2 l-k, 2 l-k-1 \text { and } k \equiv 2(\bmod 5)\end{cases} \\
& \geq \begin{cases}(18 k-30 l+20) / 2 & \text { if } s \leq 2 l-k-1 \text { and } k \equiv 1,4(\bmod 5) \\
(18 k-30 l+28) / 2 & \text { if } s \leq 2 l-k-2 \text { and } k \equiv 2(\bmod 5) \\
(5 l-3 k+5) / 2 & \text { if } s=2 l-k \text { and } k \equiv 1,4(\bmod 5) \\
(5 l-3 k-1) / 2 & \text { if } s=2 l-k, 2 l-k-1 \text { and } k \equiv 2(\bmod 5)\end{cases}
\end{aligned}
$$

colors in $\left\{i_{l+1}, i_{l+2}, \ldots, i_{l+s-x_{t_{1}}+x_{1}+2 t_{1}-4}, i_{l+\left\lceil\frac{s-t_{2}-1}{2}\right\rceil+x_{t_{1}}-x_{1}-2 t_{1}+2}, \ldots, i_{l+s-1}\right\}$ that belong to $C\left(\left\{u_{1} u_{l+1}, u_{2} u_{l+1}, \ldots, u_{x_{t_{1}}-1} u_{l+1}\right\}\right)$, i.e., they belong to $C\left(\left\{u_{1} u_{l+1}, u_{2} u_{l+1}, \ldots, u_{x_{1}-1} u_{l+1}\right\}\right)$. Note that

$$
\begin{aligned}
& \left|\left\{1,2, \ldots, s-\left\lceil\frac{x_{1}-1}{2}\right\rceil\right\} \cup\left\{\left\lceil\frac{s+x_{1}-t_{2}}{2}\right\rceil-2,\left\lceil\frac{s+x_{1}-t_{2}}{2}\right\rceil-1, \ldots, s-1\right\}\right| \\
= & s-\left\lceil\frac{x_{1}-1}{2}\right\rceil+s-\left\lceil\frac{s+x_{1}-t_{2}}{2}\right\rceil+2=2 s-\left\lceil\frac{x_{1}-1}{2}\right\rceil-\left\lceil\frac{s+x_{1}-t_{2}}{2}\right\rceil+2, \\
& \mid\left(\{ 1 , 2 , \ldots , s - x _ { t _ { 1 } } + x _ { 1 } + 2 t _ { 1 } - 4 \} \cup \left\{\left\lceil\frac{s-t_{2}-1}{2}\right\rceil+x_{t_{1}}-x_{1}-2 t_{1}+2,\right.\right. \\
& \quad \cdots, s-1\} \mid \\
= & s-x_{t_{1}}+x_{1}+2 t_{1}-4+s-\left\lceil\frac{s-t_{2}-1}{2}\right\rceil-x_{t_{1}}+x_{1}+2 t_{1}-2 \\
= & 2 s-2 x_{t_{1}}+2 x_{1}+4 t_{1}-\left\lceil\frac{s-t_{2}-1}{2}\right\rceil-6, \\
& \left(2 s-2 x_{t_{1}}+2 x_{1}+4 t_{1}-\left\lceil\frac{s-t_{2}-1}{2}\right\rceil-6\right)-\left(2 s-\left\lceil\frac{x_{1}-1}{2}\right\rceil-\left\lceil\frac{s+x_{1}-t_{2}}{2}\right\rceil+2\right) \\
= & \left(k-2 l+s-\left\lceil\frac{s-t_{2}-1}{2}\right\rceil-x_{t_{1}}+2 x_{1}+4 t_{1}-6\right) \\
+ & \left(\left\lceil\frac{x_{1}-1}{2}\right\rceil+\left\lceil\frac{s+x_{1}-t_{2}}{2}\right\rceil-x_{t_{1}}-k+2 l-s-2\right) \\
&
\end{aligned}
$$

and

$$
\begin{aligned}
& \left\lceil\frac{x_{1}-1}{2}\right\rceil+\left\lceil\frac{s+x_{1}-t_{2}}{2}\right\rceil-x_{t_{1}}-k+2 l-s-2 \\
\leq & \frac{x_{1}+s+x_{1}-\left(k-l-s+1-t_{1}\right)+1}{2}-x_{t_{1}}-k+2 l-s-2 \\
= & x_{1}-x_{t_{1}}-k+2 l-2+\frac{l+t_{1}-k}{2}=x_{1}+2\left(t_{1}-1\right)-x_{t_{1}}-\frac{3 k-5 l+3 t_{1}}{2} \\
\leq & -\frac{6+3 k-5 l}{2} \leq-1 .
\end{aligned}
$$

So, $\left.2 s-2 x_{t_{1}}+2 x_{1}+4 t_{1}-\left\lceil\frac{s-t_{2}-1}{2}\right\rceil-6\right)-\left(2 s-\left\lceil\frac{x_{1}-1}{2}\right\rceil-\left\lceil\frac{s+x_{1}-t_{2}}{2}\right\rceil+2\right)<k-2 l+$ $s-\left\lceil\frac{s-t_{2}-1}{2}\right\rceil-x_{t_{1}}+2 x_{1}+4 t_{1}-6, C\left(\left\{u_{1} u_{l+1}, \ldots, u_{x_{1}-1} u_{l+1}\right\}\right) \cap\left\{i_{l+1}, \ldots, i_{l+s-\left\lceil\frac{x_{1}-1}{2}\right\rceil}\right.$, $\left.i_{l+\left\lceil\frac{s+x_{1}-t_{2}}{2}\right\rceil-2}, \ldots, i_{l+s-1}\right\} \neq \emptyset$. By Lemma 3.6, there is a heterochromatic path of length $l+1$, a contradiction to the choice of $P$.

Case $1.2 s-x_{t_{1}}+x_{1}+2 t_{1}-4 \geq\left\lceil\frac{s-t_{2}-1}{2}\right\rceil+x_{t_{1}}-x_{1}-2 t_{1}+2$. Then there are at least $(l-1)-\left[\left(k_{0}-1\right)+(l-1)-\left(k-l+k_{0}-1\right)\right]=k-l$ colors not in $\left\{i_{1}, i_{2}, \ldots, i_{l}\right\}$ that belong to $C\left(\left\{u_{1} u_{l+1}, \ldots, u_{l-1} u_{l+1}\right\}\right)$. So, there are at least

$$
\begin{aligned}
& k-l-\left(l-1-x_{t_{1}}+1\right)-1 \\
= & k-2 l+x_{t_{1}}-1 \\
\geq & k-2 l+s+1+2 t_{1}-3 \\
\geq & k-2 l+s+2 \max \{k-l-2 s+3,2\}-2
\end{aligned}
$$




$$
\begin{aligned}
& = \begin{cases}3 k-4 l-3 s+4 & \text { if } s \leq 2 l-k-1 \text { and } k \equiv 1,4(\bmod 5) \\
3 k-4 l-3 s+4 & \text { if } s \leq 2 l-k-2 \text { and } k \equiv 2(\bmod 5) \\
k-2 l+s+2 & \text { if } s=2 l-k \text { and } k \equiv 1,4(\bmod 5) \\
k-2 l+s+2 & \text { if } s=2 l-k, 2 l-k-1 \text { and } k \equiv 2(\bmod 5)\end{cases} \\
& \geq \begin{cases}6 k-10 l+7 & \text { if } s \leq 2 l-k-1 \text { and } k \equiv 1,4(\bmod 5) \\
6 k-10 l+10 & \text { if } s \leq 2 l-k-2 \text { and } k \equiv 2(\bmod 5) ; \\
2 & \text { if } s=2 l-k \text { and } k \equiv 1,4(\bmod 5) ; \\
1 & \text { if } s=2 l-k, 2 l-k-1 \text { and } k \equiv 2(\bmod 5)\end{cases}
\end{aligned}
$$

colors in $\left\{i_{l+1}, i_{l+2}, \ldots, i_{l+s-1}\right\}$ that belong to $C\left(\left\{u_{1} u_{l+1}, u_{2} u_{l+1}, \ldots, u_{x_{t_{1}}-1} u_{l+1}\right\}\right)$, i.e., they belong to $C\left(\left\{u_{1} u_{l+1}, u_{2} u_{l+1}, \ldots, u_{x_{1}-1} u_{l+1}\right\}\right)$.

Since

$$
\begin{aligned}
& (s-1)-\left(2 s-\left\lceil\frac{x_{1}-1}{2}\right\rceil-\left\lceil\frac{s+x_{1}-t_{2}}{2}\right\rceil+2\right) \\
= & \left\lceil\frac{x_{1}-1}{2}\right\rceil+\left\lceil\frac{s+x_{1}-t_{2}}{2}\right\rceil-s-3 \\
= & \left(k-2 l+x_{t_{1}}-1\right)+\left(\left\lceil\frac{x_{1}-1}{2}\right\rceil+\left\lceil\frac{s+x_{1}-t_{2}}{2}\right\rceil-s-k+2 l-x_{t_{1}}-2\right) \\
< & k-2 l+x_{t_{1}}-1
\end{aligned}
$$

we have that $C\left(\left\{u_{1} u_{l+1}, u_{2} u_{l+1}, \ldots, u_{x_{1}-1} u_{l+1}\right\}\right) \cap\left\{i_{l+1}, \ldots, i_{l+s-\left\lceil\frac{x_{1}-1}{2}\right\rceil}, i_{l+\left\lceil\frac{s+x_{1}-t_{2}}{2}\right\rceil-1}, \ldots\right.$, $\left.i_{l+s-1}\right\} \neq \emptyset$. By Lemma 3.6, there is a heterochromatic path of length $l+1$, a contradiction to the choice of $P$.

Case $2 t_{1}=1$. Then, by Lemma 3.4 we have $1=t_{1} \geq k-l-2 s+3$, and so $2 s \geq k-l+2$. On the other hand, $s \leq 2 l-k$. Since $2(2 l-k)-(k-l+2)=5 l-3 k-2$, we have that $s=k_{0}=2 l-k$ if $k \equiv 1,4(\bmod 5)$ or $2 l-k-1 \leq s \leq k_{0} \leq 2 l-k$ if $k \equiv 2(\bmod 5)$.

By Lemma 3.9, we have that $C\left(\left\{u_{1} u_{l+1}, \ldots, u_{x_{1}-1} u_{l+1}\right\}\right) \subseteq\left\{i_{1}, i_{2}, \ldots, i_{l+s-1}, i_{l+s}\right\}$. On the other hand, if there exists an $x_{1}-s \leq x \leq s$ such that $u_{x} u_{l+1}$ has a color in $\left\{i_{1}, i_{2}, \ldots, i_{k_{0}-1}\right\}$, let $P_{1}^{\prime}=u_{x} P^{-1} u_{x-\left(x_{1}-s\right)+1}$ and $P_{1}^{\prime \prime}=u_{x} P u_{x+\left(x_{1}-s\right)-1}$, then there is a $P_{1} \in\left\{P_{1}^{\prime}, P_{1}^{\prime \prime}\right\}$ such that $C\left(u_{x} u_{l+1}\right) \cap C\left(P_{1}\right)=\emptyset$, and so $P^{\prime}=v_{1} P v_{s} u_{x_{1}} P u_{l+1} P_{1}$ is a heterochromatic path of length $l+1$, a contradiction to the choice of $P$. So, $C\left(\left\{u_{x_{1}-s} u_{l+1}, \ldots, u_{s} u_{l+1}\right\}\right) \cap\left\{i_{1}, i_{2}, \ldots, i_{k_{0}-1}\right\}=\emptyset$. We consider the following two cases:

Case $2.1 s-\left(x_{1}-s\right)+1 \geq\left(x_{1}-1\right)-\left(k_{0}-1\right)+1 \geq 0$, i.e., $2 x_{1} \leq 2 s+k_{0}$. Then, there are at least $\left[s-\left(x_{1}-s\right)+1\right]-\left[(l-1)-\left(k-l+k_{0}-1\right)\right]-1>0($ $\left[s-\left(x_{1}-s\right)+1\right]-\left[(l-1)-\left(k-l+k_{0}-1\right)\right]-1=2 s-x_{1}+1-l+1+k-l+k_{0}-1-1=$ $k-2 l+2 s+k_{0}-x_{1} \geq k-2 l+2 x_{1}-x_{1} \geq k-2 l+s+1 \geq k-2 l+\frac{k-l+2}{2}+1=\frac{3 k-5 l+4}{2} \geq 0$, and so $\left[s-\left(x_{1}-s\right)+1\right]-\left[(l-1)-\left(k-l+k_{0}-1\right)\right]-1=0$ if and only if $2(s+1)=2 x_{1}=$ $2 s+k_{0} \geq 3 s$, i.e., $s \leq 2$. On the other hand, $k \geq 8$ implies that $s \geq 3$, and so we have $\left[s-\left(x_{1}-s\right)+1\right]-\left[(l-1)-\left(k-l+k_{0}-1\right)\right]-1>0$. $)$ different colors in $\left\{i_{l+1}, \ldots, i_{l+s-1}\right\}$ that belong to $C\left(\left\{u_{x_{1}-s} u_{l+1}, \ldots, u_{s} u_{l+1}\right\}\right) \subseteq C\left(\left\{u_{1} u_{l+1}, \ldots, u_{x_{1}-1} u_{l+1}\right\}\right)$. Since $\mid\left\{s-\left\lceil\frac{x_{1}-1}{2}\right\rceil+\right.$ $\left.1, \ldots,\left\lceil\frac{s+x_{1}-t_{2}}{2}\right\rceil-3\right\} \mid \leq\left\lceil\frac{s+x_{1}-\left(k-l-s+1-t_{1}\right)}{2}\right\rceil+\left\lceil\frac{x_{1}-1}{2}\right\rceil-s-3=\left\lceil\frac{2 s-k+l+x_{1}}{2}\right\rceil+\left\lceil\frac{x_{1}-1}{2}\right\rceil-s-3=$ $s+\left\lceil\frac{l-k+x_{1}}{2}\right\rceil+\left\lceil\frac{x_{1}-1}{2}\right\rceil-s-3 \leq\left\lceil\frac{l-k}{2}\right\rceil+x_{1}-3=\left(k-2 l+2 s+k_{0}-x_{1}\right)+\left(\left\lceil\frac{l-k}{2}\right\rceil+2 x_{1}-\right.$ $\left.2 s+2 l-k-k_{0}-3\right) \leq\left(k-2 l+2 s+k_{0}-x_{1}\right)+\left(\left\lceil\frac{l-k}{2}\right\rceil+k_{0}-k+2 l-k_{0}-3\right) \leq(k-2 l+2 s+$ $\left.k_{0}-x_{1}\right)+\left(\frac{5 l-3 k+1}{2}-3\right)<k-2 l+2 s+k_{0}-x_{1}$, we have that $C\left(\left\{u_{1} u_{l+1}, \ldots, u_{x_{1}-1} u_{l+1}\right\}\right) \cap$ $\left\{i_{l+1}, i_{l+2}, \ldots, i_{l+s-\left\lceil\frac{x_{1}-1}{2}\right\rceil}, i_{l+\left\lceil\frac{s+x_{1}-t_{2}}{2}\right\rceil-2}, i_{l+\left\lceil\frac{s+x_{1}-t_{2}}{2}\right\rceil-1}, \ldots, i_{l+s-1}\right\} \neq \emptyset$. Then, by Lemma 
3.6 there is a heterochromatic path of length $l+1$, a contradiction to the choice of $P$.

Case $2.20<s-\left(x_{1}-s\right)+1 \leq\left(x_{1}-1\right)-\left(k_{0}-1\right)$, i.e., $2 x_{1} \geq 2 s+k_{0}+1$. Then there are at least $\left[\left(x_{1}-1\right)-\left(k_{0}-1\right)\right]-\left[(l-1)-\left(k-l+k_{0}-1\right)\right]-1=$ $x_{1}-2 l+k-1 \geq s+\frac{k_{0}+1}{2}-2 l+k-1 \geq \frac{3 s-1}{2}-2 l+k \geq \frac{3 k-3 l+4}{4}-2 l+k=\frac{7 k-11 l+4}{4}$ $\left(=0\right.$ if $k=12, l=8, s=k_{0}=3, x_{1}=5$ and $\geq 1$ otherwise) different colors in $\left\{i_{l+1}, i_{l+2}, \ldots, i_{l+s-1}\right\}$ that belong to $C\left(\left\{u_{1} u_{l+1}, u_{2} u_{l+1}, \ldots, u_{x_{1}-1} u_{l+1}\right\}\right)$. We first consider the case when $k=12, l=8, s=k_{0}=3$ and $x_{1}=5$. There are at least $x_{1}-2-[(l-1)-$ $\left.\left(k-l+k_{0}-1\right)\right]-1=5-2-(7-(12-8+3-1))-1=1$ colors in $\left\{i_{1}, i_{2}, i_{9}, i_{10}\right\}$ that belong to $C\left(u_{2} u_{9}, u_{3} u_{9}, u_{4} u_{9}\right)$, i.e., there is an $2 \leq x \leq 4$ such that $C\left(u_{x} u_{9}\right) \in\left\{i_{1}, i_{2}, i_{9}, i_{10}\right\}$. If $C\left(u_{x} u_{9}\right) \in\left\{i_{1}, i_{2}\right\}$, then $v_{1} v_{2} v_{3} u_{5} P u_{9} u_{x} u_{x-1}$ or $v_{1} v_{2} v_{3} u_{5} P u_{9} u_{x} u_{x+1}$ is a heterochromatic path of length 9 ; if $C\left(u_{x} u_{9}\right)=i_{10}$, then $v_{2} v_{1} v_{3} u_{5} P u_{9} u_{x} u_{x-1}$ is a heterochromatic path of length 9 ; if $C\left(u_{x} u_{9}\right)=i_{9}$, then $v_{2} u_{5} P u_{9} u_{x} u_{x-1} u_{x-2}$ or $v_{2} u_{5} P u_{9} u_{x} u_{x+1} u_{x+2}$ is a heterochromatic path of length 9 , a contradiction to the choice of $P$.

Next we shall only consider the case when $x_{1}-2 l+k-1 \geq 1$. If there exists an $1 \leq x \leq x_{1}-1$ such that $u_{x} u_{l+1}$ has a color in $\left\{i_{l+1}, i_{l+2}, \ldots, i_{l+s-\left\lceil\frac{x_{1}-1}{2}\right\rceil}, i_{l+\left\lceil\frac{s+x_{1}-t_{2}}{2}\right\rceil-2}\right.$, $\left.i_{l+\left\lceil\frac{s+x_{1}-t_{2}}{2}\right\rceil-1}, \ldots, i_{l+s-1}\right\}$, then by Lemma 3.6 there is a heterochromatic path of length $l+$ 1 in $G$, a contradiction to the choice of $P$. Otherwise, since $\mid\left\{s-\left\lceil\frac{x_{1}-1}{2}\right\rceil+1, \ldots,\left\lceil\frac{s+x_{1}-t_{2}}{2}\right\rceil-\right.$ $3\} \mid \leq\left\lceil\frac{s+x_{1}-\left(k-l-s+1-t_{1}\right)}{2}\right\rceil+\left\lceil\frac{x_{1}-1}{2}\right\rceil-s-3=\left\lceil\frac{2 s-k+l+x_{1}}{2}\right\rceil+\left\lceil\frac{x_{1}-1}{2}\right\rceil-s-3=s+\left\lceil\frac{l-k+x_{1}}{2}\right\rceil+$ $\left\lceil\frac{x_{1}-1}{2}\right\rceil-s-3 \leq\left\lceil\frac{l-k}{2}\right\rceil+x_{1}-3=\left(x_{1}-2 l+k-1\right)+\left(\left\lceil\frac{l-k}{2}\right\rceil+2 l-k-2\right)=\left(x_{1}-2 l+k-1\right)+$ $\left(\left\lceil\frac{5 l-3 k}{2}\right\rceil-2\right) \leq x_{1}-2 l+k-1$, we have that $\left\{i_{1}, i_{2}, \ldots, i_{k_{0}-1}, i_{l+s-\left\lceil\frac{x_{1}-1}{2}\right\rceil+1}, \ldots, i_{l+\left\lceil\frac{s+x_{1}-t_{2}}{2}\right\rceil-3}\right\}$ $\subseteq C\left(\left\{u_{1} u_{l+1}, \ldots, u_{x_{1}-1} u_{l+1}\right\}\right)$, and $k \equiv 2,4(\bmod 5)$ as well as $t_{2}=k-l-s$ if $k \equiv$ $4(\bmod 5), t_{2}=k-l-s$ or $k-l-s+1$ if $k \equiv 2(\bmod 5)$. We distinguish two subcases:

Case 2.2.1 If $k \equiv 2(\bmod 5)$, then $4 l-2 k-(k-l+2)=5 l-3 k-2=2$, and so $s=2 l-k-1$ or $2 l-k$.

If $s=2 l-k-1$, then $s-2 \geq t_{2} \geq k-l-s+1-1=s+(k-l-2 s)=$ $s+(k-l-4 l+2 k+2)=s-2, x_{1}-2 l+k-1=x_{1}-s-2$ and $\left|\left\{\left\lfloor\frac{s}{2}\right\rfloor+3, \ldots, x_{1}-\left\lceil\frac{s}{2}\right\rceil-2\right\}\right|=$ $x_{1}-\left\lceil\frac{s}{2}\right\rceil-\left\lfloor\frac{s}{2}\right\rfloor-4=x_{1}-s-4$.

Since $k \geq 8$ and $s \geq 3$, we have that $t_{2}=s-2>0$. If $s \equiv 0(\bmod 2)$, then there exists some $x \in\left\{1,2, \ldots, \frac{s}{2}+1\right\} \cup\left\{x_{1}-\frac{s}{2}-1, \ldots, x_{1}-1\right\}$ such that $u_{x} u_{l+1}$ has a color in $\left\{i_{l+s-\left\lceil\frac{x_{1}-1}{2}\right\rceil+1}, \ldots, i_{l+\left\lceil\frac{s+x_{1}-t_{2}}{2}\right\rceil-3}\right\}$. Let

$$
\begin{aligned}
& P_{1}= \begin{cases}v_{\frac{s}{2}} P^{-1} v_{1} v_{s} & \text { if } u_{x} u_{l+1} \text { has a color in }\left\{i_{l+\frac{s}{2}}, \ldots, i_{l+\left\lceil\frac{s+x_{1}-t_{2}}{2}\right\rceil-3}\right\} ; \\
v_{\frac{s}{2}} P v_{s} & \text { if } u_{x} u_{l+1} \text { has a color in }\left\{i_{l+s-\left\lceil\frac{x_{1}-1}{2}\right\rceil+1}, \ldots, i_{l+\frac{s}{2}-1}\right\} .\end{cases} \\
& P_{2}= \begin{cases}u_{x} P^{-1} u_{x-\left(x_{1}-\frac{s}{2}-2\right)} & \text { if } x_{1}-\frac{s}{2}-1 \leq x \leq x_{1}-1 ; \\
u_{x} P u_{x+\left(x_{1}-\frac{s}{2}-2\right)} & \text { if } 1 \leq x \leq \frac{s}{2}+1 .\end{cases}
\end{aligned}
$$

Then, $P^{\prime}=P_{1} u_{x_{1}} P u_{l+1} P_{2}$ is a heterochromatic path of length $l+1$, a contradiction to the choice of $P$.

If $s \equiv 1(\bmod 2)$, then there exists some $x \in\left\{1,2, \ldots,\left\lfloor\frac{s}{2}\right\rfloor+2\right\} \cup\left\{x_{1}-\left\lceil\frac{s}{2}\right\rceil-1, \ldots, x_{1}-\right.$ $\left.2, x_{1}-1\right\}$ such that $u_{x} u_{l+1}$ has a color in $\left\{i_{l+s-\left\lceil\frac{x_{1}-1}{2}\right\rceil+1}, \ldots, i_{l+\left\lceil\frac{s}{2}\right\rceil-2}\right.$, 


$$
\begin{aligned}
i_{l+\left\lceil\frac{s}{2}\right\rceil}, \ldots, & \left.i_{l+\left\lceil\frac{s+x_{1}-t_{2}}{2}\right\rceil-3}\right\} . \text { Let } \\
P_{1} & = \begin{cases}v_{\left\lceil\frac{s}{2}\right\rceil} P^{-1} v_{1} v_{s} & \text { if } u_{x} u_{l+1} \text { has a color in }\left\{i_{l+\left\lceil\frac{s}{2}\right\rceil}, \ldots, i_{l+\left\lceil\frac{s+x_{1}-t_{2}}{2}\right\rceil-3}\right\} ; \\
v_{\left\lceil\frac{s}{2}\right\rceil-1} P v_{s} & \text { if } u_{x} u_{l+1} \text { has a color in }\left\{i_{l+s-\left\lceil\frac{x_{1}-1}{2}\right\rceil+1}, \ldots, i_{l+\left\lceil\frac{s}{2}\right\rceil-2}\right\} .\end{cases} \\
P_{2} & = \begin{cases}u_{x} P^{-1} u_{x-\left(x_{1}-\left\lceil\frac{s}{2}\right\rceil-2\right)} & \text { if } x_{1}-\left\lceil\frac{s}{2}\right\rceil-1 \leq x \leq x_{1}-1 ; \\
u_{x} P u_{x+\left(x_{1}-\left\lceil\frac{s}{2}\right\rceil-2\right)} & \text { if } 1 \leq x \leq\left\lfloor\frac{s}{2}\right\rfloor+2 .\end{cases}
\end{aligned}
$$

Then, $P^{\prime}=P_{1} u_{x_{1}} P u_{l+1} P_{2}$ is a heterochromatic path of length $l+1$, a contradiction to the choice of $P$.

If $s=2 l-k$, then $x_{1}-2 l+k-1=x_{1}-s-1$ and $t_{2} \geq k-l-s=s-4 \geq 0$.

We first consider the case when $t_{2}=0$. Then $k=12, l=8, k_{0}=s=4$, and $5=s+1 \leq$ $x_{1} \leq l-s+1=5$ which implies $x_{1}=5$. If there exists a $v \notin\left\{u_{5}, u_{6}, u_{7}\right\}$ such that $u_{9} v$ has a color not in $\left\{i_{4}, \ldots, i_{12}\right\}$, then $v \notin\left\{u_{5}, \ldots, u_{8}, v_{1}, \ldots, v_{4}\right\}$ and $P^{\prime}=v_{1} P v_{4} u_{5} P u_{9} v$ is a heterochromatic path of length $l+1$, a contradiction. So, $i_{9} \in C\left(\left\{u_{1} u_{9}, u_{2} u_{9}, u_{3} u_{9}, u_{4} u_{9}\right\}\right)$. Let

$$
P^{\prime}= \begin{cases}v_{2} P v_{4} u_{5} P u_{9} u_{1} u_{2} & \text { if } u_{1} u_{9} \text { has color } i_{9} \\ v_{2} P v_{4} u_{5} P u_{9} u_{x} u_{x-1} & \text { if } u_{x} u_{9}(2 \leq x \leq 4) \text { has color } i_{9} .\end{cases}
$$

Then, $P^{\prime}$ is a heterochromatic path of length $l+1$, a contradiction.

Next we consider the case when $t_{2}>0$. Since if $s \geq 5$, we have that $2<\left\lceil\frac{s}{2}\right\rceil \leq$ $\frac{s+1}{2} \leq s-2$, and so $\left\{1,2,\left\lceil\frac{s}{2}\right\rceil\right\} \cap\left\{y_{1}, y_{2}, \ldots, y_{t_{2}}\right\} \neq \emptyset$. If $s \equiv 0(\bmod 2)$. Then there exists some $x \in\left\{1,2, \ldots, \frac{s}{2}+1\right\} \cup\left\{x_{1}-\frac{s}{2}-1, \ldots, x_{1}-1\right\}$ such that $u_{x} u_{l+1}$ has a color in $\left\{i_{l+s-\left\lceil\frac{x_{1}-1}{2}\right\rceil+1}, \ldots, i_{l+\frac{s}{2}-1}, i_{l+\frac{s}{2}+1}, \ldots, i_{l+\left\lceil\frac{s+x_{1}-t_{2}}{2}\right\rceil-2}\right\}$. Let

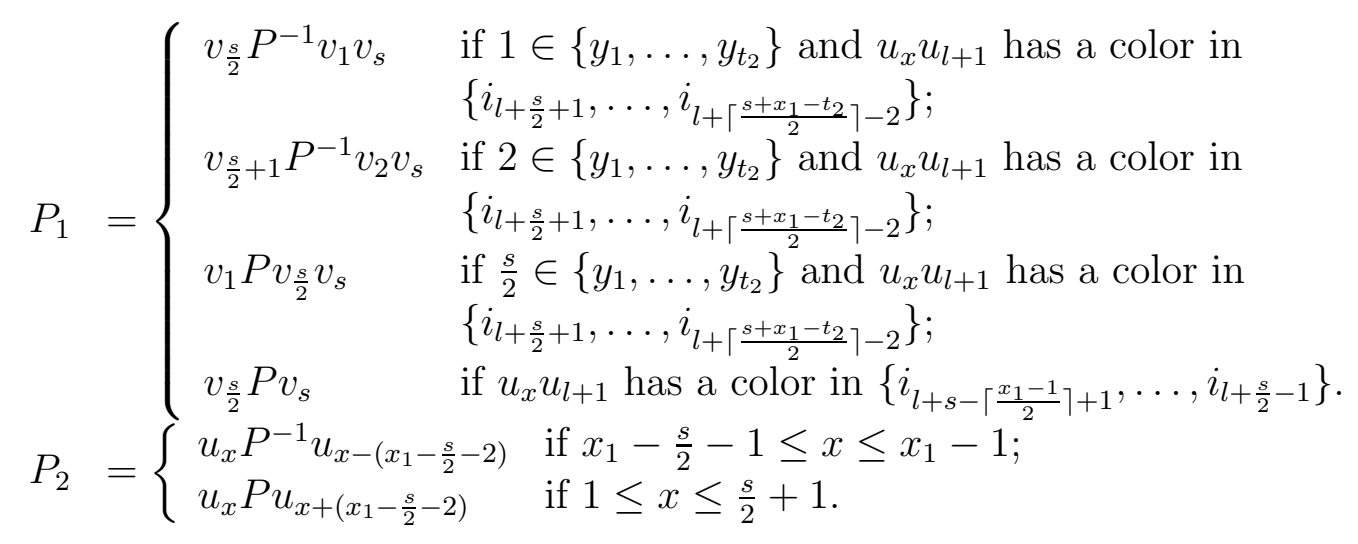

Then, $P^{\prime}=P_{1} u_{x_{1}} P u_{l+1} P_{2}$ is a heterochromatic path of length $l+1$, a contradiction to the choice of $P$.

If $s \equiv 1(\bmod 2)$, then there exists some $x \in\left\{1,2, \ldots, \frac{s+3}{2}\right\} \cup\left\{x_{1}-\frac{s+3}{2}, \ldots, x_{1}-1\right\}$ such that $u_{x} u_{l+1}$ has a color in $\left\{i_{l+s-\left\lceil\frac{x_{1}-1}{2}\right\rceil+1}, \ldots, i_{l+\frac{s-3}{2}}, i_{l+\frac{s+3}{2}}, \ldots, i_{l+\left\lceil\frac{s+x_{1}-t_{2}}{2}\right\rceil-2}\right\}$. Let 


$$
\begin{aligned}
& P_{1}= \begin{cases}v_{\frac{s+1}{2}} P^{-1} v_{1} v_{s} & \text { if } 1 \in\left\{y_{1}, \ldots, y_{t_{2}}\right\} \text { and } u_{x} u_{l+1} \text { has a color in } \\
& \left\{i_{l+\frac{s+3}{2}}, \ldots, i_{l+\left\lceil\frac{s+x_{1}-t_{2}}{2}\right\rceil-2}\right\} ; \\
v_{\frac{s+3}{2}} P^{-1} v_{2} v_{s} \quad & \text { if } 2 \in\left\{y_{1}, \ldots, y_{t_{2}}\right\}^{2} \text { and } u_{x} u_{l+1} \text { has a color in } \\
& \left\{i_{l+\frac{s+3}{2}}, \ldots, i_{l+\left\lceil\frac{s+x_{1}-t_{2}}{2}\right\rceil-2}\right\} ; \\
v_{1} P v_{\frac{s+1}{2}} v_{s} \quad & \text { if } \frac{s+1}{2} \in\left\{y_{1}, \ldots, y_{t_{2}}\right\} \text { and } u_{x} u_{l+1} \text { has a color in } \\
& \left\{i_{l+\frac{s+3}{2}}, \ldots, i_{l+\left\lceil\frac{s+x_{1}-t_{2}}{2}\right\rceil-2}\right\} ; \\
v_{\frac{s-1}{2}} P v_{s} & \text { if } u_{x} u_{l+1} \text { has a color in }\left\{i_{l+s-\left\lceil\frac{x_{1}-1}{2}\right\rceil+1}, \ldots, i_{\left.l+\frac{s-3}{2}\right\}} .\right.\end{cases} \\
& P_{2}= \begin{cases}u_{x} P^{-1} u_{x-\left(x_{1}-\frac{s+5}{2}\right)} & \text { if } x_{1}-\frac{s+3}{2} \leq x \leq x_{1}-1 \\
u_{x} P u_{x+\left(x_{1}-\frac{s+5}{2}\right)} & 1 \leq x \leq \frac{s+3}{2}\end{cases}
\end{aligned}
$$

Then, $P^{\prime}=P_{1} u_{x_{1}} P u_{l+1} P_{2}$ is a heterochromatic path of length $l+1$, a contradiction to the choice of $P$.

Case 2.2.2 If $k \equiv 4(\bmod 5)$, then $(4 l-2 k)-(k-l+2)=5 l-3 k-2=1$ which implies $k_{0}=s=2 l-k, x_{1}-2 l+k-1=x_{1}-s-1$, and $t_{2}=k-l-s=s+(k-l-2 s)=$ $s+(3 k-5 l)=s-3 \geq 0$.

We first consider the case when $t_{2}=s-3=0$, which implies that $k=9, l=6$, $k_{0}=s=3$ and $4=s+1 \leq x_{1} \leq l-s+1=4$. If there exists a $v \notin\left\{u_{4}, u_{5}\right\}$ such that $u_{7} v$ has some color not in $\left\{i_{3}, \ldots, i_{9}\right\}$, then $v \notin\left\{u_{4}, u_{5}, u_{6}, v_{1}, v_{2}, v_{3}\right\}$ and $P^{\prime}=v_{1} v_{2} v_{3} u_{4} u_{5} u_{6} u_{7} v$ is a heterochromatic path of length $l+1$, a contradiction. So, $i_{7} \in\left\{u_{1} u_{7}, u_{2} u_{7}, u_{3} u_{7}\right\}$. Let

$$
P^{\prime}= \begin{cases}v_{2} v_{3} u_{4} u_{5} u_{6} u_{7} u_{1} u_{2} & \text { if } u_{1} u_{7} \text { has color } i_{7} ; \\ v_{2} v_{2} u_{4} u_{5} u_{6} u_{7} u_{x} u_{x-1} & \text { if } u_{x} u_{7}(2 \leq x \leq 3) \text { has color } i_{7} .\end{cases}
$$

Then, $P^{\prime}$ is a heterochromatic path of length $l+1$, a contradiction.

Next we consider the case when $t_{2}>0$. Since $s \geq 4$, we have that $1<\left\lceil\frac{s}{2}\right\rceil \leq s-2$ which implies that $\left\{1,\left\lceil\frac{s}{2}\right\rceil\right\} \in\left\{y_{1}, \ldots, y_{t_{2}}\right\}$. Since $\left|\left\{\left\lfloor\frac{s}{2}\right\rfloor+2, \ldots, x_{1}-\left\lceil\frac{s}{2}\right\rceil-1\right\}\right|=x_{1}-$ $\left\lceil\frac{s}{2}\right\rceil-\left\lfloor\frac{s}{2}\right\rfloor-2=x_{1}-s-2$, there exists some $x \in\left\{1,2, \ldots,\left\lfloor\frac{s}{2}\right\rfloor+1\right\} \cup\left\{x_{1}-\left\lceil\frac{s}{2}\right\rceil, \ldots, x_{1}-1\right\}$ such that $u_{x} u_{l+1}$ has a color in $\left\{i_{l+s-\left\lceil\frac{x_{1}-1}{2}\right\rceil+1}, \ldots, i_{l+\left\lceil\frac{s+x_{1}-t_{2}}{2}\right\rceil-2}\right\}$. Let

$$
\begin{aligned}
& P_{1}= \begin{cases}v_{\left\lceil\frac{s}{2}\right\rceil} P^{-1} v_{1} v_{s} & \text { if } 1 \in\left\{y_{1}, \ldots, y_{t_{2}}\right\} \text { and } u_{x} u_{l+1} \text { has a color in } \\
& \left\{i_{l+\left\lceil\frac{s}{2}\right\rceil}, \ldots, i_{l+\left\lceil\frac{s+x_{1}-t_{2}}{2}\right\rceil-2}\right\} ; \\
v_{1} P v_{\left\lceil\frac{s}{2}\right\rceil} v_{s} \quad & \text { if }\left\lceil\frac{s}{2}\right\rceil \in\left\{y_{1}, \ldots, y_{t_{2}}\right\} \text { and } u_{x} u_{l+1} \text { has a color in } \\
& \left\{i_{l+\left\lceil\frac{s}{2}\right\rceil}, \ldots, i_{l+\left\lceil\frac{s+x_{1}-t_{2}}{2}\right\rceil-2}\right\} . \\
v_{\left\lceil\frac{s}{2}\right\rceil} P v_{s} & \text { if } u_{x} u_{l+1} \text { has a color in } \\
& \left\{i_{l+s-\left\lceil\frac{x_{1}-1}{2}\right\rceil+1}, \ldots, i_{l+\left\lceil\frac{s}{2}\right\rceil-1}\right\} .\end{cases} \\
& P_{2}= \begin{cases}u_{x} P^{-1} u_{x-\left(x_{1}-\left\lfloor\frac{s}{2}\right\rfloor-2\right)} & x_{1}-\left\lceil\frac{s}{2}\right\rceil \leq x \leq x_{1}-1 ; \\
u_{x} P u_{x+\left(x_{1}-\left\lfloor\frac{s}{2}\right\rfloor-2\right)} & 1 \leq x \leq\left\lfloor\frac{s}{2}\right\rfloor+1 .\end{cases}
\end{aligned}
$$

Then, since $\left(x_{1}-\left\lceil\frac{s}{2}\right\rceil\right)-\left(x_{1}-\left\lfloor\frac{s}{2}\right\rfloor-2\right) \geq 1$ and $\left(\left\lfloor\frac{s}{2}\right\rfloor+1\right)+\left(x_{1}-\left\lfloor\frac{s}{2}\right\rfloor-2\right)=x_{1}-1$, $P^{\prime}=P_{1} u_{x_{1}} P u_{l+1} P_{2}$ is a heterochromatic path of length at least $l+1$, a contradiction to the choice of $P$. 
Case $3 t_{1}=0$.

Since $t_{1}=0$, we know by Lemma 3.5 that $k \equiv 2,4(\bmod 5), k_{0}=s=2 l-k$ and $t_{2}=s-2$ if $k \equiv 4(\bmod 5) ; t_{2} \geq s-3$ if $k \equiv 2(\bmod 5)$, and there are exactly $l-1$ different colors not in $\left\{i_{k_{0}}, i_{k_{0}+1}, \ldots, i_{l}\right\}$ that belong to $C\left(u_{1} u_{l+1}, u_{2} u_{l+1}, \ldots, u_{l-1} u_{l+1}\right)$. On the other hand, by Lemma 3.11 we have that $i_{1} \notin C\left(u_{1} v_{s}, u_{2} v_{s}, \ldots, u_{l} v_{s}\right)$, and by Lemma $3.5 C N\left(v_{s}\right)-C\left(\left\{u_{s+1} v_{s}, \ldots, u_{l+1} v_{s}, v_{1} v_{s}, \ldots, v_{s-2} v_{s}\right\}\right) \subseteq\left\{i_{k_{0}}, \ldots, i_{l+s-1}\right\}$. Since $d^{c}\left(v_{s}\right) \geq k$ and if $k \equiv 4(\bmod 5), t_{2}=s-2 ;$ if $k \equiv 2(\bmod 5), t_{2} \geq s-3$, which implies that $k \equiv 2(\bmod 5), t_{2}=s-2$ or $i_{1} \in\left\{v_{1} v_{s}, \ldots, v_{s-2} v_{s}\right\}$ and $i_{2} \in\left\{u_{s+1} v_{s}, \ldots, u_{l} v_{s}\right\}$, $i_{3} \in\left\{u_{s+1} v_{s}, \ldots, u_{l} v_{s}\right\}$ if $s \geq 4$.

Since $k \geq 8$, we consider the case when $s \geq 4$. If there exists an $x \in\{l-s+4, \ldots, l\}$ such that $u_{x} u_{l+1}$ has color $i_{2}$, then let $P^{\prime}=v_{1} P v_{s} u_{x} P^{-1} u_{x-(l+2-s)+1}$. Since $l-s+4 \leq x \leq l$, we have $x-(l+2-s)+1 \geq(l-s+4)-(l-s+2)+1=3$, and so $P^{\prime}$ is a heterochromatic path of length $l+1$, a contradiction to the choice of $P$. So, $i_{2} \in C\left(\left\{u_{s+1} v_{s}, \ldots, u_{l-s+3} v_{s}\right\}\right)$.

If there exists an $3 \leq x^{\prime} \leq x-1$ such that $u_{x^{\prime}} u_{l+1}$ has a color not in $\left\{i_{2}, i_{3}, \ldots, i_{l+s-1}\right\}$, then let

$$
P^{\prime}= \begin{cases}v_{1} P v_{s} u_{x} P u_{l+1} u_{x^{\prime}} P^{-1} u_{x^{\prime}-(x-s)+1} & \text { if } x-s+2 \leq x^{\prime} \leq x-1 \\ v_{1} P v_{s} u_{x} P u_{l+1} u_{x^{\prime}} P u_{x^{\prime}+(x-s)-1} & \text { if } 3 \leq x^{\prime} \leq x-s+1\end{cases}
$$

Note that if $3 \leq x^{\prime} \leq x-s+1$, then $x^{\prime}+(x-s)-1 \leq x+(x-2 s) \leq x+(l-s+3-2 s)=$ $x+(l-6 l+3 k+3)=x-1$, and so $P^{\prime}$ is a heterochromatic path of length $l+1$, a contradiction to the choice of $P$. So, $C\left(\left\{u_{3} u_{l+1}, \ldots, u_{x-1} u_{l+1}\right\}\right) \subseteq\left\{i_{2}, i_{3}, \ldots, i_{l+s-1}\right\}$.

We first consider the case when $x=s+1$. If there exists an $1 \leq x^{\prime} \leq x-1$ such that $u_{x^{\prime}} u_{l+1}$ has a color in $\left\{i_{3}, \ldots, i_{k_{0}-1}\right\}$, then $P^{\prime}=v_{1} P v_{s} u_{x} P u_{l+1} u_{x^{\prime}}$ is a heterochromatic path of length $l+1$, a contradiction to the choice of $P$. So, there are $x-1-1=x-2=s-1$ colors in $\left\{i_{l+1}, \ldots, i_{l+s-1}\right\}$ that belong to $C\left(\left\{u_{1} u_{l+1}, \ldots, u_{x-1} u_{l+1}\right\}\right)$. Then there must exist an $1 \leq x^{\prime} \leq x-1$ such that $u_{x^{\prime}} u_{l+1}$ has color $i_{l+s-1}$, and so $P^{\prime}=v_{s-1} P^{-1} v_{1} v_{s} u_{s+1} P u_{l+1} u_{x^{\prime}}$ is a heterochromatic path of length $l+1$, a contradiction to the choice of $P$.

Next we consider the case when $x \geq s+2$. There are at least $(x-3)-\left(k_{0}-2\right)=x-k_{0}-$ $1=x-s-1>0$ colors in $\left\{i_{l+1}, \ldots, i_{l+s-1}\right\}$ that belong to $C\left(\left\{u_{3} u_{l+1}, \ldots, u_{x-1} u_{l+1}\right\}\right)$. If there exists an $3 \leq x^{\prime} \leq x-1$ such that $u_{x^{\prime}} u_{l+1}$ has a color in $\left\{i_{l+1}, \ldots, i_{l+s-\left\lceil\frac{x+1}{2}\right\rceil}\right.$, $\left.i_{l+\left\lceil\frac{x+1}{2}\right\rceil-1}, \ldots, i_{l+s-1}\right\}$, then by Lemma 3.8 there is a heterochromatic path of length $l+$ 1 , a contradiction to the choice of $P$. So, $C\left(\left\{u_{3} u_{l+1}, \ldots, u_{x-1} u_{l+1}\right\}\right) \subseteq\left\{i_{2}, \ldots, i_{k_{0}-1}\right.$, $\left.i_{l+s-\left\lceil\frac{x+1}{2}\right\rceil+1}, \ldots, i_{l+\left\lceil\frac{x+1}{2}\right\rceil-2}\right\}$. Then, we consider the color $i_{3}$. If there exists a $z \in\{1, \ldots, s$, $l-s+5, \ldots, l-1\}$ such that $u_{z} v_{s}$ has color $i_{3}$, then let

$$
P^{\prime}= \begin{cases}u_{s+1} P v_{s} u_{z} & \text { if } 1 \leq z \leq s \\ v_{1} P v_{s} u_{z} P^{-1} u_{z-(l+2-s)+1} & \text { if } l-s+5 \leq z \leq l-1 .\end{cases}
$$

So, $P^{\prime}$ is a heterochromatic path of length $l+1$, a contradiction to the choice of $P$. Since $k_{0}=s \geq 4$, we have $i_{3} \in C\left(\left\{u_{1} v_{s}, \ldots, u_{l} v_{s}\right\}\right)$, and so there exists a $s+1 \leq z \leq l-s+4$ such that $u_{z} v_{s}$ has color $i_{3}$. Then we consider the following subcases:

Case $3.1 i_{2}, i_{3} \in C\left(\left\{u_{3} u_{l+1}, \ldots, u_{x-1} u_{l+1}\right\}\right)$.

First we consider the case when there exists an $4 \leq x^{\prime} \leq x-1$ such that $u_{x^{\prime}} u_{l+1}$ has 
color $i_{3}$.

If $x=l-s+3$ and $x^{\prime}=s+1$, since there are at least $(x-3)-\left(k_{0}-2\right)=x-s-1=$ $l-2 s+2=s+(l-3 s+2)=s+(l-6 l+3 k+2)=s-2$ colors in $\left\{i_{l+1}, \ldots, i_{l+s-1}\right\}$ that belong to $C\left(\left\{u_{3} u_{l+1}, \ldots, u_{x-1} u_{l+1}\right\}\right)$, then there exists an $x^{\prime \prime} \in\{3, \ldots, s-1, s+2, \ldots, x-1\}$ such that $u_{x^{\prime \prime}} u_{l+1}$ has a color in $\left\{i_{l+1}, i_{l+s-2}, i_{l+s-1}\right\}$. Let

$$
\begin{aligned}
P_{1} & = \begin{cases}v_{2} P v_{s} & \text { if } u_{x^{\prime \prime}} u_{l+1} \text { has color } i_{l+1} ; \\
v_{s-2} P^{-1} v_{1} v_{s} & \text { if } u_{x^{\prime \prime}} u_{l+1} \text { has color } i_{l+s-2} \text { or } i_{l+s-1} . \\
P_{1} u_{x} P u_{l+1} u_{x^{\prime \prime}} P^{-1} u_{x^{\prime \prime}-(x-s)} & \text { if } s+2 \leq x^{\prime \prime} \leq x-1 \\
P_{1} u_{x} P u_{l+1} u_{x^{\prime \prime}} P u_{x^{\prime \prime}+(x-s)} & \text { if } 3 \leq x^{\prime \prime} \leq s-1 .\end{cases}
\end{aligned}
$$

Note that if $s+2 \leq x^{\prime \prime} \leq x-1$, then $x^{\prime \prime}-(x-s) \geq 2 s-x+2=2 s-l+s-3+2=$ $3(2 l-k)-l-1=5 l-3 k-1=3$; if $3 \leq x^{\prime \prime} \leq s-1$, then $x^{\prime \prime}+(x-s) \leq x-1$. So, $P^{\prime}$ is a heterochromatic path of length $l+1$, a contradiction to the choice of $P$.

Otherwise, $x \leq l-s+2$ or $x=l-s+3, x^{\prime} \neq s+1$. Let

$$
P^{\prime}= \begin{cases}v_{1} P v_{s} u_{x} P u_{l+1} u_{x^{\prime}} P^{-1} u_{x^{\prime}-(x-s)+1} & \text { if } x-s+3 \leq x^{\prime} \leq x-1 \\ v_{1} P v_{s} u_{x} P u_{l+1} u_{x^{\prime}} P u_{x^{\prime}+(x-s)-1} & \text { if } 4 \leq x^{\prime} \leq x-s+2\end{cases}
$$

Note that if $x \leq l-s+2$, then $x-s+2 \leq l-s+2-s+2=l-2 s+4=s+$ $(l-3 s+4)=s+(3 k-5 l+4)=s$, and if $x=l-s+3$ and $x^{\prime} \neq s+1$, then $x-s+2=l-s+3-s-2=l-2 s+5=s+1$. So, if $4 \leq x^{\prime} \leq x-s+2$, then $x^{\prime} \leq s$ and hence $x^{\prime}+(x-s)-1 \leq x-1$, and so $P^{\prime}$ is a heterochromatic path of length $l+1$, a contradiction to the choice of $P$.

Now we consider the case when $u_{3} u_{l+1}$ has color $i_{3}$. Then there exists an $4 \leq x^{\prime} \leq x-1$ such that $u_{x^{\prime}} u_{l+1}$ has color $i_{2}$. Let

$$
P^{\prime}= \begin{cases}v_{1} P v_{s} u_{z} P u_{l+1} u_{x^{\prime}} P^{-1} u_{x^{\prime}-(z-s)+1} & \text { if } z-s+3 \leq x^{\prime} \leq x-1 \\ v_{1} P v_{s} u_{z} P u_{l+1} u_{x^{\prime}} P u_{x^{\prime}+(z-s)-1} & \text { if } 4 \leq x^{\prime} \leq \min \{z-s+2, s\} .\end{cases}
$$

Then, $P^{\prime}$ is a heterochromatic path of length $l+1$, a contradiction to the choice of $P$. So, $s+1 \leq x^{\prime} \leq z-s+2$, which implies $z \geq 2 s-1$. On the other hand, $z \leq l-s+4=$ $2 s+(l-3 s+4)=2 s, 2 s-1 \leq z \leq 2 s$. So we distinguish two cases: $z=2 s-1$, then $s+1 \leq x^{\prime} \leq z-s+2=s+1$; and $z=2 s$, then $s+1 \leq x^{\prime} \leq z-s+2=s+2$.

Case 3.1.1 $z=2 s-1, x^{\prime}=s+1$. If there exists an $4 \leq x^{\prime \prime} \leq z-1$ such that $u_{x^{\prime \prime}} u_{l+1}$ has a color not in $\left\{i_{3}, \ldots, i_{k_{0}-1}, i_{l+1}, \ldots, i_{l+s-1}\right\}$, then $x^{\prime \prime} \neq s+1$. Let

$$
P^{\prime}= \begin{cases}v_{1} P v_{s} u_{z} P u_{l+1} u_{x^{\prime \prime}} P^{-1} u_{x^{\prime \prime}-(z-s)+1} & \text { if } s+2 \leq x^{\prime \prime} \leq z-1 \\ v_{1} P v_{s} u_{z} P u_{l+1} u_{x^{\prime \prime}} P u_{x^{\prime \prime}+(z-s)-1} & \text { if } 4 \leq x^{\prime \prime} \leq s\end{cases}
$$

Note that if $s+2 \leq x^{\prime \prime} \leq z-1$, then $x^{\prime \prime}-(z-s)+1 \geq s+2-(z-s)+1=4$, and so $P^{\prime}$ is a heterochromatic path of length $l+1$, a contradiction to the choice of $P$. So, there are at least $(z-4)-\left(k_{0}-3\right)=z-s-1=s-2$ colors in $\left\{i_{l+1}, \ldots, i_{l+s-1}\right\}$ that belong to $C\left(\left\{u_{4} u_{l+1}, \ldots, u_{z-1} u_{l+1}\right\}\right)$, and there are at least 2 colors in $\left\{i_{l+1}, i_{l+s-2}, i_{l+s-1}\right\}$ that 
belong to $C\left(\left\{u_{4} u_{l+1}, \ldots, u_{s} u_{l+1}, u_{s+2} u_{l+1}, \ldots, u_{z-1} u_{l+1}\right\}\right)$. If there exists an $4 \leq x^{\prime \prime} \leq z-1$ such that $u_{x^{\prime \prime}} u_{l+1}$ has color $i_{l+s-1}$, then $x^{\prime \prime} \neq s+1$. Let

$$
P^{\prime}= \begin{cases}v_{s-1} P^{-1} v_{1} v_{s} u_{z} P u_{l+1} u_{x^{\prime \prime}} P^{-1} u_{x^{\prime \prime}-(z-s)+1} & \text { if } s+2 \leq x^{\prime \prime} \leq z-1 \\ v_{s-1} P^{-1} v_{1} v_{s} u_{z} P u_{l+1} u_{x^{\prime \prime}} P u_{x^{\prime \prime}+(z-s)-1} & \text { if } 4 \leq x^{\prime \prime} \leq s\end{cases}
$$

Then, $P^{\prime}$ is a heterochromatic path of length $l+1$, a contradiction to the choice of $P$. So, $i_{l+s-1} \notin C\left(\left\{u_{4} u_{l+1}, \ldots, u_{z-1} u_{l+1}\right\}\right)$ and $i_{l+1}, i_{l+s-2} \in\left\{u_{4} u_{l+1}, \ldots, u_{z-1} u_{l+1}\right\}$. If there exists an $4 \leq x^{\prime \prime} \leq z-1, x^{\prime \prime} \neq s, s+1, s+2$ such that $u_{x^{\prime \prime}} u_{l+1}$ has color $i_{l+1}$ or $i_{l+s-2}$, then let

$$
P_{1}= \begin{cases}v_{2} P v_{s} & \text { if } u_{x^{\prime \prime}} u_{l+1} \text { has color } i_{l+1} ; \\ v_{s-2} P^{-1} v_{1} v_{s} & \text { if } u_{x^{\prime \prime}} u_{l+1} \text { has color } i_{l+s-2} . \\ P_{1} u_{z} P u_{l+1} u_{x^{\prime \prime}} P^{-1} u_{x^{\prime \prime}-(z-s)} & \text { if } s+3 \leq x^{\prime \prime} \leq z-1 ; \\ P_{1} u_{z} P u_{l+1} u_{x^{\prime \prime}} P u_{x^{\prime \prime}+(z-s)} & \text { if } 4 \leq x^{\prime \prime} \leq s-1 .\end{cases}
$$

So, $P^{\prime}$ is a heterochromatic path of length $l+1$, a contradiction to the choice of $P$. So, $C\left(\left\{u_{s} u_{l+1}, u_{s+2} u_{l+1}\right\}\right)=\left\{i_{l+1}, i_{l+s-2}\right\}$. Since $x \geq s+2$, we consider the case when $x \geq s+3$, first. Let

$$
P^{\prime}= \begin{cases}v_{2} P v_{s} u_{x} P u_{l+1} u_{s+2} P^{-1} u_{2 s-x+2} & \text { if } u_{s+2} u_{l+1} \text { has color } i_{l+1} ; \\ v_{s-2} P^{-1} v_{1} v_{s} u_{x} P u_{l+1} u_{s+2} P^{-1} u_{2 s-x+2} & \text { if } u_{s+2} u_{l+1} \text { has color } i_{l+s-2} .\end{cases}
$$

Since $2 s-x+2 \geq 2 s-(l-s+3)+2=6 l-3 k-l-1=3, P^{\prime}$ is a heterochromatic path of length $l+1$, a contradiction to the choice of $P$. Then $x=s+2$. Let

$$
P^{\prime}= \begin{cases}v_{2} P v_{s} u_{s+2} P u_{l+1} u_{s} u_{s-1} u_{s-2} & \text { if } u_{s} u_{l+1} \text { has color } i_{l+1} ; \\ v_{s-2} P^{-1} v_{1} v_{s} u_{s+2} P u_{l+1} u_{s} u_{s-1} u_{s-2} & \text { if } u_{s} u_{l+1} \text { has color } i_{l+s-2} .\end{cases}
$$

If $s \geq 5, P^{\prime}$ is a heterochromatic path of length $l+1$, a contradiction to the choice of $P$. So, $s=4$. Since there are exactly $l-1$ different colors not in $\left\{i_{k_{0}}, i_{k_{0}+1}, \ldots, i_{l}\right\}$ that belong to $C\left(\left\{u_{1} u_{l+1}, \ldots, u_{l-1} u_{l+1}\right\}\right), v_{1} P v_{s} u_{s+2} P u_{l+1} u_{1} u_{2}$ is a heterochromatic path of length $l+1$ if $C\left(u_{1} u_{l+1}\right) \notin\left\{i_{1}, \ldots, i_{l+s-1}\right\} ; v_{1} P v_{s} u_{s+2} P u_{l+1} u_{2} u_{1}$ is a heterochromatic path of length $l+1$ if $C\left(u_{2} u_{l+1}\right) \notin\left\{i_{1}, \ldots, i_{l+s-1}\right\}$. Then $u_{1} u_{l+1}$ or $u_{2} u_{l+1}$ has color $i_{l+s-1}$ and $\{1,2\} \cap\left\{y_{1}, \ldots, y_{t_{2}}\right\} \neq \emptyset$. If $u_{1} u_{l+1}$ has color $i_{l+s-1}$, then let

$$
P^{\prime}=\left\{\begin{array}{ll}
u_{s-1} P^{-1} u_{1} u_{l+1} v_{1} v_{2} v_{s} u_{z} P^{-1} u_{s+1} & \text { if } 2 \in\left\{y_{1}, \ldots, y_{t_{2}}\right\} \\
v_{s-1} P^{-1} v_{1} v_{s} u_{s+2} P u_{l+1} u_{1} u_{2} & \text { if } 1 \in\left\{y_{1}, \ldots, y_{t_{2}}\right\}
\end{array} .\right.
$$

So, $P^{\prime}$ is a heterochromatic path of length $l+1$, a contradiction to the choice of $P$. So, $u_{2} u_{l+1}$ has color $i_{l+s-1}$. Let

$$
P^{\prime}= \begin{cases}v_{3} v_{2} v_{1} v_{s} u_{s+2} P u_{l+1} u_{2} u_{1} & \text { if } 1 \in\left\{y_{1}, \ldots, y_{t_{2}}\right\} \\ u_{1} u_{2} u_{l+1} u_{s} P u_{z} v_{s} v_{2} v_{3} & \text { if } 2 \in\left\{y_{1}, \ldots, y_{t_{2}}\right\} \text { and } u_{s} u_{l+1} \text { has color } i_{l+1} ; \\ u_{1} u_{2} u_{l+1} u_{s} P u_{z} v_{s} v_{2} v_{1} & \text { if } 2 \in\left\{y_{1}, \ldots, y_{t_{2}}\right\} \text { and } u_{s} u_{l+1} \text { has color } i_{l+s-2}\end{cases}
$$

Then, $P^{\prime}$ is a heterochromatic path of length $l+1$, a contradiction to the choice of $P$.

Case 3.1.2 $z=2 s, x^{\prime}=s+1$ or $s+2$. If there exists an $4 \leq x^{\prime \prime} \leq z-1, x^{\prime \prime} \neq s+1, s+2$ such that $u_{x^{\prime \prime}} u_{l+1}$ has a color not in $\left\{i_{3}, \ldots, i_{l+s-1}\right\}$, then let

$$
P^{\prime}= \begin{cases}v_{1} P v_{s} u_{z} P u_{l+1} u_{x^{\prime \prime}} P^{-1} u_{x^{\prime \prime}-(z-s)+1} & \text { if } s+3 \leq x^{\prime \prime} \leq z-1 \\ v_{1} P v_{s} u_{z} P u_{l+1} u_{x^{\prime \prime}} P u_{x^{\prime \prime}+(z-s)-1} & \text { if } 4 \leq x^{\prime \prime} \leq s\end{cases}
$$


Note that if $s+3 \leq x^{\prime \prime} \leq z-1$, then $x^{\prime \prime}-(z-s)+1 \geq s+3-(z-s)+1=4$, and so $P^{\prime}$ is a heterochromatic path of length $l+1$, a contradiction to the choice of $P$. So, there are at least $(z-4-1)-\left(k_{0}-3\right)=z-s-2=s-2$ colors in $\left\{i_{l+1}, \ldots, i_{l+s-1}\right\}$ that belong to $C\left(\left\{u_{4} u_{l+1}, \ldots, u_{s} u_{l+1}, u_{s+3} u_{l+1}, \ldots, u_{z-1} u_{l+1}\right\}\right)$, and there are at least 2 colors in $\left\{i_{l+1}, i_{l+s-2}, i_{l+s-1}\right\}$ that belong to $C\left(\left\{u_{4} u_{l+1}, \ldots, u_{s} u_{l+1}, u_{s+3} u_{l+1}, \ldots, u_{z-1} u_{l+1}\right\}\right)$. If there exists an $4 \leq x^{\prime \prime} \leq z-1, x^{\prime \prime} \neq s+1, s+2$ such that $u_{x^{\prime \prime}} u_{l+1}$ has color $i_{l+s-1}$, then let

$$
P^{\prime}= \begin{cases}v_{s-1} P^{-1} v_{1} v_{s} u_{z} P u_{l+1} u_{x^{\prime \prime}} P^{-1} u_{x^{\prime \prime}-(z-s)+1} & \text { if } s+3 \leq x^{\prime \prime} \leq z-1 ; \\ v_{s-1} P^{-1} v_{1} v_{s} u_{z} P u_{l+1} u_{x^{\prime \prime}} P u_{x^{\prime \prime}+(z-s)-1} & \text { if } 4 \leq x^{\prime \prime} \leq s\end{cases}
$$

So, $P^{\prime}$ is a heterochromatic path of length $l+1$, a contradiction to the choice of $P$. So, $i_{l+s-1} \notin C\left(\left\{u_{4} u_{l+1}, \ldots, u_{s} u_{l+1}, u_{s+3} u_{l+1}, \ldots, u_{z-1} u_{l+1}\right\}\right), i_{l+1}, i_{l+s-2} \in\left\{u_{4} u_{l+1}, \ldots\right.$, $\left.u_{s} u_{l+1}, u_{s+3} u_{l+1}, \ldots, u_{z-1} u_{l+1}\right\}$. If there exists an $4 \leq x^{\prime \prime} \leq z-1, x^{\prime \prime} \neq s, s+1, s+2, s+3$ such that $u_{x^{\prime \prime}} u_{l+1}$ has color $i_{l+1}$ or $i_{l+s-2}$, then let

$$
P_{1}= \begin{cases}v_{2} P v_{s} & \text { if } u_{x^{\prime \prime}} u_{l+1} \text { has color } i_{l+1} ; \\ v_{s-2} P^{-1} v_{1} v_{s} & \text { if } u_{x^{\prime \prime}} u_{l+1} \text { has color } i_{l+s-2} \\ P_{1} u_{z} P u_{l+1} u_{x^{\prime \prime}} P^{-1} u_{x^{\prime \prime}-(z-s)} & \text { if } s+4 \leq x^{\prime \prime} \leq z-1 ; \\ P_{1} u_{z} P u_{l+1} u_{x^{\prime \prime}} P u_{x^{\prime \prime}+(z-s)} & \text { if } 4 \leq x^{\prime \prime} \leq s-1 .\end{cases}
$$

So, $P^{\prime}$ is a heterochromatic path of length $l+1$, a contradiction to the choice of $P$. So, $C\left(\left\{u_{s} u_{l+1}, u_{s+3} u_{l+1}\right\}\right)=\left\{i_{l+1}, i_{l+s-2}\right\}$. Since $x \geq s+2$, we consider the case when $x \geq s+4$, first. Let

$$
P^{\prime}=\left\{\begin{array}{ll}
v_{2} P v_{s} u_{x} P u_{l+1} u_{s+3} P^{-1} u_{2 s-x+3} & \text { if } u_{s+3} u_{l+1} \text { has color } i_{l+1} ; \\
v_{s-2} P^{-1} v_{1} v_{s} u_{x} P u_{l+1} u_{s+3} P^{-1} u_{2 s-x+3} & \text { if } u_{s+3} u_{l+1} \text { has color } i_{l+s-2} .
\end{array} .\right.
$$

Since $2 s-x+3 \geq 2 s-(l-s+3)+3=6 l-3 k-l=4, P^{\prime}$ is a heterochromatic path of length $l+1$, a contradiction to the choice of $P$. Then we consider the case when $x=s+2$. Let

$$
P^{\prime}= \begin{cases}v_{2} P v_{s} u_{s+2} P u_{l+1} u_{s} u_{s-1} u_{s-2} & \text { if } u_{s} u_{l+1} \text { has color } i_{l+1} ; \\ v_{s-2} P^{-1} v_{1} v_{s} u_{s+2} P u_{l+1} u_{s} u_{s-1} u_{s-2} & \text { if } u_{s} u_{l+1} \text { has color } i_{l+s-1} .\end{cases}
$$

If $s \geq 5$, then $P^{\prime}$ is a heterochromatic path of length $l+1$, a contradiction to the choice of $P$, and so $s=4$. Since $C\left(\left\{u_{3} u_{l+1}, \ldots, u_{x-1} u_{l+1}\right\}\right) \subseteq\left\{i_{2}, \ldots, i_{l+s-1}\right\}, u_{3} u_{l+1}$ has color $i_{3}$, and if $u_{1} u_{l+1}$ has a color not in $\left\{i_{1}, i_{2}, \ldots, i_{l+s-1}\right\}$, then $P^{\prime}=v_{1} P v_{s} u_{s+2} P u_{l+1} u_{1} u_{2}$ is a heterochromatic path of length $l+1$; if $u_{2} u_{l+1}$ has a color not in $\left\{i_{1}, i_{2}, \ldots, i_{l+s-1}\right\}$, then $P^{\prime}=v_{1} P v_{s} u_{s+2} P u_{l+1} u_{2} u_{1}$ is a heterochromatic path of length $l+1$, a contradiction to the choice of $P$. So, $u_{s+2} u_{l+1}$ has a color not in $\left\{i_{1}, i_{2}, \ldots, i_{l+s-1}\right\}, u_{s+1} u_{l+1}$ has color $i_{2}$, $u_{1} u_{l+1}$ or $u_{2} u_{l+1}$ has color $i_{l+s-1}$ and $\{1,2\} \cap\left\{y_{1}, \ldots, y_{t_{2}}\right\} \neq \emptyset$. If $u_{1} u_{l+1}$ has color $i_{l+s-1}$, then let

$$
P^{\prime}= \begin{cases}u_{s-1} P^{-1} u_{1} u_{l+1} v_{1} v_{2} v_{s} u_{z} P^{-1} u_{s+2} & \text { if } 2 \in\left\{y_{1}, y_{2}, \ldots, y_{t_{2}}\right\} \\ v_{s-1} P^{-1} v_{1} v_{s} u_{s+2} P u_{l+1} u_{1} u_{2} & \text { if } 1 \in\left\{y_{1}, \ldots, y_{t_{2}}\right\} .\end{cases}
$$

So, $P^{\prime}$ is a heterochromatic path of length $l+1$, a contradiction to the choice of $P$. So, $u_{2} u_{l+1}$ has color $i_{l+s-1}$. Let

$$
P^{\prime}= \begin{cases}u_{1} u_{2} u_{l+1} u_{s} P u_{z} v_{s} v_{2} & \text { if } 2 \in\left\{y_{1}, \ldots, y_{t_{2}}\right\} \\ v_{s-1} P^{-1} v_{1} v_{s} u_{s+2} P u_{l+1} u_{2} u_{1} & \text { if } 1 \in\left\{y_{1}, \ldots, y_{t_{2}}\right\}\end{cases}
$$


Then, $P^{\prime}$ is a heterochromatic path of length $l+1$, a contradiction to the choice of $P$.

Last, we consider the case when $x=s+3$. Since $C\left(\left\{u_{3} u_{l+1}, \ldots, u_{x-1} u_{l+1}\right\}\right) \subseteq$ $\left\{i_{2}, \ldots, i_{l+s-1}\right\}$, we have that $C\left(\left\{u_{s+1} u_{l+1}, u_{s+2} u_{l+1}\right\}\right)=\left\{i_{2}, i_{l+s-1}\right\}$. Let

$$
P^{\prime}= \begin{cases}v_{s-1} P^{-1} v_{1} v_{s} u_{s+3} P u_{l+1} u_{s+2} u_{s+1} u_{s} & \text { if } u_{s+2} u_{l+1} \text { has color } i_{l+s-1} ; \\ v_{s-1} P^{-1} v_{1} v_{s} u_{s+3} P u_{l+1} u_{s+1} u_{s} u_{s-1} & \text { if } u_{s+1} u_{l+1} \text { has color } i_{l+s-1} .\end{cases}
$$

Then, $P^{\prime}$ is a heterochromatic path of length $l+1$, a contradiction to the choice of $P$.

Case $3.2\left\{i_{2}, i_{3}\right\} \nsubseteq C\left(\left\{u_{3} u_{l+1}, \ldots, u_{x-1} u_{l+1}\right\}\right)$. Since $\mid\left(\left\{i_{4}, \ldots, i_{k_{0}-1}, i_{l+s-\left\lceil\frac{x+1}{2}\right\rceil+1}\right.\right.$,

$\left.\ldots, i_{l+\left\lceil\frac{x+1}{2}\right\rceil-2}\right\} \mid+1=2\left\lceil\frac{x+1}{2}\right\rceil-5 \leq x+2-5=x-3$, we have that $\left\lceil\frac{x+1}{2}\right\rceil=\frac{x+2}{2}$ and $\left\{i_{l+s-\left\lceil\frac{x+1}{2}\right\rceil+1}, \ldots, i_{l+\left\lceil\frac{x+1}{2}\right\rceil-2}\right\} \subseteq C\left(\left\{u_{3} u_{l+1}, \ldots, u_{x-1} u_{l+1}\right\}\right)$. Then, there exists an $x^{\prime} \in$ $\left\{3, \ldots, \frac{x}{2}, \frac{x}{2}+2, \ldots, x-1\right\}$ such that $u_{x^{\prime}} u_{l+1}$ has color $i_{l+s-\left\lceil\frac{x+1}{2}\right\rceil+1}$ or $i_{l+\left\lceil\frac{x+1}{2}\right\rceil-2}$. Let

$$
\begin{aligned}
& P_{1}= \begin{cases}v_{s-\left\lceil\frac{x+1}{2}\right\rceil+2} P v_{s} & \text { if } u_{x^{\prime}} u_{l+1} \text { has color } i_{l+s-\left\lceil\frac{x+1}{2}\right\rceil+1} \\
v_{\left\lceil\frac{x+1}{2}\right\rceil-2} P^{-1} v_{1} v_{s} & \text { if } u_{x^{\prime}} u_{l+1} \text { has color } i_{l+\left\lceil\frac{x+1}{2}\right\rceil-2} .\end{cases} \\
& P^{\prime}= \begin{cases}P_{1} u_{x} P u_{l+1} u_{x^{\prime}} P^{-1} u_{x^{\prime}-\frac{x}{2}+1} & \text { if } \frac{x}{2}+2 \leq x^{\prime} \leq x-1 ; \\
P_{1} u_{x} P u_{l+1} u_{x^{\prime}} P u_{x^{\prime}+\frac{x}{2}-1} & \text { if } 3 \leq x^{\prime} \leq \frac{x}{2}\end{cases}
\end{aligned}
$$

Then, $P^{\prime}$ is a heterochromatic path of length $l+1$, a contradiction to the choice of $P$.

The proof is now complete.

\section{Concluding remarks}

Finally, we consider whether our lower bound is best possible. It is obvious that when $k=1,2$, the bound is best possible. Next we consider the case when $k \geq 3$.

Remark 5.1 For any integer $k \geq 3$, there is an edge-colored graph $G_{k}$ with $d^{c}(v) \geq k$ for all the vertices $v$ in $G$ such that any longest heterochromatic path of $G$ is of length $k-1$.

In fact, let $G_{k}$ be an edge-colored graph whose vertices are the ordered $(k-1)$-tuples of 0 's and 1's; two vertices are joined by an edge if and only if they differ in exactly one coordinate or they differ in all coordinates. An edge is in color $j(1 \leq j \leq k-1)$ if and only if its two ends differ in exactly the $j$ th coordinate, or in color $k$ if and only if its two ends differ in all the coordinates. Then it is not difficult to check that $G_{k}$ is a graph we want.

Another class of graphs is given as follows. Since $K_{k}$ is $(k-1)$-edge-colorable when $k$ is even, we can get a proper $k$-edge coloring for $K_{k+1}$ when $k$ is odd. Denote it by $G_{k}^{\prime}$. Then, it is obvious that the longest heterochromatic path in $G_{k}^{\prime}$ is of length $k-1$ when $k$ is odd.

Remark 5.2 Actually, we can show that for $1 \leq k \leq 5$ any graph $G$ under the color degree condition has a heterochromatic path of length at least $k$, with only one exceptional graph $K_{4}$ for $k=3$, one exceptional graph $G_{4}$ for $k=4$ and three exceptional graphs for $k=5$, for which $G$ has a heterochromatic path of length at least $k-1$. 
To show this, let us construct an edge-colored graph $H$, first. Let $H$ have the vertex set $\left\{u_{1}, u_{2}, u_{3}, u_{4}, u_{5}, u_{6}, u_{7}, u_{8}\right\}$. The edges of $H$ and their colors are given as follows: $C\left(\left\{u_{1} u_{2}, u_{3} u_{7}, u_{4} u_{8}, u_{5} u_{6}\right\}\right)=1, C\left(\left\{u_{1} u_{4}, u_{2} u_{3}, u_{5} u_{8}, u_{6} u_{7}\right\}\right)=2, C\left(\left\{u_{1} u_{5}, u_{2} u_{6}, u_{3} u_{4}\right.\right.$, $\left.\left.u_{7} u_{8}\right\}\right)=3, C\left(\left\{u_{1} u_{6}, u_{2} u_{5}, u_{3} u_{8}, u_{4} u_{7}\right\}\right)=4$, and $C\left(\left\{u_{1} u_{7}, u_{2} u_{8}, u_{3} u_{5}, u_{4} u_{6}\right\}\right)=5$. Then, $d^{c}(v)=5$ for every vertex $v \in V(H)$ and the longest heterochromatic path in $H$ is of length 4 .

Then, by exhausting all the possible adjacency and colorings of edges for $1 \leq k \leq 5$, we can get that any graph $G$ under the color degree condition has a heterochromatic path of length at least $k$, with only one exceptional graph $K_{4}$ for $k=3$, one exceptional graph $G_{4}$ for $k=4$ and three exceptional graphs $G_{5}, G_{5}^{\prime}$ and $H$ for $k=5$, for which $G$ has a heterochromatic path of length at least $k-1$, where $G_{k}$ and $G_{k}^{\prime}$ are given in the proof of Remark 5.1. When $k$ becomes larger, there might be more such exceptional graphs. The tedious details have to be omitted.

Now we know that our lower bound is best possible when $1 \leq k \leq 7$. But we still do not know whether it is best possible when $k \geq 8$. We have tried all the possible cases when $k=8$ in order to find a graph to show that our bound is best possible, but failed. To end this paper, we propose the following conjecture:

Conjecture 5.3 Let $G$ be an edge-colored graph and $k \geq 3$ an integer. Suppose that $d^{c}(v) \geq k$ for every vertex $v$ of $G$. Then $G$ has a heterochromatic path of length at least $k-1$.

From the examples above we know that if this conjecture is true, then it would be best possible. T. Jiang once told us that they showed that the conjecture is true for complete graphs.

Acknowledgement: This work was supported by NSFC. The authors would like to thank the referee for helpful suggestions and comments.

\section{References}

[1] M. Albert, A. Frieze and B. Reed, Multicolored Hamilton cycles, Electronic J. Combin. 2 (1995), Research Paper R10.

[2] M. Axenovich, T. Jiang and Zs. Tuza, Local anti-Ramsey numbers of graphs, Combin. Probab. Comput. 12 (2003), 495-511.

[3] J.A. Bondy and U.S.R. Murty, Graph Theory with Applications, Macmillan London and Elsevier, New York (1976).

[4] H.J. Broersma, X.L. Li, G. Woeginger and S.G. Zhang, Paths and cycles in colored graphs, Australasian J. Combin. 31 (2005), 297-309.

[5] W.S. Chou, Y. Manoussakis, O. Megalaki, M. Spyratos and Zs. Tuza, Paths through fixed vertices in edge-colored graphs, Math. Inf. Sci. Hun. 32 (1994), 49-58. 
[6] P. Erdős and Zs. Tuza, Rainbow Hamiltonian paths and canonically colored subgraphs in infinite complete graphs, Mathematica Pannonica 1 (1990), 5-13.

[7] P. Erdős and Zs. Tuza, Rainbow subgraphs in edge-colorings of complete graphs, Ann. Discrete Math. 55 (1993), 81-88.

[8] A.M. Frieze and B.A. Reed, Polychromatic Hamilton cycles, Discrete Math. 118 (1993), 69-74.

[9] G. Hahn and C. Thomassen, Path and cycle sub-Ramsey numbers and edge-coloring conjecture, Discrete Math. 62(1) (1986), 29-33.

[10] Y. Manoussakis, M. Spyratos and Zs. Tuza, Cycles of given color patterns, J. Graph Theory 21 (1996), 153-162.

[11] Y. Manoussakis, M. Spyratos, Zs. Tuza and M. Voigt, Minimal colorings for properly colored subgraphs, Graphs and Combin. 12 (1996), 345-360. 